\title{
Special geometry of Euclidean supersymmetry IV: the local c-map
}

\author{
V. Cortés, ${ }^{a}$ P. Dempster, ${ }^{b}$ T. Mohaupt ${ }^{c}$ and O. Vaughan ${ }^{a}$ \\ ${ }^{a}$ Department of Mathematics and Center for Mathematical Physics, University of Hamburg, \\ Bundesstraße 55, D-20146 Hamburg, Germany \\ ${ }^{b}$ School of Physics $\&$ Astronomy and Center for Theoretical Physics, Seoul National University, \\ Seoul 151-747, Korea \\ ${ }^{c}$ Department of Mathematical Sciences, University of Liverpool, \\ Peach Street, Liverpool L69 7ZL, U.K. \\ E-mail: vicente.cortes@math.uni-hamburg.de, pdemp@snu.ac.kr, \\ thomas.mohaupt@liv.ac.uk, owen.vaughan@math.uni-hamburg.de
}

ABSTRACT: We consider timelike and spacelike reductions of $4 \mathrm{D}, \mathcal{N}=2$ Minkowskian and Euclidean vector multiplets coupled to supergravity and the maps induced on the scalar geometry. In particular, we investigate (i) the (standard) spatial c-map, (ii) the temporal $c$-map, which corresponds to the reduction of the Minkowskian theory over time, and (iii) the Euclidean c-map, which corresponds to the reduction of the Euclidean theory over space. In the last two cases we prove that the target manifold is para-quaternionic Kähler.

In cases (i) and (ii) we construct two integrable complex structures on the target manifold, one of which belongs to the quaternionic and para-quaternionic structure, respectively. In case (iii) we construct two integrable para-complex structures, one of which belongs to the para-quaternionic structure.

In addition we provide a new global construction of the spatial, temporal and Euclidean $c$-maps, and separately consider a description of the target manifold as a fibre bundle over a projective special Kähler or para-Kähler base.

KEYwords: Differential and Algebraic Geometry, Supergravity Models

ARXiv EPrint: 1507.04620 


\section{Contents}

1 Introduction and summary of results $\quad 2$

1.1 Background and motivation 2

1.2 Main results 6

$2 \varepsilon$-Kähler and $\varepsilon$-quaternionic Kähler geometry 11

$2.1 \varepsilon$-Kähler manifolds 11

2.1.1 $\varepsilon$-complex vector spaces 11

2.1.2 Spaces of $\varepsilon$-complex lines 12

$\begin{array}{lll}2.1 .3 & \text { Representation as symmetric spaces } & 14\end{array}$

$\begin{array}{lll}2.1 .4 & \text { Realisation as a solvable Lie group } & 16\end{array}$

$\begin{array}{lll}2.2 & \text { Special } \varepsilon \text {-Kähler manifolds } & 19\end{array}$

$2.3 \varepsilon$-quaternionic Kähler manifolds 22

2.3.1 $\varepsilon$-quaternionic structure on a vector space 22

2.3.2 The $H \otimes E$ formalism 23

2.3.3 $\varepsilon$-quaternionic structure on the tangent bundle 24

3 Dimensional reduction of four-dimensional vector multiplets $\quad \mathbf{2 6}$

$\begin{array}{lll}3.1 & \text { Four-dimensional vector multiplets } & 26\end{array}$

$\begin{array}{lll}3.2 & \text { Reduction to three dimensions } & 28\end{array}$

4 Global construction of the $c$-map $\quad 30$

4.1 Geometric data on a conic affine special $\varepsilon$-Kähler manifold 30

$\begin{array}{ll}\text { 4.2 The } c \text {-map for various spacetime signatures } & 35\end{array}$

4.2.1 A co-frame of $P$ adapted to the pull back of the $c$-map metric $\quad 37$

$\begin{array}{lll}4.2 .2 & \text { The } \varepsilon \text {-quaternionic structure } & 38\end{array}$

$5 \quad$ Levi-Civita connection and integrable $\varepsilon$-complex structures $\quad 40$

5.1 Calculating on $P$ using a non-invariant partial co-frame 41

5.2 Alternative approach: calculating on $\bar{N}$ using a co-frame 43

5.3 The spatial $c$-map 43

5.4 The temporal $c$-map $\quad 47$

$\begin{array}{ll}\text { 5.5 The Euclidean } c \text {-map } & 48\end{array}$

6 c-map spaces as fibre bundles with bundle metrics 50

6.1 The bundle metric 50

6.2 Kähler potentials for the fibre metrics 53 


\section{Introduction and summary of results}

\subsection{Background and motivation}

This paper completes the programme started in [1] and continued in [2,3], the purpose of which is to describe the scalar geometries of Euclidean $\mathcal{N}=2$ vector and hypermultiplets both without and with coupling to supergravity. Recall that with the standard (Minkowskian) spacetime signature the scalar manifolds of four-dimensional vector multiplets are affine special Kähler in the absence of supergravity and projective special Kähler in the presence of supergravity [4-13]. The scalar manifolds of hypermultiplets in $d \leq 6$ space-time dimensions are hyper-Kähler in the absence of supergravity and quaternionic Kähler in the presence of it [14-16]. Together with the affine and projective special real target manifolds of five-dimensional vector multiplets $[17,18]$, they form a family of related geometries which we refer to as special geometries. ${ }^{1}$ In each case the corresponding special geometry exists in a 'rigid' or 'affine' version, which is realised in supersymmetric field theories not coupled to supergravity, and a 'local' or 'projective' version, which occurs when the respective matter supermultiplet is coupled to supergravity. When constructing supergravity theories using the so-called conformal calculus, see [21] for a review, it is manifest that the 'local' versions of the special geometries are related to special cases of their 'global' counterparts. In the field theoretic framework, one starts with a field theory invariant under rigid superconformal transformations, and then gauges the superconformal symmetry to obtain a theory which is 'gauge-equivalent' to a Poincaré supergravity theory. The scalar geometries of the superconformal and of the Poincaré supergravity theory are related by a so-called superconformal quotient. Geometrically, the target manifolds of superconformal field theories admit a certain homothetic action of the group $\mathbb{R}^{>0}, \mathbb{C}^{*}$ and $\mathbb{H}^{*} / \mathbb{Z}_{2}$ for five-dimensional vector multiplets, four-dimensional vector multiplets, and hypermultiplets, respectively. We refer to such affine special manifolds as conical, since their metrics have the form of a metric cone, at least locally. The corresponding 'local' special geometry is then obtained by dividing out this group action. This motivates the terminology of 'conic (affine)' and 'projective' special geometry, which was introduced in [13] and [12], respectively, and which we will use in the following.

Another link between the special geometries is provided by dimensional reduction. Reducing five-dimensional vector multiplets to four-dimensional vector multiplets and fourdimensional vector multiplets to three-dimensional hypermultiplets induces maps between their scalar manifolds. These come in both a rigid and local (or supergravity) version, depending on whether the theory is coupled to supergravity. The (rigid/supergravity) $r$-map relates (affine/projective) special real to (affine/projective) special Kähler geometry [1, 22, 23], while the (rigid/supergravity) c-map relates special Kähler geometry to hyper-Kähler or quaternionic Kähler geometry [24, 25].

Throughout the programme [1-3] we have taken the approach of obtaining the scalar geometries of the Euclidean theories by dimensional reduction of Minkowskian theories over time, since this automatically ensures that the reduced theory is invariant under

\footnotetext{
${ }^{1}$ See also $[11,19-21]$ for reviews of special geometry.
} 
the Euclidean supersymmetry algebra. Thus our programme amounts to constructing and studying new versions of the $r$-map and $c$-map. It is well known that the spatial and temporal reduction of a given theory differ by relative signs in their Lagrangians, and in particular that temporal reduction can lead to scalar target spaces with indefinite Riemannian metrics. The central observation of [1] was that the scalar geometries of Minkowskian and Euclidean vector multiplets of the same dimension are related systematically by replacing complex structures by para-complex structures. ${ }^{2}$ This is in contrast with four-dimensional Minkowskian and Euclidean hypermultiplets, which have the same target manifolds at least in the local case [27]. The scalar geometries of four-dimensional Euclidean vector multiplets are affine special para-Kähler in the rigid case and projective special para-Kähler in the local case, as shown in [1] and [3]. While para-Kähler manifolds had been defined previously in the mathematical literature [28, 29] (we refer to [30] for a review of the history of para-complex geometry and further references), the two types of special para-Kähler geometry were described for the first time in these references. As explained in [1], the natural expectation is that after the dimensional reduction of four-dimensional vector multiplets over time the geometry of the resulting three-dimensional Euclidean hypermultiplets is para-hyper-Kähler in the rigid case and para-quaternionic Kähler in the local case. While rigid hypermultiplets were dealt with in [2], it remains to consider local hypermultiplets in order to complete the programme.

As in the corresponding rigid case [2], we will obtain in this paper two new supergravity $c$-maps, since we can either reduce the Minkowskian theory over time, or the Euclidean theory (which was constructed in [3]) over space. We will refer to these constructions as the temporal c-map and the Euclidean c-map, respectively. Moreover, we will also revisit the standard, 'spatial', $c$-map and thus consider all possible spacelike and timelike reductions of both Minkowskian and Euclidean four-dimensional vector multiplets coupled to supergravity. The reason is that as a further main result we obtain a new global construction of the supergravity $c$-map, which we present in a uniform way for all three cases.

The $c$-map was first described in the context of the T-duality between compactifications of type-IIA and type-IIB string theories with $\mathcal{N}=2$ supersymmetry [24]. Upon reduction to three dimensions as an intermediate step, four-dimensional vector multiplets become three-dimensional hypermultiplets, so that the three-dimensional theories have two hypermultiplet sectors which only couple gravitationally. As a result there are two different decompactification limits, which can be used to relate the four-dimensional IIA and IIB theories to one another. The hypermultiplet metrics resulting from dimensional reduction were described explicitly in [25], and it was shown that they are quaternionic Kähler, as predicted by supersymmetry. In the construction of [25] it is assumed that the underlying projective special Kähler manifold $\bar{M}$ is a projective special Kähler domain, that is defined by a single holomorphic prepotential, which is sufficient to obtain a local description of the resulting quaternionic Kähler manifold $\bar{N}$. This leaves open the question of how to describe the $c$-map globally if $\bar{M}$ is not a domain, and how to characterise the resulting quaternionic Kähler metric globally in terms of the geometric data of $\bar{M}$. A global description is not

\footnotetext{
${ }^{2}$ Related observations were already made for ten-dimensional IIB supergravity in [26].
} 
only preferable mathematically but also needed for physical questions. In particular, in order to understand the full non-perturbative dynamics of $\mathcal{N}=2$ string compactifications, one would like to know under which conditions the resulting hypermultiplet manifolds are complete. Some results on these global questions will be discussed below.

For the rigid $r$-map and $c$-map the global geometrical description is known. It was already observed in [24] that the image of an affine special Kähler domain $M$ under the rigid $c$-map can be interpreted as its cotangent bundle $T^{*} M$. More generally, affine special real and affine special Kähler manifolds are by definition equipped with a flat connection, which allows their tangent bundle to be decomposed into a horizontal and a vertical distribution. This can be used to show that the tangent bundle (equivalently, the cotangent bundle) of an affine special real or affine special Kähler manifold naturally carries the structure of an affine special (para-)Kähler or of a (para-)hyper-Kähler manifold, respectively [3, 13, 23].

Given that the affine and projective special geometries are related by superconformal quotients, one may ask whether it is possible to express the supergravity $c$-map in terms of the rigid $c$-map, applied to the associated conical affine special Kähler manifold. In physical terms this amounts to 'lifting the supergravity $c$-map to the superconformal level', which was investigated in [31] and [32]. Both constructions give rise to an off-shell realisation of the $c$-map in terms of tensor multiplets. Being off-shell means that supersymmetry is realised independently of the equations of motion by the inclusion of auxiliary fields. This has in particular the advantage that the problem of adding higher derivative terms is tractable. Tensor multiplets are related to hypermultiplets by a duality transformation. The corresponding relation between the Kähler and quaternionic Kähler metrics is as follows: the potential for the tensor multiplet metric is related to the prepotential of the special Kähler metric by a contour integral. Performing a Legendre transform on the tensor multiplet potential one obtains a hyper-Kähler potential for the hyper-Kähler cone (or Swann bundle) over the quaternionic Kähler manifold, which encodes the quaternionic Kähler metric [31].

Another approach to relating the supergravity $c$-map to the rigid $c$-map, and similarly, the supergravity $r$-map to the rigid $r$-map was described in [33]. Here the idea is to find a construction, dubbed 'conification', which allows one to obtain the image of the supergravity $c$-map (supergravity $r$-map) by conification of the image of the rigid $c$-map (or $r$-map) followed by a superconformal quotient. A general construction for the conification of Kähler manifolds and hyper-Kähler manifolds (satisfying certain technical conditions) was given. While the conification of (pseudo-)Kähler manifolds leads to a new Kähler/Kähler ('K/K') correspondence, the conification of (pseudo-)hyper-Kähler manifolds leads to a general (indefinite) version of the hyper-Kähler/quaternionic Kähler ('HK/QK') correspondence of [34], which was also discussed by [35] and [36-39]. Moreover one obtains a new explicit expression for the quaternionic Kähler metric, which allows one to recover the explicit form of the $c$-map metric of [25] and its one-loop deformation [40] as a special case, see $[33,41]$ for details. This method provides a direct proof that these metrics are quaternionic Kähler, which is independent of supersymmetry or the proofs in the undeformed case given in $[25,42]$. As a consequence, one recovers the earlier result of [35], obtained using twistor methods, that applying the QK/HK correspondence 
(inverse to the HK/QK-correspondence) to the Ferrara-Sabharwal metric one obtains the rigid $c$-map metric.

We remark that since every (para-)quaternionic Kähler manifold has an associated twistor or para-twistor space [43,44], one can also approach the geometry of the c-map through the corresponding twistor spaces. For this approach we refer to the literature, see in particular [43].

Another approach to the global description of the $c$-map is to cover the initial projective special Kähler manifold by projective special Kähler domains, to which one applies the supergravity $c$-map as formulated in [25], and then to check that the resulting quaternionic Kähler domains can be consistently glued to a quaternionic Kähler manifold. It was shown in [45] that the quaternionic Kähler domains take the form $\bar{N}=\bar{M} \times G$, where $G$ is a solvable Lie group, and that the quaternionic Kähler metric is a bundle metric $g_{\bar{N}}=g_{\bar{M}}+g_{G}(p)$, where $g_{G}(p)$ is a family of left-invariant metrics on $G$ parametrised by $p \in \bar{M} .^{3}$ This was used to prove that the quaternionic Kähler domains obtained by applying the supergravity $c$-map domain-wise can be glued together such that resulting manifold has a well-defined quaternionic Kähler structure. Moreover, it was proved in [45], that both the supergravity $r$-map and the supergravity $c$-map preserve completeness of the Riemannian metrics. While complete projective special real curves and surfaces were classified in [45] and [46] respectively, a necessary and sufficient condition for the completeness of a projective special real manifold was obtained more recently in [47]. In fact, it was shown that a projective special real manifold $\mathcal{H} \subset \mathbb{R}^{n+1}$ is complete if and only if it is closed as a subset of $\mathbb{R}^{n+1}$, a condition which can be easily checked in many examples. Moreover it was shown that any projective special real manifold respecting a generic regularity condition on its boundary is complete. Therefore the composed $r$ - and $c$-map can be used to construct many new examples of non-homogeneous complete quaternionic Kähler manifolds.

Yet another description of the spatial and temporal $c$-map was obtained in [48], where the objective was to find a formulation of the temporal $c$-map which is adapted to lifting three-dimensional Euclidean supergravity solutions ('instantons') to four-dimensional stationary supergravity solutions (black holes and other 'solitons') [48-50]. To maintain the symplectic covariance of the four-dimensional theory, dimensional reduction was performed without taking the superconformal quotient in the four-dimensional theory, which resulted in the description of the (para-)quaternionic Kähler manifold $\bar{N}$ in terms of a U(1) principal bundle $P \rightarrow \bar{N}$. In this paper we will extend the local description given in [48] to the Euclidean $c$-map. Moreover, we will give a global construction of the bundle $P$ and show that it is obtained with all data needed to define the (para-)quaternionic structure of $\bar{N}$ in a natural way from the underlying projective special (para-)Kähler manifold $\bar{M}$ as a one-dimensional extension of the tangent bundle $T M$ of the associated conical affine special (para-)Kähler manifold $M$. Another approach, left to the future, would be to adapt the HK/QK-correspondence to encompass para geometries.

One ingredient of [48] which will be useful in the present paper is to employ special real coordinates for the conical special (para-)Kähler manifold $M$. Special real coordinates

\footnotetext{
${ }^{3}$ It was already observed in [25] that by fixing a point $p \in \bar{M}$ one obtains a Kähler metric.
} 
make explicit the flat symplectic (rather then holomorphic) aspects of special Kähler manifolds $[12,13,24,51]$. From the affine point of view the existence of special real coordinates is related to the fact that any simply connected affine special Kähler manifold can be realised as a parabolic affine hypersphere [52], while the natural $S^{1}$ bundle over the associated projective special Kähler manifold carries the structure of a proper affine hypersphere endowed with a Sasakian structure [53]. Analogously, affine special para-Kähler manifolds are intrinsically improper affine hyperspheres [54]. Real coordinates play a central role in the analysis of black hole partition functions and their relation to the topological string [55-58]. The formalism of $[48,56]$ uses special real coordinates on a conical affine special Kähler manifold to describe the underlying projective special Kähler manifold. A different approach where special real coordinates are introduced directly on the projective special Kähler manifold was described in [59] (see also [60] for a review of special real coordinates in the affine case).

One aim of our programme is to make explicit the fact that Minkowskian and Euclidean theories can be presented in a uniform way. In [1] it was noted that in suitable coordinates the Lagrangian and supersymmetry transformations of vector multiplets take exactly the same form in either signature, and are only distinguished by interpreting the involution $z \mapsto \bar{z}$ as complex conjugation in the Minkowskian and as para-complex conjugation in the Euclidean case. Starting from [3] a unified $\varepsilon$-complex notation was used, where $\varepsilon=-1$ corresponds to the complex and $\varepsilon=1$ to the para-complex case. This notation will also be used in the present paper. Since, apart from choosing to reduce a Minkowskian theory over space or over time, we can choose to start with a Euclidean theory in four dimensions, we will need a further refinement of our notation. Our convention is that whenever we talk about complex/para-complex manifolds or structures in a generic way, we will use the symbol $\varepsilon= \pm 1$, whereas $\epsilon_{1}= \pm 1$ refers to the geometry of the four-dimensional theory we start with, while $\epsilon_{2}= \pm 1$ distinguishes between reduction over time and reduction over space. We will explain more about this notation in the next subsection.

The temporal $c$-map has been studied before in various publications, mostly in relation to constructing stationary solutions by lifting Euclidean solutions over time. In [61] a list of the symmetric spaces resulting from applying the temporal $c$-map to symmetric projective special Kähler manifolds was given. As mentioned in [61], these symmetric spaces are indeed para-quaternionic Kähler. This can be proved by either analysing the holonomy representation, or by comparing with the classification of pseudo-Riemannian symmetric para-quaternionic Kähler manifolds of [62]. ${ }^{4}$

\subsection{Main results}

Recall that given a projective special Kähler domain $\bar{M}$ of dimension $2 n$, defined by a holomorphic prepotential $F$ that is homogeneous of degree two, the supergravity $c$-map assigns a quaternionic Kähler metric $g_{\bar{N}}$ on a manifold $\bar{N}$ of dimension $4 n+4$. The target metric is induced by the dimensional reduction of $4 \mathrm{D}, \mathcal{N}=2$ supergravity coupled to $n$ vector multiplets over a spacelike dimension, and was first computed explicitly by Ferrara

\footnotetext{
${ }^{4}$ See also section 3.6 of [63].
} 
and Sabharwal in [25]. Henceforth we shall refer to this construction specifically as the spatial c-map. It turns out that this metric can be defined even if the projective special Kähler manifold is not defined by a single holomorphic prepotential, but is rather covered by domains on which such prepotentials exist [45]. The total space $\bar{N}$ is then interpreted as a bundle over $\bar{M}$, the fibres of which are solvable Lie groups isomorphic to the Iwasawa subgroup of $\mathrm{SU}(1, n+2)$.

The main purpose of this paper is to generalise the spatial $c$-map construction. We will give a different description of the total space $\bar{N}$ as an $S^{1}$-quotient $\bar{N}=P / S^{1}$, where $P=T M \times \mathbb{R}$ is the product of the tangent bundle of the $(2 n+2)$-dimensional conical affine special Kähler manifold $(M, J, g, \nabla, \xi)$ underlying $\bar{M}$ with the real line. We will assume that $M$ is simply connected in which case one may identify $T M=M \times \mathbb{R}^{2 n+2}$ using the flat connection and $P=M \times \mathbb{R}^{2 n+3}$. The principal $S^{1}$-action on $P$ corresponds to the $\mathrm{U}(1)$ subgroup of the natural $\mathbb{C}^{*}$-action on the first factor. It is locally generated by the trivial extension $Z_{P}$ to $P$ of the Killing vector field $J \xi$ on $M$. An advantage of this construction is that it does not place any restrictions on the projective special Kähler manifold $\bar{M}$, only that the underlying conic affine special Kähler manifold $M$ is simply connected. It can also be adapted to the following two new cases, which is the main goal of this paper:

(i) The temporal c-map.

This assigns to every projective special Kähler manifold of dimension $2 n$ a paraquaternionic Kähler manifold of dimension $4 n+4$. It is induced by the reduction of $4 D, \mathcal{N}=2$ supergravity coupled to vector multiplets over a timelike dimension.

(ii) The Euclidean c-map.

This assigns to every projective special para-Kähler manifold of dimension $2 n$ a paraquaternionic Kähler manifold of dimension $4 n+4$. It is induced by the reduction of $4 D, \mathcal{N}=2$ Euclidean supergravity coupled to vector multiplets over a spacelike dimension.

This information is summarised in table 1. While the explicit form of the target metric of the temporal and Euclidean $c$-maps can be easily adapted from the case of the spatial $c$-map, it is not obvious that the metrics are para-quaternionic Kähler. In order to prove this we will explicitly compute the Levi-Civita connection and show that it is compatible with an $\operatorname{Sp}(2) \cdot \operatorname{Sp}(2 n, \mathbb{R})$-structure. We will see that the reduced scalar curvature for all $c$-map target manifolds ${ }^{5}$ is equal to -2 .

It was observed in [64] that the target manifold of the spatial $c$-map admits a complex structure which is part of the quaternionic Kähler structure. We will show that it also admits a second complex structure which is not part of the quaternionic Kähler structure. Similarly, the temporal $c$-map admits two complex structures, one of which is part of the para-quaternionic Kähler structure, and the Euclidean c-map admits two para-complex structures, one of which is part of the para-quaternionic Kähler structure.

Let us give a brief summary of our construction for the spatial $c$-map. In order to define the quaternionic Kähler metric we must first recall some facts concerning conical

\footnotetext{
${ }^{5}$ In the conventions of [25] the reduced scalar curvature is -1 for the spatial $c$-map.
} 


\begin{tabular}{|c|c|c|c|}
\hline & Base & Target & Spacetime signature \\
\hline spatial & projective special & quaternionic & $(3+1) \rightarrow(2+1)$ \\
$c$-map & Kähler & Kähler & \\
\hline temporal & projective special & para-quaternionic & $(3+1) \rightarrow(3+0)$ \\
$c$-map & Kähler & Kähler & \\
\hline Euclidean & projective special & para-quaternionic & $(4+0) \rightarrow(3+0)$ \\
$c$-map & para-Kähler & Kähler & \\
\hline
\end{tabular}

Table 1. Summary of spatial, temporal and Euclidean $c$-maps. For a base manifold of dimension $2 n$ the target manifold has dimension $4 n+4$.

affine special Kähler manifolds that can be found in $[13,48]$. Let $(M, J, g, \nabla, \xi)$ be a conic affine special Kähler manifold of complex dimension $n+1$. We will assume that $M$ is simply connected and therefore there exists a conic holomorphic nondegenerate Lagrangian immersion $\phi: M \rightarrow T^{*} \mathbb{C}^{n+1}$ that is unique up to symplectic transformations. On $M$ there exist $2 n+2$ globally-defined real functions $\left(x^{0}=\operatorname{Re} Z^{0}, \ldots, x^{n}=\operatorname{Re} Z^{n}, y_{0}=\operatorname{Re} W_{0}, \ldots, y_{n}=\right.$ Re $\left.W_{n}\right)$, where $\left(Z^{I}, W_{I}\right)_{I=0, \ldots, n}$ are complex linear coordinates on $T^{*} \mathbb{C}^{n+1}$, that satisfy $\omega=g(\cdot, J \cdot)=2 d x^{I} \wedge d y_{I}$ and locally form a $\nabla$-affine coordinate system about any point of $M$ [13, Thm 9]. Since the functions $\left(q^{a}\right)_{a=0, \ldots, 2 n+1}:=\left(x^{I}, y_{I}\right)_{I=0, \ldots, n}$ are unique up to linear symplectic transformations one may uniquely define the following global one-forms on $T M$ :

$$
q^{a} \Omega_{a b} d q^{b}, \quad \hat{q}^{a} \Omega_{a b} d q^{b}, \quad q^{a} \Omega_{a b} d \hat{q}^{b}, \quad \hat{q}^{a} \Omega_{a b} d \hat{q}^{b},
$$

where

$$
\left(\Omega_{a b}\right)=\left(\begin{array}{cc}
0 & \mathbb{1} \\
-\mathbb{1} & 0
\end{array}\right)
$$

is two times the Gram matrix $\omega\left(\frac{\partial}{\partial q^{a}}, \frac{\partial}{\partial q^{b}}\right)$ of $\omega$, i.e. $\omega=\Omega_{a b} d q^{a} \wedge d q^{b}$, and $\left(q^{a}, \hat{q}^{a}\right)$ are global functions $T M$ associated with the functions $\left(q^{a}\right)$ on $M$. The special Kähler metric $g$ on $M$ is given by the Hessian of the function $H=\frac{1}{2} g(\xi, \xi)<0$ :

$$
g=\nabla d H=H_{a b} d q^{a} d q^{b}
$$

where $H_{a b}=\frac{\partial^{2} H}{\partial q^{a} \partial q^{b}}$. The function $H$ is homogeneous of degree two with respect to the functions $\left(q^{a}\right)$. It is called the Hesse potential and, in the real formulation of special Kähler geometry, plays a role analogous to the holomorphic prepotential. The projective special Kähler metric $\bar{g}$ is related to $H$ by [48]

$$
h=h_{a b} d q^{a} d q^{b}:=\pi^{*} \bar{g}=-\frac{1}{2 H} \nabla d H+\frac{1}{4 H^{2}}(d H)^{2}+(v)^{2}=\nabla d \tilde{H}-(d \tilde{H})^{2}+(v)^{2},
$$

where $\tilde{H}=-\frac{1}{2} \log (-2 H)$ and $v=-\frac{1}{H} q^{a} \Omega_{a b} d q^{b}=-\frac{1}{2 H} J^{*} d H=J^{*} d \tilde{H}$. Here we have denoted by $\pi: M \rightarrow \bar{M}=M / \mathbb{C}^{*}$ the canonical projection of the $\mathbb{C}^{*}$-action on $M$, which is locally generated generated by the vector fields $\xi$ and $J \xi$. 
We will now construct the quaternionic Kähler metric on $\bar{N}=P / S^{1}$. We first remark that the symmetric $(0,2)$-tensor field

$$
h+(d \tilde{H})^{2}=\nabla d \tilde{H}+(v)^{2},
$$

on $M$ has one-dimensional kernel $\mathbb{R} J \xi$. Using the canonical projection $T M \rightarrow M$ we may consider any covariant tensor field (such as $h, H, \ldots$ ) on $M$ as a tensor field on $T M$. Similarly, any covariant tensor field on $T M$ can be considered as a tensor field on $P$ by means of the canonical projection $P=T M \times \mathbb{R} \rightarrow T M$. In particular we will consider the one-forms on $P$

$$
\begin{aligned}
u^{1} & =-d \tilde{H}=\frac{1}{2 H} H_{a} d q^{a}, & \hat{u}^{1} & =-\frac{1}{2 H} H_{a} d \hat{q}^{a}, \\
u^{2} & =-\frac{1}{2 H}\left(d \tilde{\phi}+2 \hat{q}^{a} \Omega_{a b} d \hat{q}^{b}\right), & \hat{u}^{2} & =-\frac{1}{H} q^{a} \Omega_{a b} d \hat{q}^{b}
\end{aligned}
$$

where $\tilde{\phi}$ is the coordinate in the second factor of $P=T M \times \mathbb{R}$. Let us define on $P$ the symmetric $(0,2)$-tensor field

$$
g^{\prime}=h_{a b}\left(d q^{a} d q^{b}+d \hat{q}^{a} d \hat{q}^{b}\right)+\left(u^{1}\right)^{2}+\left(u^{2}\right)^{2}+\left(\hat{u}^{1}\right)^{2}+\left(\hat{u}^{2}\right)^{2},
$$

which has kernel $\mathbb{R} Z_{P}$ and is invariant under the circle group $S_{Z_{P}}^{1}$. It induces a pseudoRiemannian metric $g_{\bar{N}}$ on $\bar{N}=P / S^{1}$, which is positive definite if the projective special Kähler metric $\bar{g}$ is positive definite. We will verify later that this metric can be brought to the standard form of the Ferrara-Sabharwal metric, and is therefore pseudoquaternionic Kähler.

When we consider the cases of the temporal and Euclidean $c$-map we will find that the tensor field $g^{\prime}$ on $P$ differs from the case of the spatial $c$-map (1.1) only by certain sign-flips. It is convenient to introduce the parameters $\epsilon_{1}, \epsilon_{2} \in\{+1,-1\}$ which are determined by the rule

$$
\left(\epsilon_{1}, \epsilon_{2}\right)= \begin{cases}(-1,-1) & \text { spatial } c \text {-map } \\ (-1,+1) & \text { temporal } c \text {-map } \\ (+1, \pm 1) & \text { Euclidean } c \text {-map }\end{cases}
$$

When we are not specifically discussing the $c$-map we will use the symbol

$$
\varepsilon=\text { 'generic' epsilon, which can be either } \pm 1 \text {. }
$$

One may interpret the parameters $\epsilon_{1}, \epsilon_{2}$ physically as follows: the choice $\epsilon_{1}=-1$ corresponds to starting with a theory of $4 \mathrm{D}, \mathcal{N}=2$ supergravity coupled to vector multiplets with Lorentzian spacetime signature, and $\epsilon_{1}=1$ to the same theory with Euclidean spacetime signature. If $\epsilon_{1}=-1$ then $\epsilon_{2}=-1$ corresponds to the dimensional reduction of this theory over a spacelike dimension, and $\epsilon_{2}=1$ to dimensional reduction over a timelike dimension. If $\epsilon_{1}=1$ then one must necessarily reduce over a spacelike dimension, which corresponds to $\epsilon_{2}=-1$. However, as we will explain later, if one chooses instead $\epsilon_{2}=1$ then the resulting target manifold is globally isometric to the case $\epsilon_{2}=-1$, and so both 
choices are mathematically equivalent. Using this notation one may write various expressions in a unified way for all three $c$-maps. For example, the expression for $g^{\prime}$ can be written for all $c$-maps as

$$
g^{\prime}=h_{a b}\left(d q^{a} d q^{b}-\epsilon_{2} d \hat{q}^{a} d \hat{q}^{b}\right)+\left(u^{1}\right)^{2}-\epsilon_{1}\left(u^{2}\right)^{2}-\epsilon_{2}\left(\hat{u}^{1}\right)^{2}+\epsilon_{1} \epsilon_{2}\left(\hat{u}^{2}\right)^{2} .
$$

Note that when $\epsilon_{1}=1$ the tensor $h$ on $M$ is of split-signature on any subspace complementary to its kernel. It is therefore clear from the above expression that $g^{\prime}$ induces a positive definite metric on $\bar{N}$ only when the metric $\bar{g}$ on $\bar{M}$ is positive definite and $\epsilon_{1}=\epsilon_{2}=-1$. For all other choices of $\epsilon_{1}$ and $\epsilon_{2}$ it induces a metric of split-signature.

We will also discuss a complementary approach to describing $c$-map target spaces locally as the product

$$
\bar{N}=\bar{M} \times G,
$$

where $\bar{M}$ is (a domain in) the original projective special $\epsilon_{1}$-Kähler base manifold and $G$ is the Iwasawa subgroup of $\mathrm{SU}(1, n+2)$. With respect to this decomposition the metric on $\bar{N}$ can be written as

$$
g_{\bar{N}}=\bar{g}+g_{G}(p),
$$

where $\bar{g}$ is the metric on $\bar{M}$ and $g_{G}(p)$ is a family of left-invariant metrics on $G$ that depends on the point $p \in \bar{M}$. We will explicitly show that for fixed $p$ the metric $g_{G}$ is a symmetric $\epsilon_{1}$-Kähler metric of constant $\epsilon_{1}$-holomorphic sectional curvature.

This paper is organised as follows. We begin in section 2 with a review of background material. In section 2.1 we discuss $\varepsilon$-complex vector spaces, spaces of $\varepsilon$-complex lines and how these can be represented as symmetric spaces and realised as solvable Lie groups, and special $\varepsilon$-Kähler manifolds. In section 2.3 we discuss $\varepsilon$-quaternionic Kähler structures on vector spaces and $\varepsilon$-quaternionic Kähler manifolds. The physical aspects of the $c$-map construction are dealt with in section 3 . We discuss theories of $4 \mathrm{D}, \mathcal{N}=2$ supergravity coupled to vector multiplets with either Lorentzian or Euclidean spacetime signature, and the reduction of such theories to three dimensions over a spacelike or timelike circle. This provides the motivation for the choice of metric on the $c$-map target manifold. In section 4 we present our construction of the $c$-map. We provide a detailed description of the target space topology, metric and $\varepsilon$-quaternionic structure. The explicit calculation of the LeviCivita connection is postponed until section 5 , where we discuss each $c$-map on a case-bycase basis. In this section we also prove the existence of two integrable $\varepsilon$-complex structures on the $c$-map target manifold. Finally, in section 6 we discuss the complementary approach to describing $c$-map target manifolds locally as group bundles. Throughout this paper we will use the index conventions

$$
\begin{array}{rlrl}
A, B, C, \ldots & =1, \ldots, n, & m, p, q, \ldots & =1, \ldots, 2 n, \\
I, J, K, \ldots & =0, \ldots, n, \quad a, b, c, \ldots=0, \ldots, 2 n+1 .
\end{array}
$$




\section{$2 \varepsilon$-Kähler and $\varepsilon$-quaternionic Kähler geometry}

\section{$2.1 \varepsilon$-Kähler manifolds}

In this section we review $\varepsilon$-complex and $\varepsilon$-Kähler manifolds, and provide some examples which we will use later in the paper. The concepts of $\varepsilon$-complex geometry allow us to talk about complex and para-complex geometry in parallel. Intuitively, para-complex geometry differs from complex geometry by replacing the field of complex numbers $\mathbb{C}$ by the ring of para-complex numbers $C=\mathbb{R} \oplus e \mathbb{R}$, where $e$ is the para-complex imaginary unit satisfying

$$
e^{2}=1, \quad \bar{e}=-e .
$$

We assume that the reader is familiar with the definitions and the relevant properties of para-complex, para-Hermitian and para-Kähler manifolds, which can be found, for instance, in [1]. As in [3] we will use a unified $\varepsilon$-complex notation and terminology, where $\varepsilon=-1$ refers to the complex case and $\varepsilon=1$ to the para-complex case. Thus, for example, we will use the symbol $i_{\varepsilon}$ to denote the complex imaginary unit $i$ in the case $\varepsilon=-1$ and the para-complex imaginary unit $e$ in the case $\varepsilon=1$ such that

$$
i_{\varepsilon}^{2}=\varepsilon 1, \quad \bar{i}_{\varepsilon}=-i_{\varepsilon} .
$$

We denote by $\mathbb{C}_{\varepsilon}=\mathbb{R}\left[i_{\varepsilon}\right]$ the ring of $\varepsilon$-complex numbers. Similarly, an almost $\varepsilon$-complex structure $J$ on a real differentiable manifold $M$ is a field of endomorphisms of the tangent bundle $T M$ such that $J^{2}=\varepsilon \mathbb{1}$, and such that the eigendistributions of $J$ have equal rank. Our convention for the relation between the $\varepsilon$-complex structure $J$, pseudo-Riemannian metric $g$ and $\varepsilon$-Kähler form $\omega$ on an $\varepsilon$-Kähler manifold $(M, J, g)$ is

$$
\omega=\varepsilon g(J \cdot, \cdot) .
$$

Among the simplest examples of $\varepsilon$-Kähler manifolds are spaces of constant $\varepsilon$ holomorphic sectional curvature, which are always (pseudo-)Riemannian locally symmetric spaces. As we will see later $c$-map spaces are fibre bundles over special $\varepsilon$-Kähler manifolds with fibres of constant $\varepsilon$-holomorphic sectional curvature. Therefore we will now discuss these spaces in some detail.

\subsection{1 $\varepsilon$-complex vector spaces}

The construction of $\varepsilon$-Kähler metrics that we are going to present is a generalisation of the well-known Fubini-Study metric on complex projective spaces $\mathbb{C} P^{n}$. Consider the vector space $\mathbb{R}^{n+1} \oplus \mathbb{R}^{n+1}=\mathbb{R}^{2 n+2}$ with coordinates

$$
(\mathbf{x}, \mathbf{y})=\left(x^{I}, y^{J}\right), \quad I, J=0, \ldots, n .
$$

We introduce a scalar product

$$
\langle(\mathbf{x}, \mathbf{y}),(\mathbf{u}, \mathbf{v})\rangle=\eta(\mathbf{x}, \mathbf{u})-\varepsilon \eta(\mathbf{y}, \mathbf{v}),
$$


on $\mathbb{R}^{2 n+2}$, where $\eta(\cdot, \cdot)$ is a scalar product of signature $(k, \ell)$ on $\mathbb{R}^{n+1}$. In terms of orthonormal coordinates on $\mathbb{R}^{n+1}$ we have

$$
\eta(\mathbf{x}, \mathbf{u})=\eta_{I J} x^{I} u^{J},
$$

where $\left(\eta_{I J}\right)=\operatorname{diag}\left(\mathbb{1}_{k},-\mathbb{1}_{\ell}\right)$. Thus $\langle\cdot, \cdot\rangle$ is a scalar product of signature $(2 k, 2 \ell)$ if $\varepsilon=-1$ and of signature $(n+1, n+1)$ if $\varepsilon=1$.

Next, we define an $\varepsilon$-complex structure on $\mathbb{R}^{2 n+2}$ by

$$
J(\mathbf{x}, \mathbf{y})=(\varepsilon \mathbf{y}, \mathbf{x}) .
$$

Note that $J$ is skew with respect to $\langle\cdot, \cdot\rangle$, so that $\left(\mathbb{R}^{2 n+2}, J,\langle\cdot, \cdot\rangle\right)$ is an $\varepsilon$-Hermitian vector space, that is a (pseudo-)Hermitian vector space ${ }^{6}$ if $\varepsilon=-1$ and a para-Hermitian vector space if $\varepsilon=1$.

We identify $\left(\mathbb{R}^{2 n+2}, J\right)$ with the standard $\varepsilon$-complex vector space $\mathbb{C}_{\varepsilon}^{n+1}=\mathbb{R}^{n+1} \oplus$ $i_{\varepsilon} \mathbb{R}^{n+1}$ by means of the isomorphism

$$
\mathbb{C}_{\varepsilon}^{n+1} \ni \mathbf{z}=\mathbf{x}+i_{\varepsilon} \mathbf{y} \mapsto(\mathbf{x}, \mathbf{y}) \in \mathbb{R}^{2 n+2} .
$$

This identifies $J$ with the standard $\varepsilon$-complex structure on $\mathbb{C}_{\varepsilon}^{n+1}$, that is $J \mathbf{z}=i_{\varepsilon} \mathbf{z}$. On $\mathbb{C}_{\varepsilon}^{n+1}$ we consider the $\varepsilon$-Hermitian form

$$
\gamma(\mathbf{z}, \mathbf{w})=\eta_{I J} z^{I} \bar{w}^{J}
$$

which is of complex signature $(k, \ell)$ if $\varepsilon=-1$. Using the isomorphism $\mathbb{C}_{\varepsilon}^{n+1} \simeq \mathbb{R}^{2 n+2}$, we can write it as

$$
\gamma=\langle\cdot, \cdot\rangle+\varepsilon i_{\varepsilon}\langle J \cdot, \cdot\rangle
$$

In coordinates it is given by

$$
\begin{aligned}
\gamma((\mathbf{x}, \mathbf{y}),(\mathbf{u}, \mathbf{v})) & =\eta_{I J} x^{I} u^{J}-\varepsilon \eta_{I J} y^{I} v^{J}+i_{\varepsilon}\left(\eta_{I J} y^{I} u^{J}-\eta_{I J} x^{I} v^{J}\right) \\
& =\eta_{I J} z^{I} \bar{w}^{J}=\gamma(\mathbf{z}, \mathbf{w})
\end{aligned}
$$

where $\mathbf{z}=\mathbf{x}+i_{\varepsilon} \mathbf{y}, \mathbf{w}=\mathbf{u}+i_{\varepsilon} \mathbf{v}$.

\subsubsection{Spaces of $\varepsilon$-complex lines}

We continue to consider the $\varepsilon$-complex vector space $\mathbb{C}_{\varepsilon}^{n+1}=\left(\mathbb{R}^{2 n+2}, J\right)$, equipped with the $\varepsilon$-Hermitian form (2.3).

Consider the open subset

$$
\mathcal{D}=\mathcal{D}_{\varepsilon}= \begin{cases}\left\{v \in \mathbb{R}^{2 n+2} \mid\langle v, v\rangle>0\right\} & \text { if } \varepsilon=-1 \\ \left\{v \in \mathbb{R}^{2 n+2} \mid\langle v, v\rangle \neq 0\right\} & \text { if } \varepsilon=+1\end{cases}
$$

and define the space of $\varepsilon$-complex lines

$$
P(\mathcal{D})=\mathcal{D} / \sim, \text { where } v \sim v^{\prime} \Leftrightarrow(\mathbb{R}+\mathbb{R} J) v=(\mathbb{R}+\mathbb{R} J) v^{\prime} .
$$

\footnotetext{
${ }^{6}$ Since we will frequently deal with indefinite scalar products, we will usually omit the prefix 'pseudo-'.
} 
This can be viewed as taking a quotient with respect to the natural action $v \mapsto z v$ of the group of units

$$
\mathbb{C}_{\varepsilon}^{*}=\left\{z \in \mathbb{C}_{\varepsilon} \mid z \text { invertible }\right\} \subset \mathbb{C}_{\varepsilon},
$$

of the ring $\mathbb{C}_{\varepsilon}$. Since this group will play some role in the following, let us make some remarks.

Remark 1. In the complex case $\mathbb{C}_{\varepsilon}^{*}$ is the multiplicative group $\mathbb{C}^{*}$ of non-zero complex numbers, which is connected. In contrast, the para-complex numbers $z=x+$ ey that are not invertible are precisely those which are located on the light cone $x^{2}-y^{2}=0$, and the group of para-complex units $C^{*}$ has four connected components:

$$
C^{*}=C_{0}^{*} \cup e C_{0}^{*} \cup-C_{0}^{*} \cup-e C_{0}^{*},
$$

where $C_{0}^{*}$ is the connected component of unity.

Remark 2. Note that when defining $\mathcal{D}$ we have excluded not only the zero vector but all null vectors. This is done for two reasons. In fact, in the case $\varepsilon=1$ there exist non-zero singular vectors, that is vectors $v$ such that the orbit $C^{*} v$ is of lower dimension than 2. In order to obtain a free action of $C^{*}$ we therefore need to exclude such vectors. This is ensured by excluding null vectors. Another reason is that, as we will see below, in order to define the induced metric on $P(\mathcal{D})$ we will have to divide by $\langle v, v\rangle$. Finally, in the case $\varepsilon=-1$, to avoid jumping of the signature of the metric on the quotient we restrict to spacelike complex lines. The restriction to spacelike lines is no loss of generality, since we can always multiply the metric by -1 . Notice that in the case $\varepsilon=1$ multiplication by e maps spacelike to timelike vectors and vice-versa, and therefore there is no notion of spacelike (nor timelike) para-complex lines.

The group $\mathbb{C}_{\varepsilon}^{*}$ acts freely and properly on $\mathcal{D}$ by $\varepsilon$-holomorphic transformations. Therefore, $P(\mathcal{D})$ is a smooth $\varepsilon$-complex manifold and $\pi: \mathcal{D} \rightarrow P(\mathcal{D})$ is an $\varepsilon$-holomorphic $\mathbb{C}_{\varepsilon^{-}}^{*}$ principal bundle.

Using the $\varepsilon$-Hermitian form $\gamma$ on $\mathbb{C}_{\varepsilon}^{n+1}$, we define an $\varepsilon$-Hermitian form $\bar{\gamma}$ on $P(\mathcal{D})$ by

$$
\gamma^{\prime}(u, v)_{p}=\bar{\gamma}_{\pi(p)}\left(d \pi_{p} u, d \pi_{p} v\right)=\frac{\gamma(u, v) \gamma(p, p)-\gamma(u, p) \gamma(p, v)}{\gamma(p, p)^{2}}
$$

where $p \in \mathcal{D}$ and $u, v \in T_{p} \mathcal{D} \simeq \mathbb{R}^{2 n+2}$. In terms of $\varepsilon$-complex coordinates, this sesquilinear form corresponds to the following tensor field on $\mathcal{D}$ :

$$
\gamma^{\prime}=\frac{\left(\eta_{K L} z^{K} \bar{z}^{L}\right) \eta_{I J}-(\eta \bar{z})_{I}(\eta z)_{J}}{\left(\eta_{K L} z^{K} \bar{z}^{L}\right)^{2}} d z^{I} \otimes d \bar{z}^{J} .
$$

To see that this defines an $\varepsilon$-Hermitian metric on $P(\mathcal{D})$, we first note that $\gamma^{\prime}$ is manifestly invariant under $\mathbb{C}_{\varepsilon}^{*}$. Moreover it is easy to see that $\gamma^{\prime}(\xi, \cdot)=0=\gamma^{\prime}(J \xi, \cdot)$, where $\xi=$ $z^{I} \frac{\partial}{\partial z^{I}}+\bar{z}^{I} \frac{\partial}{\partial \bar{z}^{I}}$ is the position vector field on $\mathcal{D}$. Thus $\gamma^{\prime}$ is also horizontal with respect to the $\mathbb{C}_{\varepsilon}^{*}$-action and hence can be pushed down to $P(\mathcal{D})$. Since the kernel of $\gamma^{\prime}$ is spanned by $\xi, J \xi$, it defines a non-degenerate $\varepsilon$-Hermitian metric on $P(\mathcal{D})$. 
Consequently the real part

$$
g^{\prime}(u, v)_{p}=\frac{\langle u, v\rangle\langle p, p\rangle-\langle u, p\rangle\langle v, p\rangle+\varepsilon\langle u, J p\rangle\langle v, J p\rangle}{\langle p, p\rangle^{2}},
$$

of $\gamma^{\prime}$ defines a metric $\bar{g}$ on $P(\mathcal{D})$ such that $g^{\prime}=\pi^{*} \bar{g}$. The degenerate tensor field $g^{\prime}$ on $\mathcal{D}$, when expressed in $\varepsilon$-complex coordinates, is

$$
g^{\prime}=g_{I \bar{J}} d z^{I} d \bar{z}^{J}=\frac{1}{\left(\eta_{K L} z^{K} \bar{z}^{L}\right)^{2}}\left(\left(\eta_{K L} z^{K} \bar{z}^{L}\right) \eta_{I J}-\eta_{I K} \bar{z}^{K} \eta_{J L} z^{L}\right) d z^{I} d \bar{z}^{J} .
$$

This symmetric tensor field can be locally expressed using a potential $K$, which is given by the logarithm of the squared length of the position vector field:

$$
g_{I \bar{J}}=\frac{\partial^{2} K}{\partial z^{I} \partial \bar{z}^{J}}
$$

where

$$
K=\log \left|\eta_{K L} z^{K} \bar{z}^{L}\right|=\log |\langle\xi, \xi\rangle| .
$$

We can also describe the metric $\bar{g}$ using inhomogeneous coordinates on $P(\mathcal{D})$, instead of using the symmetric tensor field $g^{\prime}$ on $\mathcal{D}$. If we identify $P(\mathcal{D})$ locally with the hypersurface $z^{0}=1$ the associated inhomogeneous coordinates are $z^{A}, A=1, \ldots, n$. In terms of these coordinates,

$$
\bar{g}=\left(1-\bar{\eta}_{C D} z^{C} \bar{z}^{D}\right)^{-2}\left(-\left(1-\bar{\eta}_{C D} z^{C} \bar{z}^{D}\right) \bar{\eta}_{A B}-\bar{\eta}_{A C} \bar{z}^{C} \bar{\eta}_{B D} z^{D}\right) d z^{A} d \bar{z}^{B}
$$

For later convenience we have taken $\eta_{00}=1$, which will cover all cases relevant to us, and defined $\bar{\eta}_{A B}=-\eta_{A B}$. Thus $\bar{\eta}_{A B}$ has signature $(\ell, k-1)$. The tensor $\bar{g}$ is an $\varepsilon$-Kähler metric on $P(\mathcal{D})$ with $\varepsilon$-Kähler potential

$$
\bar{K}=\log \left|1-\bar{\eta}_{A B} z^{A} \bar{z}^{B}\right| .
$$

It is straightforward to check that the $\varepsilon$-Kähler metric $\bar{g}$ has constant $\varepsilon$-holomorphic sectional curvature, that is the sectional curvature of a $J$-invariant plane does not depend on the chosen plane. We will recover this later using an alternative description of these spaces (with the exception of $\mathbb{C} P^{n}$ ) in terms of open orbits of solvable Lie groups. It is known ${ }^{7}$ that $\varepsilon$-Kähler spaces with constant $\varepsilon$-holomorphic sectional curvature $c$ are locally symmetric and locally uniquely determined by the value of the constant $c$. Next we discuss in more detail the spaces $P(\mathcal{D})$ as globally symmetric $\varepsilon$-Kähler spaces, which we represent as coset spaces.

\subsubsection{Representation as symmetric spaces}

The space $P(\mathcal{D})$ is the space of $\varepsilon$-complex lines in an open subset $\mathcal{D}$ of the $\varepsilon$-complex vector space $\mathbb{C}_{\varepsilon}^{n+1}$. We will now describe it as a symmetric space. Let $G \subset G L(2 n+2, \mathbb{R})$ be the $\varepsilon$-unitary group of $\mathbb{C}_{\varepsilon}^{n+1}=\mathbb{R}^{2 n+2}$, that is the group of $\varepsilon$-complex linear transformations

\footnotetext{
${ }^{7}$ This can be proven as in the case of (positive definite) Kähler manifolds of constant holomorphic sectional curvature, see [65, Ch. IX, Thm. 7.9].
} 
which leave the scalar product $\langle\cdot, \cdot\rangle$ invariant. For $\varepsilon=-1$ this is the (pseudo-)unitary group $\mathrm{U}(k, \ell)$, while for $\varepsilon=1, G$ is the para-unitary group, which is isomorphic to $G L(n+1, \mathbb{R})[1]$. More precisely, the representation of the para-unitary group on $\mathbb{R}^{2 n+2}$ is equivalent to the sum of the standard $(n+1)$-dimensional representation of $G L(n+1, \mathbb{R})$ and its dual. Since the group $G$ acts transitively on $P(\mathcal{D})$ we can identify $P(\mathcal{D})$ with the corresponding homogeneous space

$$
P(\mathcal{D}) \simeq G / H
$$

where $H$ is the stabiliser of an $\varepsilon$-complex line. We notice that already the special (pseudo-)unitary group $\mathrm{SU}(k, \ell)$, respectively the special para-unitary group $\mathrm{SL}(n+1, \mathbb{R})$, acts transitively on $P(\mathcal{D})$. For notational convenience we prefer to work with the full $\varepsilon$-unitary group. Let us consider the possible cases in turn.

1. For $\varepsilon=-1$ and $\eta_{I J}=\delta_{I J}$ the Hermitian form is invariant under $\mathrm{U}(n+1)$ and $\bar{\eta}_{A B}=-\delta_{A B}$. The stabiliser of a complex line in $\mathbb{C}^{n+1}$ is $\mathrm{U}(1) \times \mathrm{U}(n)$. The resulting complex projective space is the symmetric space

$$
P(\mathcal{D})=\mathbb{C} P^{n} \simeq \mathrm{U}(n+1) /(\mathrm{U}(1) \times \mathrm{U}(n)),
$$

and the corresponding Kähler metric is the Fubini-Study metric:

$$
\bar{K}=\log \left(1+\delta_{A B} z^{A} \bar{z}^{B}\right), \quad \bar{g}=\frac{\left(1+\delta_{C D} z^{C} \bar{z}^{D}\right) \delta_{A B}-\bar{z}_{A} z_{B}}{\left(1+\delta_{C D} z^{C} \bar{z}^{D}\right)^{2}} d z^{A} d \bar{z}^{B}
$$

where $z_{A}=\delta_{A B} z^{B}=z^{A}$.

2. For $\varepsilon=-1$ and $\left(\eta_{I J}\right)=\operatorname{diag}\left(1,-\mathbb{1}_{n}\right)$, the Hermitian form is invariant under $\mathrm{U}(1, n)$ and $\bar{\eta}_{A B}=\delta_{A B}$. Complex lines are stabilised by $\mathrm{U}(1) \times \mathrm{U}(n)$. The resulting symmetric space is the complex hyperbolic space

$$
P(\mathcal{D})=\mathbb{C} H^{n} \simeq \mathrm{U}(1, n) /(\mathrm{U}(1) \times \mathrm{U}(n)),
$$

which is the dual, in the sense of Riemannian symmetric spaces, of $\mathbb{C} P^{n}$. We remark that both spaces are real forms of $G L(1+n, \mathbb{C}) /(G L(1, \mathbb{C}) \times G L(n, \mathbb{C}))$. The Kähler metric $\bar{g}$ defined in the previous section is negative definite and coincides with the complex hyperbolic metric up to sign:

$$
\bar{K}=\log \left(1-\delta_{A B} z^{A} \bar{z}^{B}\right), \quad \bar{g}=-\frac{\left(1-\delta_{C D} z^{C} \bar{z}^{D}\right) \delta_{A B}+\bar{z}_{A} z_{B}}{\left(1-\delta_{C D} z^{C} \bar{z}^{D}\right)^{2}} d z^{A} d \bar{z}^{B},
$$

where $z_{A}=\bar{\eta}_{A B} z^{B}=z^{A}$.

3. For $\varepsilon=-1$ and $\left(\eta_{I J}\right)=\operatorname{diag}\left(\mathbb{1}_{k},-\mathbb{1}_{\ell}\right)$, the Hermitian form is invariant under $\mathrm{U}(k, \ell)$ and lines are stabilised by $\mathrm{U}(1) \times \mathrm{U}(k-1, \ell)$ and $\left(\bar{\eta}_{A B}\right)=\operatorname{diag}\left(-\mathbb{1}_{k-1}, \mathbb{1}_{\ell}\right)$. The resulting symmetric spaces

$$
P(\mathcal{D})=\mathbb{C} H^{(k-1, \ell)} \simeq \mathrm{U}(k, \ell) /(\mathrm{U}(1) \times \mathrm{U}(k-1, \ell)),
$$


are indefinite signature versions of the Hermitian symmetric spaces $\mathbb{C} P^{n}$ and $\mathbb{C} H^{n}$. We remark that they are again real forms of $G L(1+n, \mathbb{C}) /(G L(1, \mathbb{C}) \times G L(n, \mathbb{C}))$. The resulting pseudo-Kähler metric has complex signature $(k-1, \ell)$ :

$$
\bar{K}=\log \left(1-\bar{\eta}_{A B} z^{A} \bar{z}^{B}\right), \quad \bar{g}=-\frac{\left(1-\bar{\eta}_{C D} z^{C} \bar{z}^{D}\right) \bar{\eta}_{A B}+\bar{z}_{A} z_{B}}{\left(1-\bar{\eta}_{C D} z^{C} \bar{z}^{D}\right)^{2}} d z^{A} d \bar{z}^{B}
$$

since $\left(\bar{\eta}_{A B}\right)$ has signature $(\ell, k-1)$, and where $z_{A}=\bar{\eta}_{A B} z^{B}$.

4. We finally consider the para-complex case, $\varepsilon=1$. The stabiliser of a point of $P(\mathcal{D})$ under the para-unitary group $G L(n+1, \mathbb{R})$ is $G L(1, \mathbb{R}) \times G L(n, \mathbb{R})$. The resulting space

$$
P(\mathcal{D})=C H^{n} \simeq G L(n+1, \mathbb{R}) / G L(1, \mathbb{R}) \times G L(n, \mathbb{R}),
$$

is the para-complex analogue of any of the above spaces, which for convenience is referred to as para-complex hyperbolic space. The corresponding symmetric space is yet another real form of $G L(n+1, \mathbb{C}) /(G L(1, \mathbb{C}) \times G L(n, \mathbb{C}))$. The resulting para-Kähler metric has real signature $(n, n)$ irrespective of the signature of $\left(\eta_{I J}\right)=$ $\left(1,-\left(\bar{\eta}_{A B}\right)\right)$ :

$$
\bar{K}=\log \left|1-\bar{\eta}_{A B} z^{A} \bar{z}^{B}\right|, \quad \bar{g}=-\frac{\left(1-\bar{\eta}_{C D} z^{C} \bar{z}^{D}\right) \bar{\eta}_{A B}+\bar{z}_{A} z_{B}}{\left(1-\bar{\eta}_{C D} z^{C} \bar{z}^{D}\right)^{2}} d z^{A} d \bar{z}^{B},
$$

where $z_{A}=\bar{\eta}_{A B} z^{B}$.

\subsubsection{Realisation as a solvable Lie group}

Recall that the Iwasawa subgroup $L$ of a non-compact semi-simple group $G$ is the maximal triangular (and, hence, solvable) Lie subgroup of $G$, which is unique up to conjugation. As a consequence of the Iwasawa decomposition it acts simply transitively on the corresponding Riemannian symmetric space of the non-compact type $G / H$, which is defined as the quotient of $G$ by its maximal compact subgroup $H$, which is unique up to conjugation. Standard examples include hyperbolic spaces such as $G / H=\mathrm{SU}(1, \tilde{n}+2) / S(\mathrm{U}(1) \times \mathrm{U}(\tilde{n}+2))$. This allows us to identify $G / H$ with $L$ and to compute geometric quantities on $G / H$, such as the Levi-Civita connection and curvature, purely algebraically on the Lie algebra $\mathfrak{l}$ of $L$. On pseudo-Riemannian symmetric spaces $G / H$ the group $L$, in general, no longer acts transitively, but it may still act at least with open orbit such that we can still identify the symmetric space with $L$ locally and perform computations on $\mathfrak{l}$. This is indeed the case for all non-compact symmetric spaces of constant $\varepsilon$-holomorphic sectional curvature considered in the previous subsection. In fact, in this section, we show explicitly that the Iwasawa subalgebra $\mathfrak{l} \subset \mathfrak{s u}(1, \tilde{n}+2)$ can be equipped with a scalar product $\langle\cdot, \cdot\rangle$ and $\varepsilon$-complex structure $J$, such that the metric on $L$ obtained by left-invariant extension of the scalar product is $\varepsilon$-Kähler and has constant $\varepsilon$-holomorphic sectional curvature. Depending on our choice of scalar product, this provides a local description of $\mathbb{C} H^{\tilde{n}+2}, \mathbb{C} H^{(\tilde{k}-1, \tilde{\ell})}$ where $\tilde{k}+\tilde{\ell}=\tilde{n}+3$, or $C H^{\tilde{n}+2}$ in terms of a solvable Lie group equipped with a left-invariant $\varepsilon$-Kähler metric. 
We start by reviewing the standard realisation of the Lie algebra $\mathfrak{l}$ of the Iwasawa subgroup $L \subset \mathrm{SU}(1, \tilde{n}+2)$. The $(2 \tilde{n}+4)$-dimensional Lie algebra $\mathfrak{l}$ admits the decomposition

$$
\mathfrak{l}=V \oplus \mathbb{R} Z_{0} \oplus \mathbb{R} D,
$$

where $V=\mathbb{R}^{2 \tilde{n}+2}$ is the abelian Lie algebra of dimension $2 \tilde{n}+2$. The non-trivial commutators are

$$
[X, Y]=\omega(X, Y) Z_{0}, \quad[D, X]=\frac{1}{2} X, \quad\left[D, Z_{0}\right]=Z_{0},
$$

where $X, Y \in V$, and where $\omega$ is a non-degenerate symplectic form on $V$. Thus $Z_{0}$ extends $V$ into the standard Heisenberg Lie algebra of dimension $2 \tilde{n}+3$ on which $D$ acts as a derivation. We choose an $\omega$-skew-symmetric $\varepsilon$-complex structure $J$ on $V$ which is extended to $\mathfrak{l}$ by setting

$$
J D=-Z_{0}, \quad J Z_{0}=-\varepsilon D .
$$

On $V$ this determines the (possibly indefinite) scalar product $\langle\cdot, \cdot\rangle=\omega(J \cdot, \cdot)$ which we extend orthogonally to $\operatorname{span}\left\{Z_{0}, D\right\}$ by

$$
\langle D, D\rangle=1, \quad\left\langle D, Z_{0}\right\rangle=0, \quad\left\langle Z_{0}, Z_{0}\right\rangle=-\varepsilon .
$$

This also determines the extension of the symplectic form to $\mathfrak{l}$ by $\omega\left(D, Z_{0}\right)=1$. Since the $\varepsilon$-complex structure is skew-symmetric with respect to the scalar product, $J \in \mathfrak{s o}(\mathfrak{l},\langle\cdot, \cdot\rangle)$, we can express it in terms of bivectors as

$$
J=D \wedge Z_{0}+J \circ \operatorname{pr}_{V}
$$

using the convention that

$$
(X \wedge Y)(Z)=X\langle Y, Z\rangle-Y\langle X, Z\rangle,
$$

for $X, Y, Z \in \mathfrak{l}$. Here $\operatorname{pr}_{V}$ denotes the projection onto $V$.

By identification of $\mathfrak{l}$ with the Lie algebra of left-invariant vector fields on the associated Lie group $L$, we obtain a left-invariant metric $g_{L}, \varepsilon$-complex structure $J$, and symplectic form $\omega$ on $L$. To compute the Levi-Civita connection $\nabla$ on $L$ we use the Koszul formula:

$$
\begin{aligned}
2\left\langle\nabla_{X} Y, Z\right\rangle= & X\langle Y, Z\rangle+Y\langle X, Z\rangle-Z\langle X, Y\rangle \\
& +\langle[X, Y], Z\rangle-\langle X,[Y, Z]\rangle-\langle Y,[X, Z]\rangle,
\end{aligned}
$$

where $X, Y, Z$ are vector fields on $L$. It is sufficient to evaluate for left-invariant vector fields, in which case the first three terms on the right hand side are zero. The remaining terms can be evaluated using the scalar product and commutator relations of $\mathfrak{l}$.

The result can be summarised as follows:

$$
\begin{aligned}
\nabla_{D} & =0 \\
\nabla_{Z_{0}} & =D \wedge Z_{0}+\frac{1}{2} J \circ \operatorname{pr}_{V}, \\
\nabla_{X} & =\frac{1}{2} D \wedge X+\frac{\varepsilon}{2} Z_{0} \wedge J X, \quad \forall X \in V .
\end{aligned}
$$

Here $\nabla_{X}$, with $X \in \mathfrak{l}$, is interpreted as an endomorphism of $\mathfrak{l}$. 
It is straightforward to verify that

$$
\nabla_{X}(J Y)=J \nabla_{X} Y \quad \forall X, Y \in \mathfrak{l} .
$$

Thus the $\varepsilon$-complex structure is parallel, $\nabla J=0$, and in particular integrable. We conclude that the metric $g_{L}$ on $L$ is a left-invariant $\varepsilon$-Kähler metric.

The curvature of the connection $\nabla$ is computed by the formula

$$
R(X, Y)=\left[\nabla_{X}, \nabla_{Y}\right]-\nabla_{[X, Y]} .
$$

$R(X, Y)$ can be interpreted as a skew endomorphism of $\mathfrak{l}$, and thus be computed on $\mathfrak{l}$. When evaluating commutators of skew endomorphisms, the following formula is useful:

$$
[X \wedge Y, Z \wedge W]=(X \wedge W)\langle Y, Z\rangle+(Y \wedge Z)\langle X, W\rangle-(X \wedge Z)\langle Y, W\rangle-(Y \wedge W)\langle X, Z\rangle .
$$

It is straightforward to show that $R(X, Y)$ takes the canonical form

$$
R(X, Y)=R(X, Y)_{\mathrm{can}}=-\frac{1}{4}(X \wedge Y-\varepsilon J X \wedge J Y+2 \omega(X, Y) J),
$$

and one easily verifies that the $\varepsilon$-holomorphic sectional curvature is -1 :

$$
\frac{\left\langle R(X, J X)_{\operatorname{can}} J X, X\right\rangle}{\langle X \wedge J X, X \wedge J X\rangle}=-1
$$

For later applications is useful to introduce a Darboux basis $\left(Y^{i}, X_{j}\right)_{i, j=0, \ldots, \tilde{n}}$ of $V$ :

$$
\omega\left(Y^{i}, X_{j}\right)=\delta_{j}^{i} \Rightarrow\left[Y^{i}, X_{j}\right]=\delta_{j}^{i} Z_{0} .
$$

The Gram matrix of the scalar product $\langle\cdot, \cdot\rangle$ on $V$ with respect to this basis is given by

$$
\left\langle Y^{i}, Y^{j}\right\rangle=\tilde{\eta}^{i j}, \quad\left\langle X_{i}, X_{j}\right\rangle=-\varepsilon \tilde{\eta}_{i j}
$$

where $\tilde{\eta}^{i j}$ has signature $(\tilde{\ell}-1, \tilde{k}-1)$, (with $\tilde{k}+\tilde{\ell}=\tilde{n}+3$, where $\left.\operatorname{dim} V=2 \tilde{n}+2\right)$ and where $\tilde{\eta}^{i k} \tilde{\eta}_{k j}=\delta_{j}^{i}$. This determines the expression of $J$ on $V$ in this basis:

$$
J Y^{i}=-\tilde{\eta}^{i j} X_{j}, \quad J X_{i}=-\varepsilon \tilde{\eta}_{i j} Y^{j} .
$$

We can choose the Darboux basis such that $\tilde{\eta}^{i j}$ is diagonal with entries \pm 1 .

Now, to finish this subsection, we would like to indicate which scalar products on $V$ correspond to which of the symmetric spaces of constant $\varepsilon$-holomorphic sectional curvature discussed in the previous subsection. At this point we anticipate some results which will be proven in section 6 . We will see in section 6 that $c$-map spaces are fibre bundles over projective special $\varepsilon$-Kähler manifolds, where the fibre is precisely the solvable Iwasawa group $L$ of $\mathrm{SU}(1, \tilde{n}+2)$ equipped with a left-invariant $\varepsilon$-Kähler metric which depends on the base point. (Here $\tilde{n}$ is the complex dimension of the base manifold.) The $c$-map will provide coordinates on $L$, which easily allow one to find the associated $\varepsilon$-Kähler potential. By comparing to the $\varepsilon$-Kähler potentials listed in section 2.1 .3 we will then be able to identify the symmetric spaces that actually occur in the context of the $c$-map. For convenience we already summarise the result here: 
1. For $\varepsilon=-1$ and $\tilde{\eta}_{i j}=\delta_{i j}$, we obtain the complex hyperbolic space $\mathbb{C} H^{\tilde{n}+2}$ equipped with the positive definite metric $-\bar{g}$.

2. For $\varepsilon=-1$ and $\tilde{\eta}_{i j}$ a matrix of signature $(\tilde{\ell}-1, \tilde{k}-1)$ we obtain the indefinite complex hyperbolic space $\mathbb{C} H^{(\tilde{\ell}, \tilde{k}-1)}$ equipped with the metric $-\bar{g}$ of complex signature $(\tilde{\ell}, \tilde{k}-1)$.

3. For $\varepsilon=1$ and any signature of $\tilde{\eta}_{i j}$ we obtain the para-complex hyperbolic space $C H^{\tilde{n}+2}$, equipped with the metric $-\bar{g}$ of real signature $(\tilde{n}+2, \tilde{n}+2)$.

Note that when choosing $\varepsilon=-1$ and a positive definite scalar product on $V$, we do not obtain a metric on the compact space $\mathbb{C} P^{\tilde{n}+2}$, but a positive definite metric on its noncompact dual $\mathbb{C} H^{\tilde{n}+2}$. We will see in section 6 why the compact space $\mathbb{C} P^{\tilde{n}+2}$ cannot arise in the context of the $c$-map.

\subsection{Special $\varepsilon$-Kähler manifolds}

For later use we now review special $\varepsilon$-Kähler manifolds, following the definitions and theorems stated in [3].

Definition 1. An affine special $\varepsilon$-Kähler (ASEK) manifold $(M, J, g, \nabla)$ is an $\varepsilon$-Kähler manifold $(M, g, J)$ endowed with a flat torsion-free connection $\nabla$ such that

1. $\nabla$ is symplectic with respect to the $\varepsilon$-Kähler form, i.e. $\nabla \omega=0$.

2. $\nabla J$ is a symmetric (1,2)-tensor field, i.e. $\left(\nabla_{X} J\right) Y=\left(\nabla_{Y} J\right) X$ for all $X, Y$.

Every simply connected $\mathrm{AS} \varepsilon \mathrm{K}$ manifold admits a canonical realisation as an immersed Lagrangian submanifold of the $\varepsilon$-complex symplectic vector space $T^{*} \mathbb{C}_{\varepsilon}^{n+1}=\mathbb{C}_{\varepsilon}^{2 n+2}$, such that the special geometry of $M$ is induced by the immersion, where $n+1$ is the $\varepsilon$-complex dimension of $M$. From this one obtains the local characterisation of an AS\&K manifold in terms of an $\varepsilon$-holomorphic prepotential. For any given $p \in M$ one can choose linear symplectic coordinates

$$
\left(X^{I}, W_{J}\right)=\left(x^{I}+i_{\varepsilon} u^{I}, y_{J}+i_{\varepsilon} v_{J}\right), \quad I, J=0, \ldots, n,
$$

in $\mathbb{C}_{\varepsilon}^{2 n+2}$ such that the symplectic form is given by $d X^{I} \wedge d W_{I}$ and the functions $X^{I}$ restrict to a system of local $\varepsilon$-holomorphic coordinates near $p$, which we call special $\varepsilon$ holomorphic coordinates. The Lagrangian submanifold is then defined by equations of the form $W_{I}=F_{I}(X):=\frac{\partial F(X)}{\partial X^{I}}$, where $F(X)=F\left(X^{0}, \ldots, X^{n}\right)$ is an $\varepsilon$-holomorphic function, which is called the prepotential. The metric is given by

$$
g=N_{I J} d X^{I} d \bar{X}^{J}, \quad N_{I J}=-i_{\varepsilon}\left(F_{I J}-\bar{F}_{I J}\right)
$$

where $F_{I J}$ are the second derivatives of the prepotential $F$, and a Kähler potential is therefore

$$
K=-i_{\varepsilon}\left(X^{I} \bar{F}_{I}-F_{I} \bar{X}^{I}\right)
$$


On $M$ the $2 n+2$ globally-defined real functions

$$
\left(q^{a}\right)=\left(x^{I}, y_{J}\right), \quad a=0, \ldots, 2 n+1,
$$

form a local system of $\nabla$-affine coordinates about any point, which we call special real coordinates. Both special $\varepsilon$-holomorphic and special real coordinates are useful when investigating $\mathrm{AS} \varepsilon \mathrm{K}$ geometry, although many of the new results in this paper will be presented in terms of the latter. The Kähler form and metric are given by the following globally-defined expressions

$$
\begin{array}{ll}
\omega=\Omega_{a b} d q^{a} \wedge d q^{b}=2 d x^{I} \wedge d y_{I}, & \left(\Omega_{a b}\right)=\left(\begin{array}{cc}
0 & \mathbb{1}_{n+1} \\
-\mathbb{1}_{n+1} & 0
\end{array}\right), \\
g=H_{a b} d q^{a} \otimes d q^{b}=H_{a b} d q^{a} d q^{b}, & H_{a b}=\frac{\partial^{2}}{\partial q^{a} \partial q^{b}} H,
\end{array}
$$

where $H$ is a globally-defined real function called the Hesse potential.

The $\varepsilon$-complex structure is determined by the metric and Kähler form according to $(2.1)$

$$
J=-\frac{1}{2} \Omega^{a c} H_{c b} \frac{\partial}{\partial q^{a}} \otimes d q^{b}
$$

and $J^{2}=\varepsilon I d$ ensures that

$$
H_{a b} \Omega^{b c} H_{c d}=\varepsilon 4 \Omega_{a d} .
$$

The matrix $\left(H_{a b}\right)$ is related to $\left(F_{I J}\right)$ by

$$
\left(H_{a b}\right)=\left(\begin{array}{cc}
N-\varepsilon R N^{-1} R & \varepsilon 2 R N^{-1} \\
\varepsilon 2 N^{-1} R & -\varepsilon 4 N^{-1}
\end{array}\right),
$$

where

$$
F_{I J}=: \frac{1}{2}\left(R_{I J}-\varepsilon i_{\varepsilon} N_{I J}\right) .
$$

The imaginary part of the $\varepsilon$-holomorphic prepotential is related to the Hesse potential by a Legendre transform $H(x, y)=-\varepsilon\left(2 \operatorname{Im} F(X(x, y))-2 y_{I} u^{I}(x, y)\right)$, which replaces the $u^{I}$ with $y_{I}$ as independent functions [66].

Definition 2. A conic affine special $\varepsilon$-Kähler (CASEK) manifold $(M, J, g, \nabla, \xi)$ is an $A S \varepsilon K$ manifold $(M, J, g, \nabla)$ equipped with a vector field $\xi$ such that

$$
\nabla \xi=D \xi=I d,
$$

where $D$ is the Levi-Civita connection.

The definition implies that $L_{\xi} g=2 g$ and $L_{J \xi} g=0$, so that while $\xi$ acts homothetically, $J \xi$ acts isometrically. Moreover the vector field $\xi$ and, hence, $J \xi$ preserves $J$ and the two vector fields generate an infinitesimal action of a two-dimensional abelian Lie algebra. The corresponding condition on the Hesse potential for an $\mathrm{AS} \varepsilon \mathrm{K}$ manifold to be conical is that it must be homogeneous of degree two, once we have restricted the special real coordinates such that $\xi$ is the corresponding Euler field, $\xi=q^{a} \frac{\partial}{\partial q^{a}}$. Such special coordinates are called conical, and it is understood in the following that special coordinates are conical. 
As in [3] we will always assume that $g(\xi, \xi)=2 H$ does not vanish on $M$, which will be used in (2.12). In addition, we will assume for simplicity that $M$ is simply connected and impose the following regularity assumption on $\mathrm{CAS} \varepsilon \mathrm{K}$ manifolds in order to discuss projective special $\varepsilon$-Kähler manifolds in a convenient way. We assume that the infinitesimal action generated by $\xi$ and $J \xi$ is induced by a principal $\mathbb{C}_{\varepsilon}^{*}$-action on $M$ and that the Lagrangian immersion $M \rightarrow \mathbb{C}_{\varepsilon}^{2 n+2}$ is $\mathbb{C}_{\varepsilon}^{*}$-equivariant.

Consider the (0,2)-tensor field

$$
h=-\frac{g}{g(\xi, \xi)}+\frac{g(\cdot, \xi) \otimes g(\cdot, \xi)-\varepsilon g(\cdot, J \xi) \otimes g(\cdot, J \xi)}{g(\xi, \xi)^{2}}
$$

on a regular $\mathrm{CAS} \varepsilon \mathrm{K}$ manifold, which is $\mathbb{C}_{\varepsilon}^{*}$-invariant and degenerate along the orbits of the $\mathbb{C}_{\varepsilon}^{*}$-action. In terms of special $\varepsilon$-complex coordinates $h$ is given by

$$
g_{I J} d X^{I} d \bar{X}^{J}=\left(-\frac{N_{I J}}{X N \bar{X}}+\frac{(N \bar{X})_{I}(N X)_{J}}{(X N \bar{X})^{2}}\right) d X^{I} d \bar{X}^{J},
$$

where $(N X)_{I}=N_{I J} X^{J}$ and $X N \bar{X}=N_{I J} X^{I} \bar{X}^{J}$, whilst in terms of special real coordinates it is given by

$$
h_{a b} d q^{a} d q^{b}=\left(-\frac{1}{2 H} H_{a b}+\frac{1}{4 H^{2}} H_{a} H_{b}-\varepsilon \frac{1}{H^{2}} \Omega_{a c} q^{c} \Omega_{b d} q^{d}\right) d q^{a} d q^{b},
$$

where

$$
\left(H_{a}\right)=\left(\frac{\partial}{\partial q^{a}} H\right)=\left(-\varepsilon 2 v_{I}, \varepsilon 2 u^{J}\right)^{T} .
$$

The requirement that a $\mathrm{CAS} \varepsilon \mathrm{K}$ manifold $M$ is regular ensures that the projection $\pi: M \rightarrow \bar{M}$ onto the space of $\mathbb{C}_{\varepsilon}^{*}$-orbits is the quotient map of a holomorphic principal bundle over an $\varepsilon$-complex manifold, and that the (0,2)-tensor field $h$ on $M$ induces an $\varepsilon$-Kähler metric $\bar{g}$ on $\bar{M}$, such that $\pi^{*} \bar{g}=h$. The $\varepsilon$-Kähler manifold $(\bar{M}, \bar{J}, \bar{g})$ is called a projective special $\varepsilon$-Kähler (PSEK) manifold. ${ }^{8}$

The following remark will be used later.

Remark 3. Note in the case $\varepsilon=1$ that the action of $i_{\varepsilon}=e \in \mathbb{C}_{\varepsilon}^{*}=C^{*}$ induces an antiisometry of the $C A S \varepsilon K$ manifold that sends $(M, J, g, \nabla, \xi)$ to $(M, J,-g, \nabla, \xi)$ but preserves the $C^{*}$-invariant tensor $h$.

The relation between a $\mathrm{CAS} \varepsilon \mathrm{K}$ manifold and the associated $\mathrm{PS} \varepsilon \mathrm{K}$ manifold is via an $\varepsilon$-Kähler quotient and generalises the Fubini-Study-type constructions of the previous section. In terms of special coordinates $\left(X^{I}\right)$ on $M$, the degenerate and $\mathbb{C}_{\varepsilon}^{*}$-invariant $(0,2)$ tensor $h$ has a potential of the form

$$
K_{h}=-\log \left|-i_{\varepsilon}\left(X^{I} \bar{F}_{I}-F_{I} \bar{X}^{I}\right)\right|,
$$

where $g_{I J}=\frac{\partial^{2} K_{h}}{\partial X^{I} \partial X^{J}}$ in the expression $(2.13)$ for $h$. One can describe $(\bar{M}, \bar{g})$ using homogeneous special $\varepsilon$-holomorphic coordinates $\left(X^{I}\right)$ and the tensor $h$. Alternatively, one can

\footnotetext{
${ }^{8}$ In the case $\varepsilon=1$ one may define instead a projective special para-Kähler manifold as the quotient $\hat{M}$ of $M$ by the action of the connected group $C_{0}^{*}$ which is related to $\bar{M}$ by the four-fold covering $\hat{M} \rightarrow \bar{M}$, see [3].
} 
introduce, for $X^{0} \neq 0$, inhomogeneous special $\varepsilon$-holomorphic coordinates $z^{A}=\frac{X^{A}}{X^{0}}$, where $A=1, \ldots, n$, and define an associated prepotential $\mathcal{F}\left(z^{1}, \ldots, z^{n}\right)$ by

$$
F\left(X^{0}, \ldots, X^{n}\right)=\left(X^{0}\right)^{2} \mathcal{F}\left(z^{1}, \ldots, z^{n}\right) .
$$

Then the $\varepsilon$-Kähler metric $\bar{g}$ of $\bar{M}$ has the $\varepsilon$-Kähler potential

$$
K_{\bar{g}}=-\log |Y|, \quad Y=i_{\varepsilon}\left(2(\mathcal{F}-\overline{\mathcal{F}})-\left(z^{A}-\bar{z}^{A}\right)\left(\mathcal{F}_{A}+\overline{\mathcal{F}}_{A}\right)\right),
$$

where $\mathcal{F}_{A}=\frac{\partial \mathcal{F}}{\partial z^{A}}$. We note that we can identify $\bar{M}$ locally with the submanifold $\left\{X^{0}=1\right\}$ of $M$, in particular $K_{\bar{g}}$ agrees with the pull-back of $K_{h}$ to $\left\{X^{0}=1\right\}$ up to an $\varepsilon$-Kähler transformation.

The simplest class of examples is provided by models with a quadratic prepotential

$$
F=\frac{\varepsilon i_{\varepsilon}}{4} \eta_{I J} X^{I} X^{J}
$$

where we take $\eta_{I J}$ to be real and non-degenerate. The potential for the tensor $h$ is

$$
K_{h}=-\log \left|X^{I} \eta_{I J} \bar{X}^{J}\right|
$$

Evaluating this on the hypersurface $X^{0}=1$, taking $\eta_{00}=1$, and setting $\bar{\eta}_{A B}=-\eta_{A B}$, we obtain the following $\varepsilon$-Kähler potential on $\bar{M}$ :

$$
K_{\bar{g}}=-\log \left|1-\bar{\eta}_{A B} z^{A} \bar{z}^{B}\right| .
$$

This agrees, up to an overall sign, with the $\varepsilon$-Kähler potentials for the metrics on the spaces $P(\mathcal{D})$ of $\varepsilon$-complex lines given in (2.4).

\section{$2.3 \varepsilon$-quaternionic Kähler manifolds}

\subsection{1 $\varepsilon$-quaternionic structure on a vector space}

Recall that an $\varepsilon$-quaternionic structure on a real vector space $V$ (of dimension $4 n$ ) is a Lie subalgebra $Q \subset \operatorname{End}(V)$ spanned by three pairwise anti-commuting endomorphisms $J_{1}, J_{2}, J_{3}$ that satisfy the $\varepsilon$-quaternion algebra

$$
J_{1}^{2}=J_{2}^{2}=-\varepsilon J_{3}^{2}=\varepsilon, \quad J_{1} J_{2}=J_{3} .
$$

Such a triple $\left(J_{1}, J_{2}, J_{3}\right)$ is called a standard basis of $Q$. We call $Q$ a quaternionic structure if $\varepsilon=-1$ and a para-quaternionic structure if $\varepsilon=1$. If $V$ is endowed with a pseudo-Euclidean scalar product $\langle\cdot, \cdot\rangle$ then $Q$ is called skew-symmetric if $Q$ consists of skew-symmetric endomorphisms. This implies that the signature of $\langle\cdot, \cdot\rangle$ is of the form $(4 k, 4 \ell)$, where $n=k+\ell$, if $\varepsilon=-1$ and $(2 n, 2 n)$ if $\varepsilon=1$. An orthonormal frame of $(V,\langle\cdot, \cdot\rangle, Q)$ is called an $\varepsilon$ quaternionic frame if it is of the form

$$
\left(e_{1}, \ldots, e_{n}, J_{1} e_{1}, \ldots, J_{1} e_{n}, J_{2} e_{1}, \ldots, J_{2} e_{n}, J_{3} e_{1}, \ldots, J_{3} e_{n}\right),
$$

where $\left(J_{1}, J_{2}, J_{3}\right)$ is any standard basis of $Q$ and

$$
\left\langle e_{1}, e_{1}\right\rangle=\cdots=\left\langle e_{k}, e_{k}\right\rangle=1 \text { and }\left\langle e_{k+1}, e_{k+1}\right\rangle=\cdots=\left\langle e_{k+\ell}, e_{k+\ell}\right\rangle=-1 .
$$


The Gram matrix of the basis (2.17) defines a canonical scalar product $\langle\cdot, \cdot\rangle_{\text {can }}$ on $\mathbb{R}^{4 n}$ of signature $(4 k, 4 \ell)$ if $\varepsilon=-1$ or $(2 n, 2 n)$ if $\varepsilon=1$. We will denote by $O_{\varepsilon}(4 k, 4 \ell)$ the pseudo-orthogonal group with respect to $\langle\cdot, \cdot\rangle_{\text {can }}$, and by $\mathfrak{s o}_{\varepsilon}(4 k, 4 \ell)$ its Lie algebra.

Let us denote by $J_{\alpha}^{\text {can }} \in \mathfrak{s o}_{\varepsilon}(4 k, 4 \ell)$ the matrix which represents the endomorphism $J_{\alpha}$ with respect to the basis $(2.17)$. Then

$$
Q^{\mathrm{can}}:=\operatorname{span}\left\{J_{\alpha}^{\mathrm{can}} \mid \alpha=1,2,3\right\}
$$

is a skew-symmetric $\varepsilon$-quaternionic structure on $\left(\mathbb{R}^{4 n},\langle\cdot, \cdot\rangle_{\text {can }}\right)$. The triple $\left(\mathbb{R}^{4 n},\langle\cdot, \cdot\rangle_{\text {can }}\right.$, $\left.Q^{\text {can }}\right)$ is our standard model for a pseudo-Euclidean vector space endowed with a skewsymmetric $\varepsilon$-quaternionic structure. We denote by $S p_{\varepsilon}(1)$ the group generated by the Lie algebra $\mathfrak{s p}_{\varepsilon}(1):=Q^{\text {can }}$ and by $S p_{\varepsilon}(k, \ell)$ the centraliser of $S p_{\varepsilon}(1)$ in $O_{\varepsilon}(4 k, 4 \ell)$. The Lie algebra of that centraliser is a real form of the complex Lie algebra of type $C_{n}$. The inner product and $\varepsilon$-quaternionic structure are preserved by the group

$$
S p_{\varepsilon}(1) \cdot S p_{\varepsilon}(k, \ell)= \begin{cases}\operatorname{Sp}(1) \cdot \operatorname{Sp}(k, \ell) \subset \mathrm{SO}(4 k, 4 \ell) & \text { if } \varepsilon=-1 \\ \operatorname{Sp}(2, \mathbb{R}) \cdot \operatorname{Sp}(2 n, \mathbb{R}) \subset \mathrm{SO}(2 n, 2 n) & \text { if } \varepsilon=+1\end{cases}
$$

Notice that our notation is such that $\operatorname{Sp}(k, \ell), k+\ell=n$, and $\operatorname{Sp}(2 n, \mathbb{R})$ are real forms of the same complex Lie group $\operatorname{Sp}(2 n, \mathbb{C})$.

\subsubsection{The $H \otimes E$ formalism}

Let $E=\mathbb{C}^{2 n}$ with standard basis $B_{E}=\left(\mathcal{E}_{1}, \ldots, \mathcal{E}_{2 n}\right)$. On $E$ one may define an anti-linear complex structure $j_{E}$ and non-degenerate skew-symmetric bilinear form $\omega_{E}$ that satisfy the following formulae

$$
\begin{aligned}
j_{E} \mathcal{E}_{A} & =\mathcal{E}_{A+n}, \quad j_{E} \mathcal{E}_{A+n}=-\mathcal{E}_{A}, \quad A=1, \ldots, n \\
\omega_{E} & =\sum_{A, B=1}^{n} \eta_{A B} \mathcal{E}^{A} \wedge \mathcal{E}^{B+n},
\end{aligned}
$$

where $B_{E}^{*}=\left(\mathcal{E}^{1}, \ldots, \mathcal{E}^{2 n}\right)$ is the basis of $\mathbb{C}^{2 n *}$ dual to $B_{E}$ and $\left(\eta_{A B}\right)=\operatorname{diag}\left(\mathbb{1}_{k},-\mathbb{1}_{\ell}\right)$, with $n=k+\ell$. Complex conjugation is denoted by $\rho_{E}$. The group $\operatorname{Sp}(k, \ell)$ preserves both $j_{E}$ and $\omega_{E}$, the group $\operatorname{Sp}(2 n, \mathbb{R})$ preserves both $\rho_{E}$ and $\omega_{E}$, and the symplectic form satisfies the following reality condition: $j_{E}^{*} \omega_{E}=\bar{\omega}_{E}\left(=\rho_{E}^{*} \omega_{E}\right)$.

Let $H=\mathbb{C}^{2}$ denote a specific case of the above construction, where the standard basis is denoted by $B_{H}=\left(h_{1}, h_{2}\right)$, the anti-linear complex structure by $j_{H}$, complex conjugation by $\rho_{H}$ and the bilinear form by $\omega_{H}$.

Consider the $4 n$-dimensional complex vector space $H \otimes E$ with standard basis $\left(h_{\mathcal{A}} \otimes\right.$ $\left.\mathcal{E}_{\mu}\right)_{\mathcal{A}=1,2 ; \mu=1, \ldots, 2 n}$. On $H \otimes E$ we may define the following:

(i) Two real structures $j_{H} \otimes j_{E}$ and $\rho_{H} \otimes \rho_{E}$.

(ii) A $\mathbb{C}$-bilinear scalar product $\langle\cdot, \cdot\rangle^{\mathbb{C}}=\omega_{H} \otimes \omega_{E}$. 
(iii) Three skew-symmetric endomorphisms $J_{1}, J_{2}, J_{3}$ that satisfy the $\varepsilon$-quaternion algebra and act according to

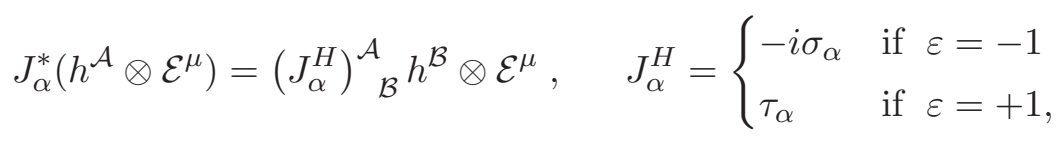

where $\sigma_{\alpha}$ are the Pauli matrices

$$
\sigma_{1}=\left(\begin{array}{ll}
0 & 1 \\
1 & 0
\end{array}\right), \quad \sigma_{2}=\left(\begin{array}{cc}
0 & -i \\
i & 0
\end{array}\right), \quad \sigma_{3}=\left(\begin{array}{cc}
1 & 0 \\
0 & -1
\end{array}\right),
$$

and

$$
\tau_{1}=\left(\begin{array}{cc}
1 & 0 \\
0 & -1
\end{array}\right), \quad \tau_{2}=\left(\begin{array}{ll}
0 & 1 \\
1 & 0
\end{array}\right), \quad \tau_{3}=\left(\begin{array}{cc}
0 & 1 \\
-1 & 0
\end{array}\right) .
$$

One may use the above data to construct an example of an $\varepsilon$-quaternionic Hermitian vector space $(V,\langle\cdot, \cdot\rangle, Q)$ of real dimension $4 n$ given by

$$
\begin{aligned}
V=(H \otimes E)^{\rho}, & \rho= \begin{cases}j_{H} \otimes j_{E} & \text { if } \varepsilon=-1 \\
\rho_{H} \otimes \rho_{E} & \text { if } \varepsilon=+1,\end{cases} \\
\langle\cdot, \cdot\rangle=\left.\langle\cdot, \cdot\rangle\right|_{V}, & Q=\left.\operatorname{span}\left\{J_{1}, J_{2}, J_{3}\right\}\right|_{V} .
\end{aligned}
$$

Since all $\varepsilon$-quaternionic Hermitian vector spaces of a given dimension are isomorphic we may state the following proposition:

Proposition 1. Let $(V,\langle\cdot, \cdot\rangle, Q)$ be an $\varepsilon$-quaternionic Hermitian vector space of real dimension $4 n$. Then we can identify $V^{\mathbb{C}}=H \otimes E$ such that the properties (2.20) are satisfied.

Indeed, a standard co-frame of $H^{*} \otimes E^{*}$ may be matched with an $\varepsilon$-quaternionic coframe of $V^{*}$ through the expressions

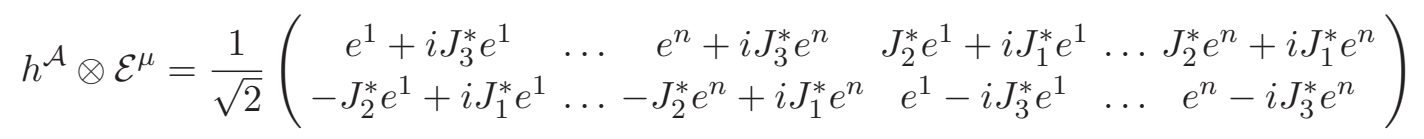

if $\varepsilon=-1$ and

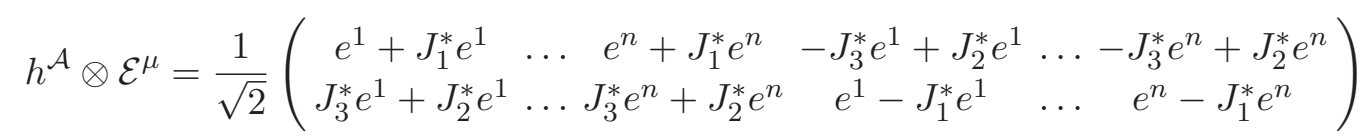

if $\varepsilon=+1$.

\subsection{3 $\varepsilon$-quaternionic structure on the tangent bundle}

The above notions can be easily transferred to vector bundles. For instance, a (fibrewise) $\varepsilon$-quaternionic structure in a vector bundle $E \rightarrow M$ is a subbundle $Q \subset \operatorname{End}(E)$ such that $Q_{p} \subset \operatorname{End}\left(E_{p}\right)$ is an $\varepsilon$-quaternionic structure on $E_{p}$ for all $p \in M$. One may 
introduce pairwise anti-commuting local sections $J_{1}, J_{2}, J_{3}$ of $Q$ defined over an open subset $U \subset M$ satisfying the $\varepsilon$-quaternion algebra, such that $Q_{p}=\operatorname{span}\left\{\left(J_{\alpha}\right)_{p} \mid \alpha=1,2,3\right\}$ for all $p \in U$. A fibre-wise $\varepsilon$-quaternionic structure on the vector bundle $T M$ is called an almost $\varepsilon$-quaternionic structure on $M$. An almost $\varepsilon$-quaternionic structure $Q$ on $M$ is called an $\varepsilon$-quaternionic structure if it is parallel with respect to a torsion-free connection, which can be characterised by the property that the covariant derivative of any section of $Q$ in the direction of any vector field is again a section of $Q$. If $M$ is endowed with a pseudoRiemannian metric then an almost $\varepsilon$-quaternionic structure on $M$ is called Hermitian if it consists of skew-symmetric endomorphisms. A pseudo-Riemannian manifold of real dimension $4 n>4$ with almost $\varepsilon$-quaternionic Hermitian structure $Q$ is called $\varepsilon$-quaternionic Kähler if $Q$ is parallel with respect to the Levi-Civita connection.

On a pseudo-Riemannian manifold $(M, g)$ with almost $\varepsilon$-quaternionic Hermitian structure we may use Proposition 1 to make the local identification $T M^{\mathbb{C}}=H \otimes E$, where $H$ and $E$ are (at least locally defined) complex vector bundles of dimension 2 and $2 n$ respectively, such that the metric and $\varepsilon$-quaternionic structure satisfy (2.20). We call a local complex co-frame of the form $\left(\mathcal{U}^{\mathcal{A} \mu}\right)=\left(h^{\mathcal{A}} \otimes \mathcal{E}^{\mu}\right)$ an $\varepsilon$-quaternionic vielbein. The metric takes the form $g=\epsilon_{\mathcal{A B}} \rho_{\mu \nu} \mathcal{U}^{\mathcal{A} \mu} \otimes \mathcal{U}^{\mathcal{B} \nu}$ and on $T^{*} M$ an $\varepsilon$-quaternionic vielbein is subject to the reality condition

$$
\overline{\mathcal{U}}^{\mathcal{A} \mu}= \begin{cases}\epsilon_{\mathcal{A} \mathcal{B}} \epsilon_{\mu \nu} \mathcal{U}^{\mathcal{B} \nu} & \text { if } \varepsilon=-1 \\ \mathcal{U}^{\mathcal{A} \mu} & \text { if } \varepsilon=+1\end{cases}
$$

where

$$
\left(\epsilon_{\mathcal{A B}}\right)=\left(\begin{array}{cc}
0 & 1 \\
-1 & 0
\end{array}\right), \quad\left(\epsilon_{\mu \nu}\right)=\left(\begin{array}{cc}
0 & \mathbb{1}_{n} \\
-\mathbb{1}_{n} & 0
\end{array}\right), \quad\left(\rho_{\mu \nu}\right)=\left(\begin{array}{cc}
0 & \eta \\
-\eta & 0
\end{array}\right) .
$$

Recall that $\eta=\left(\eta_{A B}\right)=\operatorname{diag}\left(\mathbb{1}_{k},-\mathbb{1}_{\ell}\right), k+\ell=n$. An $\varepsilon$-quaternionic vielbein may be identified with an $\varepsilon$-quaternionic co-frame through the expressions given below Proposition 1.

On a manifold with almost $\varepsilon$-quaternion structure we call an adapted connection a connection on $T M$ for which the almost $\varepsilon$-quaternionic structure is parallel. It is wellknown from the theory of $G$-structures that, with respect to a frame of the $G$-structure, the connection one-form of an adapted connection takes values in the Lie algebra $\mathfrak{g}$. An almost $\varepsilon$-quaternionic Hermitian structure corresponds to a $G$-structure with Lie group $G=S p_{\varepsilon}(1) \cdot S p_{\varepsilon}(k, \ell)$ and therefore the connection one-form of an adapted connection takes values in $\mathfrak{s p}_{\varepsilon}(1) \oplus \mathfrak{s p}_{\varepsilon}(k, \ell)$. Since this is a subalgebra of $\mathfrak{s o}_{\varepsilon}(4 k, 4 \ell)$ an adapted connection is automatically metric compatible. In an $\varepsilon$-quaternionic vielbein basis the connection one-form $\Omega$ of an adapted connection takes the form

$$
\Omega=p \otimes \mathbb{1}_{2 n}+\mathbb{1}_{2} \otimes\left(\begin{array}{cc}
q & t \\
-s & -\eta q^{T} \eta
\end{array}\right),
$$

where $p$ is a $2 \times 2$ matrix satisfying

$$
p \in \begin{cases}\mathfrak{s p}(1) \Leftrightarrow \operatorname{Tr}(p)=0, p^{\dagger}+p=0 & \text { if } \varepsilon=-1 \\ \mathfrak{s p}(2, \mathbb{R}) \Leftrightarrow \operatorname{Tr}(p)=0, p=\bar{p} & \text { if } \varepsilon=+1,\end{cases}
$$


and $q, s, t$ are $n \times n$ matrices satisfying

$$
\left(\begin{array}{cc}
q & t \\
-s-\eta q^{T} \eta
\end{array}\right) \in \begin{cases}\mathfrak{s p}(k, \ell) \Leftrightarrow t=\eta t^{T} \eta, s=\bar{t}, q^{\dagger} \eta+\eta q=0 & \text { if } \varepsilon=-1 \\
\mathfrak{s p}(2 n, \mathbb{R}) \Leftrightarrow t=\eta t^{T} \eta=\bar{t}, s=\eta s^{T} \eta=\bar{s}, q=\bar{q} & \text { if } \varepsilon=+1 .\end{cases}
$$

The coefficients of the torsion tensor are given by

$$
T^{\mathcal{A} \mu}=d \mathcal{U}^{\mathcal{A} \mu}+\Omega^{\mathcal{A} \mu}{ }_{\mathcal{B} \nu} \wedge \mathcal{U}^{\mathcal{B} \nu} .
$$

Notice that the connection matrix $\left(\Omega^{\mathcal{A} \mu}{ }_{\mathcal{B} \nu}\right)$ has the following structure, see (2.21):

$$
\Omega_{\mathcal{B} \nu}^{\mathcal{A} \mu}=\Omega_{B}^{A} \delta_{\nu}^{\mu}+\delta_{B}^{A} \Omega_{\nu}^{\mu}
$$

where $\left(\Omega_{B}^{A}\right) \in \mathfrak{s p}_{\varepsilon}(1)$ and $\left(\Omega_{\nu}^{\mu}\right) \in \mathfrak{s p}_{\varepsilon}(k, \ell)$. If the torsion vanishes then the adapted connection coincides with the Levi-Civita connection and the manifold is $\varepsilon$-quaternionic Kähler. Alternatively, if the Levi-Civita connection one-form takes values in $\mathfrak{s p}_{\varepsilon}(1) \oplus \mathfrak{s p}_{\varepsilon}(k, \ell)$ when written in an $\varepsilon$-quaternionic vielbein basis then the manifold is $\varepsilon$-quaternionic Kähler.

\section{Dimensional reduction of four-dimensional vector multiplets}

\subsection{Four-dimensional vector multiplets}

Our starting point is the bosonic part of the Lagrangian for $n=n_{V}^{(4)} \mathcal{N}=2$ vector multiplets coupled to supergravity, as given by (7.9) of [3]:

$$
\mathbf{e}_{4}^{-1} \mathcal{L}_{4}^{\left(\epsilon_{1}\right)}=\frac{1}{2} R_{4}-\bar{g}_{A B} \partial_{\hat{\mu}} z^{A} \partial^{\hat{\mu}} \bar{z}^{B}+\frac{1}{4} \mathcal{I}_{I J} F_{\hat{\mu} \hat{\nu}}^{I} F^{J \mid \hat{\mu} \hat{\nu}}+\frac{1}{4} \mathcal{R}_{I J} F_{\hat{\mu} \hat{\nu}}^{I} \tilde{F}^{J \mid \hat{\mu} \hat{\nu}} .
$$

In the following we will explain each term appearing in this expression. $R_{4}$ and $\mathbf{e}_{4}$ are the four-dimensional Ricci scalar and vielbein, and $\hat{\mu}, \hat{\nu}, \ldots$ are four-dimensional spacetime indices. We employ a notation which applies to standard (Lorentzian) and Euclidean supergravity simultaneously. The main difference between Euclidean and standard vector multiplets is that the complex structure of the scalar manifold $\bar{M}$ is replaced by a paracomplex structure $[1,3]$, and thus we use the $\varepsilon$-complex notation introduced previously. From now on the parameter $\epsilon_{1}$ distinguishes between Lorentzian space-time $\left(\epsilon_{1}=-1\right)$ and Euclidean space-time $\left(\epsilon_{1}=1\right)$.

The $\epsilon_{1}$-complex scalar fields $z^{A}$ are local coordinates of a $\mathrm{PS} \epsilon_{1} \mathrm{~K}$ manifold $M$ with metric $\bar{g}=\bar{g}_{A B} d z^{A} d \bar{z}^{B}$, where $\bar{g}_{A B}$ is the $\epsilon_{1}$-Kähler metric with $\epsilon_{1}$-Kähler potential $K_{\bar{g}}$ given in (2.16). For $\epsilon_{1}=-1$ this is the well known projective special Kähler geometry of vector multiplets in the 'new conventions' of [67], while for $\epsilon_{1}=1$ this is the projective special para-Kähler geometry of Euclidean vector multiplets which was defined in [3]. The scalar metric $\bar{g}$ has positive signature $(2 n, 0)$ for $\epsilon_{1}=-1$ and split-signature $(n, n)$ for $\epsilon_{1}=1$.

The original construction of the vector multiplet Lagrangian in Lorentzian signature was performed using the superconformal calculus [6]. This employs an auxiliary theory of $n+1$ rigid superconformal vector multiplets with complex scalars $X^{I}, I=0, \ldots, n$, which are local coordinates of a CASK manifold $M$. After gauging the superconformal transformations the theory becomes gauge equivalent to a theory of $n$ vector multiplets 
coupled to Poincaré supergravity. This construction is reviewed in [21]. The scalar metric $\bar{g}$ is obtained from the scalar metric $g=N_{I J} d X^{I} d \bar{X}^{J}$ of the scalar manifold $M$ of the associated superconformal theory by gauge fixing the local symmetry group $\mathbb{C}^{*} \simeq \mathbb{R}^{>0} \times$ $\mathrm{U}(1)$, where $\mathbb{R}^{>0}$ are dilatations, while the chiral $\mathrm{U}(1)$ transformations are part of the R-symmetry group $\mathrm{U}(1) \times \mathrm{SU}(2)$ of the $\mathcal{N}=2$ supersymmetry algebra. In [3] it was shown how this procedure can be adapted to Euclidean vector multiplets, where the scalar manifold $M$ is a conical affine special para-Kähler manifold, and where the symmetry group is $\mathbb{R}^{>0} \times \mathrm{SO}(1,1) \subset C^{*}=\mathbb{R}^{>0} \times O(1,1)$ [1]. While in this paper we find it convenient to define projective special para-Kähler manifolds by dividing out the full group $C^{*}$, only the subgroup $\mathrm{SO}(1,1) \subset O(1,1)$ is part of the R-symmetry group $\mathrm{SO}(1,1) \times \mathrm{SU}(2)$ of the Euclidean supersymmetry algebra. Consequently only the group $\mathbb{R}^{>0} \times \mathrm{SO}(1,1)$ is a symmetry of the superconformal Lagrangian. But as explained previously, dividing out the group $\mathbb{R}^{>0} \times \mathrm{SO}(1,1)$ leads to the same scalar manifold $\bar{M}$, provided that we restrict to the subset on which the function $-i_{\epsilon_{1}}\left(X^{I} \bar{F}_{I}-F_{I} \bar{X}^{I}\right)=-N_{I J} X^{I} \bar{X}^{J}$ is positive. We remark that $\mathrm{SO}(1,1) \simeq G L(1, \mathbb{R})$ is the para-unitary group $U_{\epsilon_{1}}(1), \epsilon_{1}=1$.

The relations between the superconformal theories and the supergravity theories are given by $\epsilon_{1}$-complex versions of the standard formulae of special Kähler geometry, which were presented in section 2.2. It is possible to rewrite the scalar term using $\epsilon_{1}$-complex scalar fields which are local coordinates of the associated $\mathrm{CAS} \epsilon_{1} \mathrm{~K}$ manifold $M$ :

$$
\bar{g}_{A B} \partial_{\hat{\mu}} z^{A} \partial^{\hat{\mu}} \bar{z}^{B}=\left.g_{I J} \partial_{\hat{\mu}} X^{I} \partial^{\hat{\mu}} \bar{X}^{J}\right|_{D},
$$

where the D-gauge

$$
-i_{\epsilon_{1}}\left(X^{I} \bar{F}_{I}-F_{I} \bar{X}^{I}\right)=1
$$

has been imposed. Here $g_{I J}$ are the coefficients of the pullback $h=\pi^{*} \bar{g}$ of the $\mathrm{PS} \epsilon_{1} \mathrm{~K}$ metric $\bar{g}$ to $M$, which are given by (2.13). The D-gauge restricts the scalars $X^{I}$ to a real hypersurface $S \subset M$, and since the right hand side is in addition invariant under local $U_{\epsilon_{1}}(1)$ transformations, the $n+1 \epsilon_{1}$-complex fields $X^{I}$ represent as many physical degrees of freedom as the fields $z^{A}$. While it is possible to gauge-fix the residual local $\mathrm{U}(1)_{\epsilon_{1}}$ symmetry too, we prefer not to do so at this point, because this allows us to keep all expressions manifestly covariant under symplectic transformations. The field equations of $\mathcal{N}=2$ supergravity are invariant under electric-magnetic duality transformations, which act by $\operatorname{Sp}(2 n+2, \mathbb{R})$ transformations. ${ }^{9}$ Under these transformations $\left(X^{I}, F_{I}\right)^{T}$ transforms as a vector, while the transformation of $z^{A}=X^{A} / X^{0}$ is non-linear.

The remaining two terms in (3.1) involve the abelian field strengths $F_{\hat{\mu} \hat{\nu}}^{I}$ and their Hodge-duals $\tilde{F}_{\hat{\mu} \hat{\nu}}^{I}$. As with the scalar term, the couplings $\mathcal{I}_{I J}$ and $\mathcal{R}_{I J}$ can be expressed in terms of the prepotential $F\left(X^{0}, \ldots, X^{n}\right)$. The relevant formula is

$$
\mathcal{N}_{I J}=\mathcal{R}_{I J}+i_{\epsilon_{1}} \mathcal{I}_{I J}=\bar{F}_{I J}(\bar{z})-\epsilon_{1} i_{\epsilon_{1}} \frac{(N z)_{I}(N z)_{J}}{z N z}
$$

where we defined $z^{0}=1$, and where $N_{I J}$ are the coefficients of the metric $g$ on the CAS $\epsilon_{1} \mathrm{~K}$ manifold $M$, which are given by (2.7). We use a short-hand notation where $(N z)_{I}:=N_{I J} z^{J}$ and $z N z:=z^{I} N_{I J} z^{J}$.

\footnotetext{
${ }^{9}$ We refer to [68] for a review of symplectic transformations for $\mathcal{N}=2$ vector multiplets.
} 
The negative imaginary part $-\mathcal{I}_{I J}$ of the vector coupling matrix $\mathcal{N}_{I J}$ determines the kinetic terms for the vector fields. Therefore it must be positive definite in Lorentzian space-time signature. It is known that by choosing $g=N_{I J} d X^{I} d \bar{X}^{J}$ to have signature $(2 n, 2)$, the scalar couplings $\bar{g}_{A B}$ and vector couplings $-\mathcal{I}_{I J}$ are positive definite. We remark that both $-\mathcal{I}_{I J} d X^{I} d \bar{X}^{J}$ and $g=N_{I J} d X^{I} d \bar{X}^{J}$ can be viewed as metrics on the scalar manifold $M$, and are related to one another by a simple geometric operation which flips the signature along a complex one-dimensional subspace [45].

In the Euclidean case the metric $-\mathcal{I}_{I J} d X^{I} d \bar{X}^{J}$ always has split-signature, irrespective of the signature of the real matrix $-\mathcal{I}_{I J}$. If the Euclidean theory has been obtained by dimensional reduction of a five-dimensional theory with respect to time, then the matrix $-\mathcal{I}_{I J}$ has Lorentz signature $(n, 1)$, with the negative definite direction corresponding to the Kaluza-Klein vector. The metrics $g$ and $\bar{g}$ are para-Kähler and have split-signature $(n+1, n+1)$ and $(n, n)$, respectively.

Electric magnetic duality acts on the gauge fields through the linear action of $\operatorname{Sp}(2 n+$ $2, \mathbb{R})$ on the vector $\left(F_{\hat{\mu} \hat{\nu}}^{I}, \mathcal{N}_{I J} F_{\hat{\mu} \hat{\nu}}^{J}\right)^{T}$.

\subsection{Reduction to three dimensions}

We now carry out the reduction of the four-dimensional vector multiplet Lagrangian (3.1) to three dimensions. This type of calculation is standard, so we will not give many details, though we need to specify our notation and conventions. If we start with Lorentzian signature $\left(\epsilon_{1}=-1\right)$ in four-dimensions we have the option to either reduce over space, or over time, which will be distinguished by a new parameter $\epsilon_{2}$, where $\epsilon_{2}=-1$ for spacelike reduction and $\epsilon_{2}=1$ for timelike reduction. If we start with a Euclidean theory $\left(\epsilon_{1}=1\right)$, then we can only reduce over space, so $\epsilon_{2}=-1$. All three cases will be treated simultaneously up to a certain point.

The reduction is performed along the lines of [48], with the following modifications: (i) we now include the reduction of four-dimensional Euclidean theories, (ii) some fields have been renamed, (iii) the definition of the Riemann tensor has been changed by an overall sign. For completeness, we briefly review the relation between the four-dimensional and three-dimensional quantities. Four-dimensional indices $\hat{\mu}, \hat{\nu}, \ldots$ are split into threedimensional indices $\mu, \nu, \ldots$ and the index $y$, which refers to the dimension we reduce over. We decompose the four-dimensional metric

$$
d s_{4}^{2}=-\epsilon_{2} e^{\phi}\left(d y+V_{\mu} d x^{\mu}\right)^{2}+e^{-\phi} g_{\mu \nu} d x^{\mu} d x^{\nu}
$$

into a three-dimensional metric $g_{\mu \nu}$, the Kaluza-Klein vector $V_{\mu}$ and the Kaluza-Klein scalar $\phi$. The four-dimensional vector fields have been decomposed into a scalar part $\zeta^{I}=A_{y}^{I}$ and a vector part $A_{\mu}^{I}-\zeta^{I} V_{\mu}$, with the second term restoring manifest gauge invariance. The three-dimensional field strengths $V_{\mu \nu}$ and $F_{\mu \nu}^{I}$ have then been dualised into scalars $\tilde{\phi}$ and $\tilde{\zeta}_{I}$, see [48] for details. Instead of the four-dimensional scalars $z^{A}$ we are using the corresponding superconformal scalars $X^{I}$ and the degenerate tensor $g_{I J}$. 
The resulting three-dimensional Lagrangian is

$$
\begin{aligned}
& \mathbf{e}_{3}^{-1} \mathcal{L}_{3}^{\left(\epsilon_{1}, \epsilon_{2}\right)}= \\
& \quad \frac{1}{2} R_{3}-\left.g_{I J} \partial_{\mu} X^{I} \partial^{\mu} \bar{X}^{J}\right|_{D}-\frac{1}{4} \partial_{\mu} \phi \partial^{\mu} \phi \\
& \quad+\epsilon_{1} e^{-2 \phi}\left[\partial_{\mu} \tilde{\phi}+\frac{1}{2}\left(\zeta^{I} \partial_{\mu} \tilde{\zeta}_{I}-\tilde{\zeta}_{I} \partial_{\mu} \zeta^{I}\right)\right]\left[\partial^{\mu} \tilde{\phi}+\frac{1}{2}\left(\zeta^{I} \partial^{\mu} \tilde{\zeta}_{I}-\tilde{\zeta}_{I} \partial^{\mu} \zeta^{I}\right)\right] \\
& \quad-\frac{\epsilon_{2}}{2} e^{-\phi}\left[\mathcal{I}_{I J} \partial_{\mu} \zeta^{I} \partial^{\mu} \zeta^{J}-\epsilon_{1} \mathcal{I}^{I J}\left(\partial_{\mu} \tilde{\zeta}_{I}-\mathcal{R}_{I K} \partial_{\mu} \zeta^{K}\right)\left(\partial^{\mu} \tilde{\zeta}_{J}-\mathcal{R}_{J L} \partial^{\mu} \zeta^{L}\right)\right] .
\end{aligned}
$$

For $\epsilon_{1}=\epsilon_{2}=-1$ this agrees, up to conventional choices, with [25], and for $\epsilon_{1}=-\epsilon_{2}=-1$ this agrees, up to the above mentioned changes in conventions, with [48].

As explained in [48], one can absorb the Kaluza-Klein scalar $\phi$ into the scalar fields $X^{I}$ by the field redefinition $Y^{I}=e^{\phi / 2} X^{I}$. These fields are now related to the Kaluza-Klein scalar $\phi$ by the D-gauge condition

$$
e^{\phi}=-i_{\epsilon_{1}}\left(Y^{I} \bar{F}_{I}-F_{I} \bar{Y}^{I}\right) .
$$

So $\phi$ will be now considered as a function of the independent variables $Y^{I}$. Geometrically, we interpret $\phi$ as a coordinate along the orbit of the homothetic action of $\mathbb{R}^{>0}$ on $M$. Using homogeneity, we can rewrite the scalar terms as follows:

$$
\left.g_{I J}(X) \partial_{\mu} X^{I} \partial^{\mu} \bar{X}^{J}\right|_{D}+\frac{1}{4} \partial_{\mu} \phi \partial^{\mu} \phi=g_{I J}(Y) \partial_{\mu} Y^{I} \partial^{\mu} \bar{Y}^{J}+\left.\frac{1}{4} \partial_{\mu} \phi \partial^{\mu} \phi\right|_{\phi=\phi(Y)} .
$$

Note that while both expressions take the same form, on the left hand side the fields $X^{I}$ are subject to the D-gauge, while $\phi$ is an independent field. In contrast on the right hand side $\phi$ is considered to be a dependent field, which can be expressed in terms of the $Y^{I}$. Since both sides of the equation are invariant under local $U_{\epsilon_{1}}(1)$ transformations, both sets of fields represent the same $2 n+1$ independent physical real degrees of freedom.

Now we interpret the $Y^{I}$ as $\epsilon_{1}$-holomorphic special coordinates on $M$. We can therefore rewrite the theory in terms of the associated special real coordinates $q^{a}$, defined by decomposing

$$
Y^{I}=x^{I}+u^{I}(x, y), \quad F_{I}=y_{I}+v_{I}(x, y),
$$

and setting $\left(q^{a}\right)=\left(x^{I}, y_{I}\right)^{T}$. Note that in this parametrisation the Kaluza-Klein scalar is expressed in terms of $q^{a}$ by

$$
e^{\phi}=-i_{\epsilon_{1}}\left(Y^{I} \bar{F}_{I}-F_{I} \bar{Y}^{I}\right)=-2 H,
$$

where we recall that $H$ denotes the Hesse potential. We also define $\hat{q}^{a}=\frac{1}{2}\left(\zeta^{I}, \tilde{\zeta}_{I}\right)^{T}$ and remark that both $q^{a}$ and $\hat{q}^{a}$ are symplectic vectors while the dualised Kaluza-Klein vector $\tilde{\phi}$ is a symplectic scalar. As explained in detail in [48], the Lagrangian (3.3) can be written in terms of the $4 n+5$ fields $\left(q^{a}, \hat{q}^{a}, \tilde{\phi}\right)$ with all couplings expressed using the Hesse potential $H$, the tensor field

$$
\tilde{H}_{a b}=\frac{\partial^{2}}{\partial q^{a} \partial q^{b}}\left[-\frac{1}{2} \log (-2 H)\right]=-\frac{1}{2 H} H_{a b}+\frac{1}{2 H^{2}} H_{a} H_{b},
$$


and the constant matrix $\Omega_{a b}$ representing the symplectic form (2.8):

$$
\begin{aligned}
& \mathbf{e}_{3}^{-1} \mathcal{L}_{3}^{\left(\epsilon_{1}, \epsilon_{2}\right)}= \\
& \quad \frac{1}{2} R_{3}-\tilde{H}_{a b}\left(\partial_{\mu} q^{a} \partial^{\mu} q^{b}-\epsilon_{2} \partial_{\mu} \hat{q}^{a} \partial^{\mu} \hat{q}^{b}\right) \\
& \quad+\epsilon_{1} \frac{1}{H^{2}}\left(q^{a} \Omega_{a b} \partial_{\mu} q^{b}\right)\left(q^{a} \Omega_{a b} \partial^{\mu} q^{b}\right) \\
& \quad-\epsilon_{1} \epsilon_{2} \frac{2}{H^{2}}\left(q^{a} \Omega_{a b} \partial_{\mu} \hat{q}^{b}\right)\left(q^{a} \Omega_{a b} \partial^{\mu} \hat{q}^{b}\right) \\
& \quad+\epsilon_{1} \frac{1}{4 H^{2}}\left(\partial_{\mu} \tilde{\phi}+2 \hat{q}^{a} \Omega_{a b} \partial_{\mu} \hat{q}^{b}\right)\left(\partial^{\mu} \tilde{\phi}+2 \hat{q}^{a} \Omega_{a b} \partial^{\mu} \hat{q}^{b}\right) .
\end{aligned}
$$

Since all local degrees of freedom have been converted into scalars, the Lagrangian (3.5) is a non-linear sigma model coupled to gravity. The $4 n+5$ real scalar fields $\left(q^{a}, \hat{q}^{a}, \tilde{\phi}\right)$ are local coordinates of its target space $P$. Due to the local $U_{\epsilon_{1}}(1)$ symmetry, there are only $4 n+4$ physical degrees of freedom, and the symmetric tensor field on $P$ defined by the Lagrangian is invariant and degenerate along the orbits of the $U_{\epsilon_{1}}(1)$-action. By gauge-fixing this symmetry we can obtain a sigma model with a $(4 n+4)$-dimensional target manifold $\bar{N}$, equipped with a non-degenerate metric. Since $U_{\epsilon_{1}}(1)$ acts on the symplectic vector $q^{a}$, while $\hat{q}^{a}$ and $\tilde{\phi}$ are invariant, such a gauge fixing will break the manifest symplectic invariance of the sigma model with target $P$. Therefore it is advantageous to describe $\bar{N}$ in terms of the larger space $P$.

In the following sections we will show that $P$ is a principal $U_{\epsilon_{1}}(1)$-bundle over $\bar{N}$, and that the degenerate symmetric tensor on $P$ defined by the Lagrangian (3.5) projects down to an $\varepsilon$-quaternionic Kähler metric on $\bar{N}$. For $\epsilon_{1}=\epsilon_{2}=-1$ we recover the result of [25], in which it was shown that $\bar{N}$ is quaternionic Kähler. For $\epsilon_{1}=1, \epsilon_{2}=-1$ and $\epsilon_{1}=-1$, $\epsilon_{2}=1$, we prove that the manifold $\bar{N}$ is para-quaternionic Kähler.

\section{Global construction of the $c$-map}

\subsection{Geometric data on a conic affine special $\varepsilon$-Kähler manifold}

The starting point for our construction of the $c$-map will be a regular, simply connected $\mathrm{CAS} \varepsilon \mathrm{K}$ manifold $M$, see section 2.2. The purpose of this section is to introduce a global orthogonal co-frame on $M$ and to express certain geometrical data in terms of this coframe. We are specifically interested in the cubic tensor $C=\nabla g$, the difference tensor $S=D-\nabla$, and the pull-back of the Levi-Civita connection one-form on the corresponding PS $\varepsilon \mathrm{K}$ manifold $\sigma=\pi^{*} \bar{\sigma}$. The necessary expressions are given by (4.11), (4.12) and (4.14) respectively.

Let $(M, J, g, \nabla, \xi)$ be a regular, simply connected CASEK manifold of dimension $2 n+2$, which in the case $\varepsilon=-1$ has signature $(2 k, 2 \ell+2), k+\ell=n$. Recall that $M$ is a principal $\mathbb{C}_{\varepsilon}^{*}$-bundle over a PS $\varepsilon \mathrm{K}$ manifold $(\bar{M}, \bar{J}, \bar{g})$ of dimension $2 n$, with fundamental vector fields

$$
\xi=q^{a} \frac{\partial}{\partial q^{a}}, \quad J \xi=\frac{1}{2} H_{b} \Omega^{b a} \frac{\partial}{\partial q^{a}} .
$$


For the case $\varepsilon=-1$ the signature of $\bar{M}$ is $(2 k, 2 \ell)$. The tangent space at any point $p \in M$ decomposes into horizontal and vertical parts $T_{p} M=\mathcal{H}_{p} \oplus \operatorname{span}\left\{\xi_{p}, J \xi_{p}\right\}$, where the horizontal space $\mathcal{H}_{p}$ is defined as the orthogonal complement of the vertical space of $\pi: M \rightarrow \bar{M}$ and we identify $\mathcal{H}_{p} \simeq T_{\pi(p)} \bar{M}$ by the projection. Recall that there exists a global $\nabla$-affine symplectic frame on $M$ given by $\mathcal{B}=\left(\frac{\partial}{\partial q^{0}}, \ldots, \frac{\partial}{\partial q^{2 n+1}}\right)$, which is unique up to $\operatorname{Sp}(2 n+2, \mathbb{R})$ transformations. Recall also that the projective special $\varepsilon$-Kähler metric on $\bar{M}$ is induced by the degenerate tensor $h$ defined in equation (2.12). Therefore the horizontal lift of an orthonormal frame defined over an open subset $U \subset \bar{M}$ yields an $h$-orthonormal frame $\mathcal{B}^{\prime}=\left(e_{1}, \ldots, e_{2 n}\right)$ of $\mathcal{H}$, defined over $\pi^{-1}(U)$, such that

$$
h\left(e_{m}, e_{p}\right)=\eta_{m p}, \quad m, p=1, \ldots, 2 n
$$

where

$$
\left(\eta_{m p}\right)=\left(\begin{array}{cc}
d & 0 \\
0 & -\varepsilon d
\end{array}\right), \quad d= \begin{cases}\operatorname{diag}\left(\mathbb{1}_{k},-\mathbb{1}_{\ell}\right), & \varepsilon=-1 \\
\operatorname{diag}\left(\mathbb{1}_{n}\right), & \varepsilon=+1 .\end{cases}
$$

Moreover, by choosing the orthonormal frame on $U \subset \bar{M}$ adapted to the $\varepsilon$-complex structure we can further assume that

$$
J\left(e_{A}\right)=e_{A+n}, \quad J\left(e_{A+n}\right)=\varepsilon e_{A}, \quad A=1, \ldots, n .
$$

In such a frame the $\varepsilon$-complex structure $J\left(e_{p}\right)=J^{m}{ }_{p} e_{m}$ is represented by the constant matrix

$$
\left(J_{p}^{m}\right)=\left(\begin{array}{cc}
0 & \varepsilon \mathbb{1} \\
\mathbb{1} & 0
\end{array}\right) .
$$

Such a choice of frame is not unique, with any two choices differing by a gauge transformation with values in

$$
U_{\varepsilon}(k, \ell) \subset S O_{\varepsilon}(2 k, 2 \ell):= \begin{cases}\mathrm{SO}(2 k, 2 \ell), & \varepsilon=-1 \\ \mathrm{SO}(n, n), & \varepsilon=+1, \quad n=k+\ell .\end{cases}
$$

We consider the frame $\mathcal{B}^{\prime *}=\left(e^{1}, \ldots, e^{2 n}\right)$ of $\mathcal{H}^{*}=\operatorname{span}\{\xi, J \xi\}^{0} \subset T^{*} M$ dual to $\mathcal{B}^{\prime}$; that is $e^{m}\left(e_{p}\right)=\delta_{p}^{m}$. (Here the superscript 0 refers to the annihilator.) Note that the $(0,2)$-tensor $h$ takes the form $h=\eta_{m p} e^{m} \otimes e^{p}$. Similarly, on $\operatorname{span}\{\xi, J \xi\}^{*}=\mathcal{H}^{0}$ one may introduce the frame $(u, v)$ given by

$$
u=\frac{1}{2 H} d H, \quad v=\varepsilon J^{*} u=-\frac{1}{H} q^{a} \Omega_{a b} d q^{b},
$$

which is dual to $(\xi, J \xi)$.

Consider the orthogonal projection $\varphi: T M \rightarrow \mathcal{H}$. Using the frame $\mathcal{B}$ of $T M$ and the local frame $\mathcal{B}^{\prime}$ of $\mathcal{H}$ this projection is represented by the matrix

$$
M_{\mathcal{B}, \mathcal{B}^{\prime}}(\varphi)=\mathcal{P}=\left(\mathcal{P}^{m}\right)_{a=0, \ldots, 2 n+1}^{m=1, \ldots, 2 n} .
$$


One may also consider the corresponding dual map $\varphi^{*}: \mathcal{H}^{*} \rightarrow T^{*} M$, which with the above identifications is simply the inclusion map. Using the dual basis $\mathcal{B}^{*}=\left(d q^{0}, \ldots, d q^{2 n+1}\right)$ of $T^{*} M$ and $\mathcal{B}^{*}$ of $\mathcal{H}^{*}$ this map is represented by the transposed matrix

$$
M_{\mathcal{B}^{*}, \mathcal{B}^{*}}\left(\varphi^{*}\right)=\mathcal{P}^{t}=\left(\mathcal{P}_{a}{ }^{m}\right)_{a=0, \ldots, 2 n+1}^{m=1, \ldots, 2 n},
$$

in other words

$$
e^{m}=\mathcal{P}_{a}^{m} d q^{a} .
$$

It is useful to consider also the inclusion map $\iota: \mathcal{H} \rightarrow T M$, which is characterised by

$$
\varphi \circ \iota=\operatorname{Id}_{\mathcal{H}}, \quad \varphi^{\perp} \circ \iota=0,
$$

where $\varphi^{\perp}: T M \rightarrow \mathcal{H}^{\perp}=\operatorname{span}\{\xi, J \xi\}$ is the orthogonal projection onto $\mathcal{H}^{\perp}$.

Lemma 1. The matrix $M_{\mathcal{B}^{\prime}, \mathcal{B}}(\iota)$ representing the inclusion $\iota: \mathcal{H} \rightarrow T M$ is given by

$$
\mathcal{T}=\left(\mathcal{T}^{a}{ }_{m}\right)_{a=0, \ldots, 2 n+1}^{m=1, \ldots, 2 n}, \quad \mathcal{T}_{m}^{a}=-2 H H^{a b} \mathcal{P}^{p}{ }_{b} \eta_{p m}
$$

We have the equation

$$
e_{m}=\mathcal{T}_{m}^{a} \frac{\partial}{\partial q^{a}},
$$

and the matrix $\mathcal{P}^{t}$ has the following properties:

(ii) $\quad \eta_{m p}\left(\mathcal{P}_{a}{ }^{m} \mathcal{P}_{b}{ }^{p}\right)=h_{a b}=-\frac{1}{2 H} H_{a b}+\frac{1}{4 H^{2}} H_{a} H_{b}-\varepsilon \frac{1}{H^{2}} \Omega_{a c} q^{c} \Omega_{b d} q^{d}$.

(iii) $\quad-2 H \mathcal{P}_{a}{ }^{m} H^{a b} \mathcal{P}_{b}{ }^{p}=\eta^{m p}$.

(iv) $\quad H\left(\mathcal{P}_{a}{ }^{m} \Omega^{a b} \mathcal{P}_{b}{ }^{q}\right) \eta_{q p}=J_{p}^{*}{ }^{m}=J_{p}^{m}$,

where $\left(J_{p}^{m}\right)$ is the constant matrix (4.2) representing the tensor $\left.J\right|_{\mathcal{H}}: \mathcal{H} \rightarrow \mathcal{H}$ in the frame $\left(e_{m}\right)$.

Proof. Part (i) follows immediately from the fact that $\operatorname{ker} \varphi=\operatorname{span}\{\xi, J \xi\}$. For part (ii) one may use the fact that

$$
h=\varphi^{*} h=\eta_{m p}\left(\mathcal{P}_{a}{ }^{m} \mathcal{P}_{b}{ }^{p}\right) d q^{a} \otimes d q^{b},
$$

and therefore $\eta_{m p}\left(\mathcal{P}_{a}{ }^{m} \mathcal{P}_{b}{ }^{p}\right)=h_{a b}$, cf. (2.14). For part (iii) we note that $-\frac{1}{2 H} g$ and $h$ coincide when restricted to $\mathcal{H}$

$$
-\left.\frac{1}{2 H} g\right|_{\mathcal{H}}=\left.h\right|_{\mathcal{H}} .
$$

Since this is non-degenerate on $\mathcal{H}$ we can invert this formula

$$
-2 H g^{-1} \circ\left(\varphi^{*}, \varphi^{*}\right)=-\left.2 H g^{-1}\right|_{\mathcal{H}^{*}}=-2 H\left(\left.g\right|_{\mathcal{H}}\right)^{-1}=\left(\left.h\right|_{\mathcal{H}}\right)^{-1}=\eta^{m n} e_{m} \otimes e_{n} .
$$

Plugging in $\left(e^{m}, e^{p}\right)$ gives expression (iii). Using (iii) one can easily check that $\mathcal{T}$ satisfies the equation

$$
\mathcal{P}_{a}^{m} \mathcal{T}_{p}^{a}=\delta_{p}^{m}
$$


Using (i) one can also check that the vectors $\mathcal{T}_{m}^{a} \frac{\partial}{\partial q^{a}}$ are perpendicular to $\xi$ and $J \xi$. In view of the characterisation (4.5), this proves that the matrix $\mathcal{T}$ represents the inclusion map $\iota: \mathcal{H} \rightarrow T M$. The latter property implies (4.7).

For part (iv) we note that $\varphi^{*} \circ J^{*}=J^{*} \circ \varphi^{*}: \mathcal{H}^{*} \rightarrow T^{*} M$. Acting on $e^{m}$ this gives

$$
J_{p}^{*}{ }^{m} e^{p}=\mathcal{P}_{a}{ }^{m} J^{*}{ }_{b}^{a} d q^{b} .
$$

Plugging $e_{n}$ into this expression and using (4.7), (4.6) and (2.9) gives the desired result.

Let us now turn our attention to the cubic tensor $C=\nabla g=H_{a b c} d q^{a} \otimes d q^{b} \otimes d q^{c}$, where $H_{a b c}$ are the triple derivatives of the Hesse potential. The cubic tensor is related to the difference tensor

$$
S=D-\nabla=\frac{\varepsilon}{2} J \nabla J
$$

by the formula

$$
C(X, Y, Z)=2 g\left(S_{X} Y, Z\right)
$$

which is immediately obtained from $g=\omega(J \cdot, \cdot)$. Differentiating the equation $g(J X, J Y)=$ $\varepsilon g(X, Y)$ with respect to $\nabla$ and using the equation (4.9) one can prove that

$$
C(\cdot, J \cdot, J \cdot)=\varepsilon C(\cdot, \cdot, \cdot) \text {. }
$$

The cubic tensor is degenerate with kernel containing $\operatorname{span}\{\xi, J \xi\}$ but not $\mathbb{C}_{\varepsilon}^{*}$-invariant, and therefore not well-defined on $\bar{M}$. In the above local frame of $\mathcal{H}^{*} \subset T^{*} M$ we may write $C=C_{m p q} e^{m} \otimes e^{p} \otimes e^{q}$, where the components are symmetric and satisfy

$$
C_{m p q}=\sum_{a, b, c=0}^{2 n+1} \mathcal{T}_{m}^{a} \mathcal{T}_{p}^{b} \mathcal{T}_{q}^{c} H_{a b c}
$$

Due to (4.9) the components of the difference tensor $S=S_{p q}^{m} e_{m} \otimes e^{p} \otimes e^{q}$ are given by

$$
S_{p q}^{m}=-\frac{1}{4 H} \eta^{m r} C_{r p q}
$$

and the one-forms $X \mapsto S^{m}{ }_{p}(X)=e^{m}\left(S_{X} e_{p}\right)$ by

$$
S^{m}{ }_{p}=\sum_{q=1}^{2 n} S^{m}{ }_{p q} e^{q}=\sum_{q=1}^{2 n} \sum_{a, b=0}^{2 n+1}-\frac{1}{4 H} \eta^{m q} \mathcal{T}_{q}^{a} \mathcal{T}_{p}^{b} d H_{a b}
$$

It follows from (4.10) that

$$
\begin{aligned}
\left(S_{B}^{A}\right) & =d\left(S_{B}^{A}\right)^{T} d, & S_{B}^{A} & =-S_{B+n}^{A+n}, \\
\left(S_{B}^{A+n}\right) & =d\left(S_{B}^{A+n}\right)^{T} d, & S_{B}^{A+n} & =-\varepsilon S_{B+n}^{A} .
\end{aligned}
$$

We end this section by computing the pull-back to $M$ of the Levi-Civita connection one-form on the corresponding PSeK manifold $\bar{M}$. 
Lemma 2. Let $\sigma \in \Omega^{1}\left(M, \mathfrak{s} o_{\varepsilon}(k, \ell)\right)$ denote the pull-back of the Levi-Civita connection one-form $\bar{\sigma}$ on $\bar{M}$. The components of $\sigma$ in the above local frame of $\mathcal{H}^{*} \subset T^{*} M$ are given by

$$
\sigma_{p}^{m}=-v J_{p}^{m}+H\left(d \mathcal{P}_{a}{ }^{m} H^{a b} \mathcal{P}_{b}{ }^{q}-\mathcal{P}_{a}{ }^{m} H^{a b} d \mathcal{P}_{b}{ }^{q}\right) \eta_{q p}
$$

where the one-form $v$ was defined in (4.3).

Proof. The Levi-Civita connection one-form $\bar{\sigma}$ on $\bar{M}$ is uniquely determined by the requirement that it is metric compatible and torsion-free. In terms of the pull-back $\sigma$ the metric compatibility condition implies that

$$
\left(\sigma_{p}^{m}\right) \in\left\{\begin{array}{ll}
\Omega^{1}(M, \mathfrak{s o}(2 k, 2 \ell)), & \varepsilon=-1 \\
\Omega^{1}(M, \mathfrak{s o}(n, n)), & \varepsilon=+1
\end{array} \quad \Leftrightarrow \quad \sigma^{m p}=\sigma^{m}{ }_{q} \eta^{q p}=-\sigma^{p m}\right.
$$

which is easily seen to be satisfied by (4.14). The torsion-free condition implies that

$$
d e^{m}+\sigma_{p}^{m} \wedge e^{p}=0
$$

which we will now show is satisfied by (4.14). Using Lemma 1 (iii) and (iv) we have

$$
\begin{aligned}
\sigma_{p}^{m} \wedge e^{p}= & -(d q \Omega q) \wedge \mathcal{P}_{a}{ }^{m} \Omega^{a b} \mathcal{P}_{b}{ }^{p} \eta_{p q} e^{q}-\frac{1}{2 H} d H \wedge e^{m} \\
& +\underbrace{2 H d \mathcal{P}_{a}{ }^{m} H^{a b} \mathcal{P}_{b}{ }^{p} \eta_{p q} \wedge e^{q}}_{(*)}+\underbrace{H \mathcal{P}_{a}{ }^{m} d H^{a b} \mathcal{P}_{b}{ }^{p} \eta_{p q} \wedge e^{q}}_{S^{m}{ }_{p} \wedge e^{p}=0} .
\end{aligned}
$$

To calculate the last term we have used (4.12) and (4.6). It vanishes in virtue of the symmetry of $S$. Calculating (*) individually using (2.10), (4.4) and Lemma 1 (i-ii) we find

$$
\begin{aligned}
(*) & =2 H d \mathcal{P}_{a}{ }^{m} H^{a b} \wedge\left[-\frac{H_{b c}}{2 H}+\frac{H_{b} H_{c}}{4 H^{2}}-\varepsilon \frac{\Omega_{b d} q^{d} \Omega_{c e} q^{e}}{H^{2}}\right] d q^{c} \\
& =-d \mathcal{P}_{a}{ }^{m} \wedge d q^{a}+\frac{1}{2 H} d \mathcal{P}_{a}{ }^{m} q^{a} \wedge d H-\varepsilon \frac{2}{H} d \mathcal{P}_{a}{ }^{m} H^{a b} \Omega_{b c} q^{c} \wedge(d q \Omega q) \\
& =-d e^{m}+\frac{1}{2 H} d H \wedge e^{m}+(d q \Omega q) \wedge \mathcal{P}_{a}{ }^{m} \Omega^{a b} \mathcal{P}_{b}{ }^{p} \eta_{p q} e^{q}
\end{aligned}
$$

and therefore expression (4.16) is satisfied.

By Proposition 3 of section 5 the solution to (4.15) and (4.16) is unique, and, moreover, it is precisely the pull-back of the Levi-Civita connection one-form on $\bar{M}$.

For an $\varepsilon$-Kähler manifold the Levi-Civita one-form satisfies $J^{*} \circ \sigma=\sigma \circ J$. It follows that

$$
\begin{aligned}
\left(\sigma_{B}^{A}\right) & =-d\left(\sigma_{B}^{A}\right)^{T} d, & \sigma_{B}^{A} & =\sigma^{A+n}{ }_{B+n}, \\
\left(\sigma_{B}^{A+n}\right) & =d\left(\sigma_{B}^{A+n}{ }_{B}\right)^{T} d, & \sigma_{B}^{A+n}{ }_{B} & =\varepsilon \sigma_{B+n}^{A} .
\end{aligned}
$$




\subsection{The $c$-map for various spacetime signatures}

In this section we will construct the $c$-map target manifold $\left(\bar{N}, g_{\bar{N}}, Q_{\bar{N}}\right)$. We will present this construction for the spatial, temporal and Euclidean $c$-maps in a unified way using the $\left(\epsilon_{1}, \epsilon_{2}\right)$-notation introduced previously. We will begin with the topological data on $\bar{N}$, before moving on to the metric $g_{\bar{N}}$ and $\varepsilon$-quaternionic structure $Q_{\bar{N}}$.

Consider a regular, simply connected $\mathrm{CAS} \epsilon_{1} \mathrm{~K}$ manifold $M$ of dimension $2 n+2$. Given $M$ one may construct the $(4 n+5)$-dimensional manifold $P=T M \times \mathbb{R}$, that is the product of the tangent bundle of $M$ with the real line. On $P$ we have $4 n+5$ global real functions $\left(q^{a}, \hat{q}^{b}, \tilde{\phi}\right)$ which are defined as follows. We start with the globally-defined functions $\left(q^{a}\right)$ on $M$, introduced before, which restrict to special real coordinates in a neighbourhood $U$ of any point of $M$. The function $\hat{q}^{b}$ on $T M$ is defined by the property that it takes the value $v^{b}$ on the vector $v^{a} \frac{\partial}{\partial q^{a}}$. As we have natural projections $P \rightarrow T M$ and $T M \rightarrow M$, the functions $q^{a}, \hat{q}^{b}$, can be considered as functions on $P$. Finally $\tilde{\phi}$ is the coordinate on the $\mathbb{R}$-factor. Notice that the functions $\left(q^{a}, \hat{q}^{b}, \tilde{\phi}\right)$ restrict to a local coordinate system in a neighbourhood of any point of $P$, in fact, they give coordinates on the open set $p^{-1}(U) \times \mathbb{R} \subset P$.

The principal $\mathbb{C}_{\epsilon_{1}}^{*}$-action on $M$ may be lifted to a principal $\mathbb{C}_{\epsilon_{1}}^{*}$-action on $P$ in the following way. Using the global frame $\left(\frac{\partial}{\partial q^{a}}\right)$ we can identify the vector bundle $T M$ with the trivial bundle $M \times \mathbb{R}^{2 n+2}$ and extend the principal $\mathbb{C}_{\varepsilon}^{*}$-action on $M$ trivially to a principal $\mathbb{C}_{\varepsilon}^{*}$-action on $T M$ and $P=T M \times \mathbb{R}$. The actions $\varphi_{\lambda}^{M}$ and $\varphi_{\lambda}^{P}$ of an element $\lambda \in \mathbb{C}_{\varepsilon}^{*}$ on $M$ and $P$ are related by

$$
\left(\varphi_{\lambda}^{P}\right)^{*} q^{a}=\operatorname{pr}^{*}\left(\varphi_{\lambda}^{M}\right)^{*}\left(\left.q^{a}\right|_{M}\right), \quad\left(\varphi_{\lambda}^{P}\right)^{*} \hat{q}^{a}=\hat{q}^{a}, \quad\left(\varphi_{\lambda}^{P}\right)^{*} \tilde{\phi}=\tilde{\phi} .
$$

On $P$ one may consider the principal action of the subgroup $U_{\epsilon_{1}}(1) \subset \mathbb{C}_{\epsilon_{1}}^{*}$. In this way, one may interpret $P$ as a principal $U_{\epsilon_{1}}(1)$ bundle over a manifold $\bar{N}$. Let $Z_{P}$ be the vector field generating the infinitesimal $U_{\epsilon_{1}}(1)$-action on $P$. This is precisely the horizontal lift of the vector field $J \xi$ on $M$, and is given by

$$
Z_{P}=\frac{1}{2} H_{a} \Omega^{a b} \frac{\partial}{\partial q^{b}}
$$

We define the $c$-map target manifold as the orbit space

$$
\bar{N}=P / U_{\epsilon_{1}}(1)
$$

which by construction has dimension $4 n+4$. This information is summarised in figure 1 . Notice that in the case $\epsilon_{1}=1$ the manifold $\bar{N}$ has at least two connected components distinguished by the sign of the Hesse potential $H$.

The non-linear sigma model of the dimensionally reduced Lagrangian (3.5) defines on $P$ the symmetric bilinear form

$$
\begin{aligned}
g^{\prime}= & \tilde{H}_{a b}\left(d q^{a} \otimes d q^{b}-\epsilon_{2} d \hat{q}^{a} \otimes d \hat{q}^{b}\right) \\
& -\epsilon_{1} \frac{1}{H^{2}}\left(q^{a} \Omega_{a b} d q^{b}\right) \otimes\left(q^{a} \Omega_{a b} d q^{b}\right)+\epsilon_{1} \epsilon_{2} \frac{2}{H^{2}}\left(q^{a} \Omega_{a b} d \hat{q}^{b}\right) \otimes\left(q^{a} \Omega_{a b} d \hat{q}^{b}\right) \\
& -\epsilon_{1} \frac{1}{4 H^{2}}\left(d \tilde{\phi}+2 \hat{q}^{a} \Omega_{a b} d \hat{q}^{b}\right) \otimes\left(d \tilde{\phi}+2 \hat{q}^{a} \Omega_{a b} d \hat{q}^{b}\right),
\end{aligned}
$$




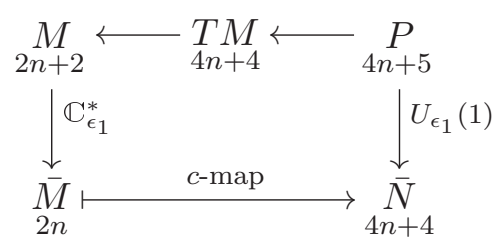

Figure 1. The global construction of the $c$-map target manifold.

where $\epsilon_{1}$ and $\epsilon_{2}$ are determined by the different $c$-maps according to the rule (1.2) and where $H \neq 0$ is now allowed to change sign and the $\mathrm{PS} \epsilon_{1} \mathrm{~K}$ metric is allowed to be indefinite. The bilinear form $g^{\prime}$ has a one-dimensional kernel $\mathbb{R} Z_{P}$ and is invariant under the $U_{\epsilon_{1}}(1)$-action on $P$. It may therefore be pushed-down to give a well-defined metric $g_{\bar{N}}$ on $\bar{N}$. This procedure makes sense even in the case $\epsilon_{1}=\epsilon_{2}=1$, which we have not given a physical interpretation so far. As the next proposition shows, this case gives a metric equivalent to the one in the case $\epsilon_{1}=-\epsilon_{2}=1$.

Proposition 2. For the case $\epsilon_{1}=1$ the pull-back of $g^{\prime}$ under the action of $e \in C^{*}$ is given by

$$
\left(\varphi_{e}^{P}\right)^{*}\left(\left.g^{\prime}\right|_{\left(\epsilon_{1}, \epsilon_{2}\right)=(1,1)}\right)=\left.g^{\prime}\right|_{\left(\epsilon_{1}, \epsilon_{2}\right)=(1,-1)} .
$$

Proof. The pull-back of the functions $\left(q^{a}, \hat{q}^{b}, \tilde{\phi}\right)$ are given by

$$
\left(\varphi_{e}^{P}\right)^{*} q^{a}=\epsilon_{1} J_{b}^{a} q^{b}, \quad\left(\varphi_{e}^{P}\right)^{*} \hat{q}^{a}=\hat{q}^{a}, \quad\left(\varphi_{e}^{P}\right)^{*} \tilde{\phi}=\tilde{\phi}
$$

cf. (4.18). In fact, the first term is computed as follows using (2.15):

$$
\left(\varphi_{e}^{P}\right)^{*}\left(\begin{array}{c}
x^{I} \\
y_{J}
\end{array}\right)=\left(\begin{array}{c}
u^{I} \\
v_{J}
\end{array}\right)=\left(-\epsilon_{1} \frac{1}{2} \Omega^{a b} H_{b}\right)=\left(-\epsilon_{1} \frac{1}{2} \Omega^{a b} H_{b c} q^{c}\right)=\left(\epsilon_{1} J_{b}^{a} q^{b}\right)
$$

where $J_{b}^{a}$ are the components of the para-complex structure on $M$, pulled-back to $P$. Notice that from this calculation we also obtain

$$
\left(\varphi_{e}^{P}\right)^{*} H_{a}=-\epsilon_{1} 2 \Omega_{a b} q^{b} .
$$

Using these formulae together with the identities $\left(\varphi_{e}^{P}\right)^{*} H=-H$ and $\left(\varphi_{e}^{P}\right)^{*} H_{a b}=H_{a b}$, which follow from the fact that $e$ acts anti-isometrically on the metric $g$ of $M$, the result is easy to check.

Recall that the manifold $\left(\bar{N}, g_{\bar{N}}\right)$ is obtained by taking the quotient of $P$ with respect to the action of $U_{\epsilon_{1}}(1) \subset \mathbb{C}_{\epsilon_{1}}^{*}$. In the case $\epsilon_{1}=1$ the action of $e \notin U_{\epsilon_{1}}(1)$ on $P$ induces an involution on $\bar{N}$ which interchanges the connected components of $\bar{N}$. This $\mathbb{Z}_{2}$-action does not preserve the metric $g_{\bar{N}}$, but maps $\left(\bar{N},\left.g_{\bar{N}}\right|_{\left(\epsilon_{1}, \epsilon_{2}\right)=(1,-1)}\right)$ to $\left(\bar{N},\left.g_{\bar{N}}\right|_{\left(\epsilon_{1}, \epsilon_{2}\right)=(1,1)}\right)$ and therefore both manifolds are globally isometric. For this reason one may take either $\epsilon_{2}=-1$ or $\epsilon_{2}=+1$ for the Euclidean $c$-map at the expense of working with a manifold $\bar{N}$ which is not connected but naturally contains both possible choices. 


\subsubsection{A co-frame of $P$ adapted to the pull back of the $c$-map metric}

On a local patch of $P$ it is convenient to introduce the following $4 n+4$ linearly independent one-forms:

$$
\begin{aligned}
e^{m} & =\mathcal{P}_{a}{ }^{m} d q^{a}, & \hat{e}^{m} & =\mathcal{P}_{a}{ }^{m} d \hat{q}^{a}, \\
u^{1} & =\frac{1}{2 H} H_{a} d q^{a}, & \hat{u}^{1} & =-\frac{1}{2 H} H_{a} d \hat{q}^{a}, \\
u^{2} & =-\frac{1}{2 H}\left(d \tilde{\phi}+2 \hat{q}^{a} \Omega_{a b} d \hat{q}^{b}\right), & \hat{u}^{2} & =-\frac{1}{H} q^{a} \Omega_{a b} d \hat{q}^{b} .
\end{aligned}
$$

We will refer to the collection $L^{*}=\left(e^{m}, u^{1}, u^{2}, \hat{e}^{n}, \hat{u}^{1}, \hat{u}^{2}\right)_{m, n=1, \ldots, 2 n}$ as a local partial coframe on $P$. Note that $Z_{P}^{0}=\operatorname{span} L^{*}$. The globally-defined one-forms $u^{1}, u^{2}, \hat{u}^{1}, \hat{u}^{2} \in L^{*}$ are independent of the choice of the functions $q^{a}$ and therefore uniquely defined. The oneforms $e^{m}, \hat{e}^{n} \in L^{*}$ are unique up to $U_{\epsilon_{1}}(k, \ell) \subset S O_{\epsilon_{1}}(2 k, 2 \ell)$ gauge transformations, which act according to

$$
e^{m} \mapsto A_{n}^{m} e^{n}, \quad \hat{e}^{m} \mapsto A_{n}^{m} \hat{e}^{n}, \quad\left(A_{n}^{m}\right) \in U_{\epsilon_{1}}(k, \ell) .
$$

The bilinear form is written in terms of the partial co-frame $L^{*}$ as

$$
g^{\prime}=\eta_{m p} e^{m} \otimes e^{p}+u^{1} \otimes u^{1}-\epsilon_{1} u^{2} \otimes u^{2}-\epsilon_{2}\left[\eta_{m p} \hat{e}^{m} \otimes \hat{e}^{p}+\hat{u}^{1} \otimes \hat{u}^{1}-\epsilon_{1} \hat{u}^{2} \otimes \hat{u}^{2}\right],
$$

where $\left(\eta_{m p}\right)$ is given by (4.1). Consider the globally-defined one-form

$$
v=-\frac{1}{H} q^{a} \Omega_{a b} d q^{b} .
$$

This satisfies $v\left(Z_{P}\right)=1$ and is invariant with respect to the $U_{\epsilon_{1}}(1)$-action on $P$. Therefore it may be interpreted as a connection on the principal $U_{\epsilon_{1}}(1)$-bundle $P \rightarrow \bar{N}$. We extend the partial local co-frame $L^{*}$ to a local co-frame $\left(L^{*}, v\right)$ on $P$.

It is important to note that although $g^{\prime}$ is invariant under the $U_{\epsilon_{1}}(1)$-action this is not necessarily true for the individual one-forms in $L^{*}$. In fact, only $e^{m}, u^{1}, u^{2}$ are invariant under the action of $U_{\epsilon_{1}}(1)$, with the remaining one-forms transforming according to

$$
\mathcal{L}_{Z_{P}} \hat{e}^{A}=-\epsilon_{1} \hat{e}^{A+n}, \quad \mathcal{L}_{Z_{P}} \hat{e}^{A+n}=-\hat{e}^{A}, \quad \mathcal{L}_{Z_{P}} \hat{u}^{1}=\epsilon_{1} \hat{u}^{2}, \quad \mathcal{L}_{Z_{P}} \hat{u}^{2}=\hat{u}^{1} .
$$

The following lemma can be directly calculated using the results of section 4.1. It will be used later to extract the Levi-Civita connection one-form on $\left(\bar{N}, g_{\bar{N}}\right)$.

Lemma 3. The exterior derivatives of the one-forms in the co-frame $\left(L^{*}, v\right)$ are given by

$$
\begin{aligned}
d e^{m} & =-\sigma_{p}^{m} \wedge e^{p}, \\
d u^{1} & =0 \\
d u^{2} & =2 u^{2} \wedge u^{1}+2 \hat{u}^{2} \wedge \hat{u}^{1}+2 d_{A B} \hat{e}^{A+n} \wedge \hat{e}^{B}, \\
d \hat{e}^{m} & =\hat{e}^{m} \wedge u^{1}+e^{m} \wedge \hat{u}^{1}+\hat{u}^{2} \wedge J_{p}^{m} e^{p}-\left(\sigma_{p}^{m}+v J_{p}^{m}\right) \wedge \hat{e}^{p}+S_{p}^{m} \wedge \hat{e}^{p}, \\
d \hat{u}^{1} & =\hat{u}^{1} \wedge u^{1}-\epsilon_{1} \hat{u}^{2} \wedge v+d_{A B} e^{A} \wedge \hat{e}^{B}-\epsilon_{1} d_{A B} e^{A+n} \wedge \hat{e}^{B+n}, \\
d \hat{u}^{2} & =\hat{u}^{2} \wedge u^{1}+v \wedge \hat{u}^{1}+d_{A B} \hat{e}^{A+n} \wedge e^{B}+d_{A B} e^{A+n} \wedge \hat{e}^{B}, \\
d v & =2 d_{A B} e^{A+n} \wedge e^{B} .
\end{aligned}
$$




\begin{tabular}{|c|c|c|}
\hline & almost complex & almost para-complex. \\
\hline spatial $c$-map & $J_{1}, J_{2}, J_{3}$ & \\
temporal $c$-map & $J_{3}$ & $J_{1}, J_{2}$ \\
Euclidean $c$-map $\left(\epsilon_{1}, \epsilon_{2}\right)=(1,1)$ & $J_{1}$ & $J_{2}, J_{3}$ \\
Euclidean $c$-map $\left(\epsilon_{1}, \epsilon_{2}\right)=(1,-1)$ & $J_{2}$ & $J_{1}, J_{3}$ \\
\hline
\end{tabular}

Table 2. Properties of $J_{1}, J_{2}, J_{3}$ when restricted to ker $v$.

Be careful to note the index convention $A=1, \ldots, n$ and $m, p=1, \ldots, 2 n$. The matrix-valued one-forms $S$ and $\sigma$ were defined on $M$ in the previous subsection and are pulled-back to $P$ in the above expressions. The constant matrices $J$ and $d$ were also defined in the previous subsection. The appearance of $v$ in the expressions for $d \hat{e}^{m}, d \hat{u}^{1}, d \hat{u}^{2}$ is due to the fact that they are not invariant under the flow of $Z_{P}$.

\subsubsection{The $\varepsilon$-quaternionic structure}

We now turn our attention to the $\varepsilon$-quaternionic structure. Using the connection $v$ we may decompose the tangent space into $T P=\mathbb{R} Z_{P}+\operatorname{ker} v$ and the dual tangent space into $T^{*} P=\mathbb{R} v+Z_{P}^{0}$. The vector space $\operatorname{ker} v$ is dual to $Z_{P}^{0}$, which we recall is spanned by $L^{*}$. There exists a unique basis $L=\left(e_{m}, u_{1}, u_{2}, \hat{e}_{p}, \hat{u}_{1}, \hat{u}_{2}\right)_{m, p=1, \ldots, 2 n}$ of ker $v$ dual to $L^{*}$. In local coordinates this is given by

$$
\begin{aligned}
e_{m} & =\mathcal{T}_{m}{ }^{a} \frac{\partial}{\partial q^{a}}, & \hat{e}_{m} & =\mathcal{T}_{m}{ }^{a}\left(\frac{\partial}{\partial \hat{q}^{a}}+2 \Omega_{a b} \hat{q}^{b} \frac{\partial}{\partial \tilde{\phi}}\right), \\
u_{1} & =q^{a} \frac{\partial}{\partial q^{a}}, & \hat{u}_{1} & =-q^{a}\left(\frac{\partial}{\partial \hat{q}^{a}}+2 \Omega_{a b} \hat{q}^{b} \frac{\partial}{\partial \tilde{\phi}}\right), \\
u_{2} & =-2 H \frac{\partial}{\partial \tilde{\phi}}, & \hat{u}_{2} & =\frac{1}{2} H_{a} \Omega^{a b}\left(\frac{\partial}{\partial \hat{q}^{b}}+2 \Omega_{b c} \hat{q}^{c} \frac{\partial}{\partial \tilde{\phi}}\right) .
\end{aligned}
$$

Using this basis one may define the three endomorphisms

$$
\begin{aligned}
& J_{1}=d^{A B} e_{A} \wedge \hat{e}_{B+n}+d^{A B} \hat{e}_{A} \wedge e_{B+n}+u_{1} \wedge \hat{u}_{2}-\epsilon_{2} \hat{u}_{1} \wedge u_{2}, \\
& J_{2}=d^{A B} \hat{e}_{A} \wedge e_{B}-\epsilon_{1} d^{A B} \hat{e}_{A+n} \wedge e_{B+n}+u_{1} \wedge \hat{u}_{1}+\epsilon_{1} \epsilon_{2} u_{2} \wedge \hat{u}_{2}, \\
& J_{3}=-\epsilon_{2} d^{A B} e_{A} \wedge e_{B+n}+d^{A B} \hat{e}_{A+n} \wedge \hat{e}_{B}+u_{2} \wedge u_{1}+\hat{u}_{1} \wedge \hat{u}_{2},
\end{aligned}
$$

where we are using the notation $(X \wedge Y)(Z)=g^{\prime}(Y, Z) X-g^{\prime}(X, Z) Y$. The endomorphisms $J_{1}, J_{2}, J_{3}$ are skew-symmetric with respect to $g^{\prime}$, mutually anti-commute and satisfy

$$
\left.J_{1}^{2}\right|_{\text {ker } v}=-\epsilon_{1} \epsilon_{2},\left.\quad J_{2}^{2}\right|_{\operatorname{ker} v}=\epsilon_{2},\left.\quad J_{3}^{2}\right|_{\text {ker } v}=\epsilon_{1}, \quad J_{1} J_{2}=J_{3},
$$

which is the $\varepsilon$-quaternion algebra up to relabelling. Since the expressions for $J_{1}, J_{2}, J_{3}$ are invariant under transformations of the form (4.21) they are independent of the choice of frame $L$ of ker $v$ in the class of frames considered above and are therefore globally-defined on $P$. In table 2 we summarise which of these endomorphisms are almost complex and which are almost para-complex when restricted to ker $v \subset T P$. 
It is interesting to define two additional endomorphism fields (which are also independent of the choice of $L$ as above)

$$
\begin{aligned}
J_{3}^{\prime} & =-\epsilon_{2} d^{A B} e_{A} \wedge e_{B+n}+d^{A B} \hat{e}_{A+n} \wedge \hat{e}_{B}+u_{1} \wedge u_{2}+\hat{u}_{1} \wedge \hat{u}_{2}, \\
\tilde{J} & =-\epsilon_{2} d^{A B} e_{A+n} \wedge e_{B}+d^{A B} \hat{e}_{A+n} \wedge \hat{e}_{B}+u_{2} \wedge u_{1}+\hat{u}_{2} \wedge \hat{u}_{1},
\end{aligned}
$$

which satisfy $\left.J_{3}^{\prime 2}\right|_{\text {ker } v}=\left.\tilde{J}^{2}\right|_{\text {ker } v}=\epsilon_{1}$ and are skew-symmetric with respect to $g^{\prime}$. The previously defined endomorphism $J_{3}$ differs from $J_{3}^{\prime}$ by sign on the two-dimensional subspace spanned by $\left(u_{1}, u_{2}\right)$ and from $\tilde{J}$ by sign on the $(2 n+2)$-dimensional subspace spanned by $\left(e_{m}, \hat{u}_{1}, \hat{u}_{2}\right)$. Neither $J_{3}^{\prime}$ nor $\tilde{J}$ form part of the $\varepsilon$-quaternion algebra. Using Lemma $1, \tilde{J}$ can be written in terms of $U_{\epsilon_{1}}(1)$-invariant vectors as

$$
\begin{aligned}
\tilde{J}= & -\epsilon_{2} d^{A B} e_{A+n} \wedge e_{B} \\
& +2 e^{\phi}\left[\frac{\partial}{\partial \tilde{\phi}} \wedge \frac{\partial}{\partial \phi}+\left(\frac{\partial}{\partial \zeta^{I}}+\frac{1}{2} \tilde{\zeta}_{I} \frac{\partial}{\partial \tilde{\phi}}\right) \wedge\left(\frac{\partial}{\partial \tilde{\zeta}_{I}}-\frac{1}{2} \zeta^{I} \frac{\partial}{\partial \tilde{\phi}}\right)\right] .
\end{aligned}
$$

Here we have used the splitting of the manifold $M$ parametrised by the coordinates $q^{a}$ into the level sets of the function $\phi$ defined in (3.4). In particular, we can include $\phi$ in a new local coordinate system on $M$ consisting of $\phi$ together with a choice of local coordinates on a level set of $\phi$. The coordinates chosen on one level set are extended to the other level sets by imposing that the coordinates are invariant under the flow of $u_{1}$. In the resulting new coordinate system, one computes $u_{1}=2 \frac{\partial}{\partial \phi}$. Next, we note that

$$
\hat{\theta}_{a}:=2\left(\begin{array}{c}
\frac{\partial}{\partial \zeta^{I}}+\frac{1}{2} \tilde{\zeta}_{I} \frac{\partial}{\partial \tilde{\phi}} \\
\frac{\partial}{\partial \tilde{\zeta}_{I}}-\frac{1}{2} \zeta^{I} \frac{\partial}{\partial \tilde{\phi}}
\end{array}\right)
$$

and that

$$
d^{A B} \hat{e}_{A+n} \wedge \hat{e}_{B}=\frac{1}{2} \Omega^{m n} \hat{e}_{m} \wedge \hat{e}_{n}=\frac{1}{2} \mathcal{T}_{m}{ }^{a} \Omega^{m n} \mathcal{T}_{n}{ }^{b} \hat{\theta}_{a} \wedge \hat{\theta}_{b},
$$

where $\left(\Omega^{m n}\right):=\left(\begin{array}{cc}0 & -d \\ d & 0\end{array}\right)$. Using Lemma 1 one can then show that

$$
d^{A B} \hat{e}_{A+n} \wedge \hat{e}_{B}=\frac{1}{2} H \Omega^{a b} \hat{\theta}_{a} \wedge \hat{\theta}_{b}-\hat{u}_{2} \wedge \hat{u}_{1}
$$

from which we finally obtain (4.30). Notice that $\tilde{J}$ differs from $\pm J_{3}$ and $\pm J_{3}^{\prime}$ on the $(2 n+4)$ dimensional subspace $\operatorname{span}\left\{\partial / \partial \phi, \partial / \partial \tilde{\phi}, \partial / \partial \zeta^{I}, \partial / \partial \tilde{\zeta}_{I}\right\}=\operatorname{span}\left\{\hat{e}_{m}, u_{1}, u_{2}, \hat{u}_{1}, \hat{u}_{2}\right\}$, unless $n=0$, that is when $\bar{M}$ is a point.

The endomorphisms $J_{3}, J_{3}^{\prime}, \tilde{J}$ are invariant under the $U_{\epsilon_{1}}(1)$-action and induce almost $\epsilon_{1}$-complex structures on the $c$-map target manifold $\bar{N}$. The endomorphisms $J_{1}, J_{2}$ transform into one-another under the $U_{\epsilon_{1}}(1)$-action according to $\mathcal{L}_{Z_{P}} J_{1}=J_{2}, \mathcal{L}_{Z_{P}} J_{2}=\epsilon_{1} J_{1}$.

\section{Theorem 1.}

(a) On the target manifold of the spatial c-map the almost complex structures $J_{3}, J_{3}^{\prime}$ are integrable and $\tilde{J}$ is integrable if and only if the cubic tensor $C$ vanishes. 
(b) On the target manifold of the temporal c-map the almost complex structures $J_{3}, J_{3}^{\prime}$ are integrable and $\tilde{J}$ is integrable if and only if the cubic tensor $C$ vanishes.

(c) On the target manifold of the Euclidean c-map the almost para-complex structures $J_{3}$, $J_{3}^{\prime}$ are integrable and $\tilde{J}$ is integrable if and only if the cubic tensor $C$ vanishes.

It was shown in [64] that the almost complex structure $J_{3}$ on the target manifold of the spatial $c$-map is integrable. The other parts of this theorem will be proved on a case-by-case basis in sections 5.3-5.5. For easier reference we stated the result as three cases (a)-(c).

The endomorphisms $J_{1}, J_{2}, J_{3}$ define a (fibre-wise) $\varepsilon$-quaternionic structure $Q_{P}=$ $\operatorname{span}\left\{J_{1}, J_{2}, J_{3}\right\}$ on ker $v \subset T P$, which is skew-symmetric with respect to the metric $\left.g^{\prime}\right|_{\text {ker } v}$. Due to the transformation properties under $U_{\epsilon_{1}}(1)$, this induces an almost $\varepsilon$-quaternionic Hermitian structure $Q_{\bar{N}}$ on $\left(\bar{N}, g_{\bar{N}}\right)$. In the next section we will show, by explicit calculation, that $Q_{\bar{N}}$ is parallel with respect to the Levi-Civita connection, which proves the following theorem.

\section{Theorem 2.}

(a) The target manifold of the spatial c-map is quaternionic Kähler.

(b) The target manifold of the temporal c-map is para-quaternionic Kähler.

(c) The target manifold of the Euclidean c-map is para-quaternionic Kähler.

In all three cases the reduced scalar curvature $\nu=\operatorname{scal} /(4(n+1)(n+3))$ is equal to -2 . This can be seen by comparing the $S p_{\varepsilon}(1)$-curvature of the $c$-map target manifold

$$
R^{H}=d p+p \wedge p
$$

with the $S p_{\varepsilon}(1)$-curvature of $\varepsilon$-quaternionic projective space $\mathbb{H}_{\varepsilon} P^{n+1}$

$$
R_{0}^{H}=\frac{1}{2} \sum_{\alpha=1,2,3} J_{\alpha}^{H} \omega_{\alpha}
$$

Here the matrix $J_{\alpha}^{H}$ is given in expression (5.16) and $\omega_{\alpha}(\cdot, \cdot):=\epsilon_{\alpha} g^{\prime}\left(J_{\alpha}, \cdot\right)$ is the fundamental two-form associated with the almost $\epsilon_{\alpha}$-complex structure $J_{\alpha}$. The matrix $p$ is given for each $c$-map separately in sections 5.3-5.5. For an $\varepsilon$-quaternionic Kähler manifold the above $S p_{\varepsilon}(1)$-curvature tensors are related by [62]

$$
R^{H}=\nu R_{0}^{H}
$$

Computing both sides one finds that $\nu=-2$ in all cases.

\section{Levi-Civita connection and integrable $\varepsilon$-complex structures}

In this section we will calculate the Levi-Civita connection on the target manifold $\left(\bar{N}, g_{\bar{N}}\right)$ of the $c$-map for various spacetime signatures. We will also show that the two skew-symmetric almost $\varepsilon$-complex structures $J_{3}$ and $J_{3}^{\prime}$ introduced in the previous section are integrable. 
In order to compute the Levi-Civita connection and to check the integrability of the structures $J_{3}$ and $J_{3}^{\prime}$ one needs to calculate exterior derivatives of an appropriate local co-frame on $\bar{N}$. To do this we will make use of the partial co-frame (4.20) on the $U_{\epsilon_{1}}(1)$ principal bundle $P \rightarrow \bar{N}$. There are two complementary approaches one may take when performing these calculations:

1. Use a local section of $P \rightarrow \bar{N}$ to pull-back the partial co-frame from $P$ to a co-frame of $\bar{N}$ and then perform calculations.

2. Perform calculations directly on $P$ using the partial co-frame and then use a local section to pull-back the results to $\bar{N}$.

We will adopt approach 2 since one only needs to make a choice of local section after all calculations have been performed. There is a slight complication due to the fact that the partial co-frame (4.20) is not invariant under the flow of the fundamental vector field $Z_{P}$ and therefore not projectable to $\bar{N}$, which we address in section 5.1. The relation between the two approaches is discussed in section 5.2. The explicit calculation of the Levi-Civita connection and integrability of the $\varepsilon$-complex structures for various spacetime signatures are presented case-by-case in the remaining sections.

\subsection{Calculating on $P$ using a non-invariant partial co-frame}

In this section we want to discuss the following problem. Let $(M, g)$ be a pseudoRiemannian manifold with almost $\varepsilon$-quaternionic Hermitian structure $Q$ and $\pi: P \rightarrow M$ be a principal bundle with structure group $G$. Suppose we are given pointwise linearly independent one-forms $\theta^{i}, i=1, \ldots, n=\operatorname{dim}(M)$ on some open subset $U \subset P$, which are horizontal in the sense that they vanish on any vertical vector, such that

$$
\pi^{*} g=\eta_{i j} \theta^{i} \theta^{j}
$$

where $\left(\eta_{i j}\right)=\operatorname{diag}\left(\mathbb{1}_{k},-\mathbb{1}_{\ell}\right)$ is the Gram matrix of an orthonormal frame in standard ordering. We will assume that $\pi^{*} T M$ is trivial on $U$. Systems $\left(\theta^{i}\right)$ as above will be called partial co-frames of $P$ over $U$. Notice that given a principal connection on $P$ and a basis of $\mathfrak{g}=$ Lie $G$, any partial co-frame of $P$ over $U$ is canonically extended to a co-frame of $P$ over $U$.

The problem is to show that $Q$ is parallel with respect to the Levi-Civita connection, and, hence, the manifold $(M, g)$ is $\varepsilon$-quaternionic Kähler. This involves computing the LeviCivita connection of $g$ in terms of $\left(\theta^{i}\right)$, without assuming that the forms $\theta^{i}$ are $G$-invariant and, hence, projectable to $M$.

Proposition 3. Under the above assumptions, the system of equations

$$
d \theta^{i}+\sigma_{j}^{i} \wedge \theta^{j}=0
$$

has a unique solution $\sigma=\left(\sigma_{j}^{i}\right) \in \Omega^{1}(U, \mathfrak{s o}(k, \ell))$. Given a second system of $n$ linearly independent horizontal one-forms $\left(\tilde{\theta}^{i}\right)$ on $U \subset P$, the solution $\tilde{\sigma}=\left(\tilde{\sigma}_{j}^{i}\right)$ of the system

$$
d \tilde{\theta}^{i}+\tilde{\sigma}_{j}^{i} \wedge \tilde{\theta}^{j}=0
$$


is related to $\sigma$ by

$$
\tilde{\sigma}=-(d A) A^{-1}+A \sigma A^{-1},
$$

where $A=\left(A_{j}^{i}\right) \in C^{\infty}(U, O(k, \ell))$ is the gauge transformation relating $\left(\theta^{i}\right)$ with $\left(\tilde{\theta}^{i}\right)$, that is $\tilde{\theta}^{i}=A_{j}^{i} \theta^{j}$.

Proof. We first prove the uniqueness. Suppose that $\sigma^{\prime}$ is a second solution of (5.1). Then the difference $\Delta=\left(\Delta_{j}^{i}\right)=\sigma^{\prime}-\sigma \in \Omega^{1}(U, \mathfrak{s o}(k, \ell))$ satisfies the equations $\Delta_{j}^{i} \wedge \theta^{j}=0$. For the coefficients $\Delta_{j k}^{i}$ in the expansion $\Delta_{k}^{i}=\Delta_{j k}^{i} \theta^{j}$ this implies $\Delta_{j k}^{i}=\Delta_{k j}^{i}$. Therefore $\Delta_{j i k}:=\eta_{i l} \Delta_{j k}^{l}$ is antisymmetric in $(i, k)$ and symmetric in $(j, k)$, which implies $\Delta=0$.

One can easily check that given a solution $\sigma$ of (5.1) and a gauge transformation $A \in C^{\infty}(U, O(k, \ell)), \tilde{\sigma}=-(d A) A^{-1}+A \tilde{\sigma} A^{-1}$ is a solution of (5.2), if we define $\tilde{\theta}^{i}=A_{j}^{i} \theta^{j}$.

Now we prove the existence. Given the above hypothesis on $U$, we can assume without restriction of generality that $U=\pi^{-1}\left(U_{0}\right)$ is the preimage of an open subset $U_{0} \subset M$ on which an orthonormal co-frame $\left(\theta_{0}^{i}\right)$ exists. It is sufficient to remark that the pullback of the connection one-form $\sigma_{0}$ of the Levi-Civita connection of $(M, g)$ with respect to the co-frame $\left(\theta_{0}^{i}\right)$ gives a solution of (5.1), where $\left(\theta^{i}\right)=\left(\pi^{*} \theta_{0}^{i}\right)$. The equation (5.1) is in fact obtained as the pullback of the equation $d \theta_{0}+\sigma_{0} \wedge \theta_{0}=0$, which expresses the vanishing of the torsion of the Levi-Civita connection of $(M, g)$. Here $\theta_{0}$ is the column vector with entries $\theta_{0}^{i}$.

The almost $\varepsilon$-quaternionic Hermitian structure $Q$ on $M$ induces a (fibre-wise) $\varepsilon$ quaternionic Hermitian structure $Q_{P}$ in the normal bundle $\mathcal{N}=T P / T^{v} P$ to the fibres of $P \rightarrow M$, where $T^{v} P \subset T P$ denotes the vertical distribution. The $\varepsilon$-quaternionic structure $Q_{P}$ is Hermitian in the sense that it consists of endomorphisms which are skew-symmetric with respect to the (fibre-wise) metric $\pi^{*} g$ in $\mathcal{N}$. By construction $Q_{P}$ is invariant under the $G$-action on $\mathcal{N}$ induced by the principal $G$-action on $P$. Conversely, a fibre-wise skew-symmetric $\varepsilon$-quaternionic structure $Q_{P}$ on $\left(\mathcal{N}, \pi^{*} g\right)$, which is invariant under the $G$ action on $\mathcal{N}$, induces an almost $\varepsilon$-quaternionic Hermitian structure $Q$ on $M$, which may be parallel or not.

Proposition 4. Given a G-invariant skew-symmetric fibre-wise $\varepsilon$-quaternionic structure $Q_{P}$ on $\mathcal{N}$ the induced almost $\varepsilon$-quaternionic Hermitian structure $Q$ on $(M, g)$ is parallel with respect to the Levi-Civita connection if the solution of (5.1) takes values in the Lie algebra $\mathfrak{s p}_{\varepsilon}(1) \oplus \mathfrak{s p}_{\varepsilon}(k, \ell)$, provided the partial co-frame $\left(\theta^{i}\right)$ is $\varepsilon$-quaternionic.

Proof. Consider an open subset $U \subset P$ on which an $\varepsilon$-quaternionic partial co-frame $\left(\theta^{i}\right)$ is defined. We may assume without restriction of generality that $U=\pi^{-1}\left(U_{0}\right)$ is the preimage of an open subset $U_{0} \subset M$ on which an $\varepsilon$-quaternionic co-frame $\left(\theta_{0}^{i}\right)$ exists. This may be pulled back to give another $\varepsilon$-quaternionic partial co-frame $\left(\tilde{\theta}^{i}\right)=\left(\pi^{*} \theta_{0}^{i}\right)$. Since both $\left(\theta^{i}\right)$ and $\left(\tilde{\theta}^{i}\right)$ are $\varepsilon$-quaternionic partial co-frames they are related to one-another by a gauge transformation of the form $A=\left(A_{j}^{i}\right) \in C^{\infty}\left(U, S p_{\varepsilon}(1) \cdot S p_{\varepsilon}(k, \ell)\right)$. Let us denote by $\sigma$ the solution of (5.1) in the basis $\left(\theta^{i}\right)$ and by $\tilde{\sigma}$ the solution in the basis $\left(\tilde{\theta}^{i}\right)$.

Suppose that $\sigma$ takes values in $\mathfrak{s p}_{\varepsilon}(1) \cdot \mathfrak{s p}_{\varepsilon}(k, \ell)$. From (5.3) it follows that $\tilde{\sigma}$ also takes values in $\mathfrak{s p}_{\varepsilon}(1) \cdot \mathfrak{s p}_{\varepsilon}(k, \ell)$. Since $\tilde{\sigma}$ is the pull-back of the Levi-Civita connection one-form in an $\varepsilon$-quaternionic co-frame it follows that the Levi-Civita connection on $M$ takes values in $\mathfrak{s p}_{\varepsilon}(1) \cdot \mathfrak{s p}_{\varepsilon}(k, \ell)$ when written in an $\varepsilon$-quaternionic co-frame. 
Proposition 3 shows that in order to compute the Levi-Civita connection of a manifold $\left(\bar{N}, g_{\bar{N}}\right)$ in the image of the $c$-map it is sufficient to solve the equation (5.1) locally on $P$ without having to assume that the partial co-frame $\left(\theta^{i}\right)$ is projectable. If the solution to (5.1) takes values in $\mathfrak{s p}_{\varepsilon}(1) \oplus \mathfrak{s p}_{\varepsilon}(k, \ell)$ in an $\varepsilon$-quaternionic partial co-frame then the manifold $\left(\bar{N}, g_{\bar{N}}\right)$ is $\varepsilon$-quaternionic Kähler by Proposition 4 .

\subsection{Alternative approach: calculating on $\bar{N}$ using a co-frame}

Let us now briefly discuss an alternative way of calculating exterior derivatives and the Levi-Civita connection directly on the target manifold $\left(\bar{N}, g_{\bar{N}}\right)$ in the image of the $c$-map.

Let $U \subset P$ be an open set on which the partial co-frame (4.20) is defined. Consider any local section $s: U_{0} \rightarrow P$ with values in $U$, for example the local section defined by the equation $x^{0}=0$. (Recall that $x^{0}=q^{0}$ is one of the functions $\left(q^{a}, \hat{q}^{b}, \tilde{\phi}\right)$ on $P$ introduced in section 4.2.) We may use the section $s$ to define a co-frame on $U_{0} \subset \bar{N}$ given by

$$
\left(e_{0}^{m}, u_{0}^{1}, u_{0}^{2}, \hat{e}_{0}^{m}, \hat{u}_{0}^{1}, \hat{u}_{0}^{2}\right)=s^{*}\left(e^{m}, u^{1}, u^{2}, \hat{e}^{m}, \hat{u}^{1}, \hat{u}^{2}\right) .
$$

It is then possible to calculate the exterior derivatives and the Levi-Civita connection in this local co-frame on $\bar{N}$.

One may relate this approach to that of section 5.1 as follows. Since the exterior derivative commutes with the pull-back of a differentiable map we have

$$
d e_{0}^{m}=s^{*} d e^{m}, \quad d u_{0}^{1}=s^{*} d u^{1}, \quad \text { etc. }
$$

where the exact expressions on the r.h.s. can be read off from (4.24). Moreover, from Proposition 3 it follows that the Levi-Civita connection $\sigma_{0}$ on $\bar{N}$ in the basis (5.4) is given by the pull-back of the unique solution $\sigma$ of equation (5.1) in the basis (4.20), which is calculated in the following sections.

\subsection{The spatial $c$-map}

In this section we consider the reduction over space from $3+1$ to $2+1$ dimensions. This means that one must set $\epsilon_{1}=-1$ and $\epsilon_{2}=-1$ in the expressions in section 4.2. Recall from section 3.2 that the Hesse potential $H$ is assumed to be negative.

In order to expose the quaternionic geometry we define the complex partial coframe on $P$

$$
\begin{aligned}
\mathbf{u} & =i e^{-\phi / 2}\left(X^{I} d \tilde{\zeta}_{I}-F_{I}(X) d \zeta^{I}\right), \\
\mathbf{v} & =e^{-\phi}\left[\frac{1}{2} d e^{\phi}+i\left(d \tilde{\phi}+\frac{1}{2}\left(\zeta^{I} d \tilde{\zeta}_{I}-\tilde{\zeta}_{I} d \zeta^{I}\right)\right)\right], \\
\mathbf{e}^{A} & =P_{I}^{A} d X^{I} \\
\mathbf{E}^{A} & =-i e^{-\phi / 2} P_{I}^{A} N^{I J}\left(d \tilde{\zeta}_{J}-\mathcal{N}_{J K} d \zeta^{K}\right) .
\end{aligned}
$$

Recall that $X^{I}=e^{-\phi / 2} Y^{I}$, and, due to homogeneity, $N_{I J}(X, \bar{X})=N_{I J}(Y, \bar{Y})$ and $\mathcal{N}_{I J}(X, \bar{X})=\mathcal{N}_{I J}(Y, \bar{Y})$. We have locally defined the matrix $\left(P_{I}{ }^{A}\right)$ with entries

$$
P_{I}{ }^{A}=e^{\phi / 2}\left(\mathcal{P}_{a}{ }^{A}+i \mathcal{P}_{a}{ }^{A+n}\right) \Pi_{I}{ }^{a},
$$


where $\Pi_{I}^{a}$ represents the holomorphic projection from the special holomorphic coordinates $Y^{I}$ to special real coordinates $q^{a}$ :

$$
d q^{a}=\Pi_{I}^{a} d Y^{I}+\bar{\Pi}_{I}^{a} d \bar{Y}^{I}, \quad\left(\Pi_{I}^{a}\right)=\frac{\partial q^{a}}{\partial Y^{I}}=\left(\frac{1}{2}\left(\delta_{I}^{J}\right), \frac{1}{2}\left(F_{I J}\right)\right) .
$$

Notice that $P_{I}^{A} Y^{I}=0$, and, hence, $P_{I}{ }^{A} d X^{I}=e^{-\phi / 2} P_{I}{ }^{A} d Y^{I}$. Using the local section $s=\left\{\operatorname{Im}\left(X^{0}\right)=0\right\}\left(=\left\{x^{0}=0\right\}\right)$ of $P \rightarrow \bar{N}$ discussed in section 5.2 one can pull-back (5.5) to the complex orthonormal co-frame on $\left(\bar{N}, g_{\bar{N}}\right)$ presented in $[25] .{ }^{10}$

Proposition 5. The complex partial co-frame (5.5) is related to the real partial coframe (4.20) introduced in section 4.2 by

$$
\begin{aligned}
\mathbf{u} & =\hat{u}^{1}+i \hat{u}^{2}, & \mathbf{e}^{A} & =e^{A}+i e^{A+n}, \\
\mathbf{v} & =u^{1}+i u^{2}, & \mathbf{E}^{A} & =\hat{e}^{A}+i \hat{e}^{A+n} .
\end{aligned}
$$

The one-form $v$ may be written as

$$
v=\frac{1}{2 i}\left(X^{I} N_{I J} d \bar{X}^{J}-d X^{I} N_{I J} \bar{X}^{J}\right)
$$

Proof. Using $e^{\phi}=-2 H$ and

$$
Y^{I}=x^{I}+i u^{I}, \quad F_{I}=y_{I}+i v_{I}, \quad H_{a}=\left(2 v_{I},-2 u^{I}\right)^{T},
$$

the first two expressions are calculated to be

$$
\begin{aligned}
& \mathbf{u}=-i \frac{1}{2 H}\left[x^{I} d \tilde{\zeta}_{I}-y_{I} d \zeta^{I}+i\left(u^{I} d \tilde{\zeta}_{I}-v_{I} d \zeta^{I}\right)\right]=-i \frac{1}{2 H}\left[2 q^{a} \Omega_{a b} d \hat{q}^{b}-i\left(H_{a} d \hat{q}^{a}\right)\right] \\
& \mathbf{v}=-\frac{1}{2 H}\left[-d H+i\left(d \tilde{\phi}+2 \hat{q}^{a} \Omega_{a b} d \hat{q}^{b}\right)\right]
\end{aligned}
$$

Comparing with the explicit expressions in (4.20) gives the desired result. Next, we observe that

$$
\begin{aligned}
e^{A}+i e^{A+n} & =\frac{1}{2}\left(I d-i J^{*}\right)\left(e^{A}+i e^{A+n}\right)=\left(\mathcal{P}_{a}{ }^{A}+i \mathcal{P}_{a}{ }^{A+n}\right) \Pi_{I}{ }^{a} d Y^{I} \\
& =e^{-\phi / 2} P_{I}^{A} d Y^{I}=P_{I}^{A} d X^{I}=\mathbf{e}^{A} .
\end{aligned}
$$

Using the fact $P_{I}{ }^{A} Y^{I}=0$ along with (5.7) and (5.6) one may write

$$
\begin{aligned}
\mathbf{E}^{A} & =-i e^{-\phi / 2} P_{I}{ }^{A} N^{I J}\left(d \tilde{\zeta}_{J}-\bar{F}_{J K} d \zeta^{K}\right)=-4 i e^{-\phi / 2} P_{I}{ }^{A} N^{I J} \bar{\Pi}_{J}^{b} \Omega_{b c} d \hat{q}^{c} \\
& =-4 i\left(\mathcal{P}_{a}{ }^{A}+i \mathcal{P}_{a}{ }^{A+n}\right) \Pi_{I}{ }^{a} N^{I J} \bar{\Pi}_{J}^{b} \Omega_{b c} d \hat{q}^{c} .
\end{aligned}
$$

Making use of the identity $4 \Pi_{I}^{a} N^{I J} \bar{\Pi}_{J}^{b}=H^{a b}+\frac{i}{2} \Omega^{a b}$, which can be easily verified using (2.11) and (5.7), along with the expression for $J^{*}{ }_{b}^{a}$ given by the components of (2.9), we can write

$$
\mathbf{E}^{A}=-\frac{i}{2}\left(\mathcal{P}_{a}{ }^{A}+i \mathcal{P}_{a}{ }^{A+n}\right)\left(J^{*}{ }_{b}{ }^{a} d \hat{q}^{b}+i d \hat{q}^{a}\right)
$$

\footnotetext{
${ }^{10}$ Recall that we use the D-gauge $-N_{I J} X^{I} \bar{X}^{J}=1$ in order to fix the scale transformations of the CASK manifold $M$, whereas in [25] they are fixed by setting $\left|X^{0}\right|=1$. These two choices are related by the transformation $X^{I} \mapsto \frac{1}{\left|X^{0}\right|} X^{I}$.
} 
From (4.8) it follows that $\mathcal{P}_{b}{ }^{m} J^{*}{ }_{a}^{b}=J^{*}{ }_{p}{ }^{m} \mathcal{P}_{a}{ }^{p}$, and, hence, $\mathbf{E}^{A}=\hat{e}^{A}+i \hat{e}^{A+n}$. Lastly, we calculate

$$
\begin{aligned}
\frac{1}{2 i} & \left(X^{I} N_{I J} d \bar{X}^{J}-d X^{I} N_{I J} \bar{X}^{J}\right) \\
& =e^{-\phi} \operatorname{Im}\left(Y^{I} N_{I J}(Y, \bar{Y}) d \bar{Y}^{J}\right)=-e^{-\phi} \operatorname{Re}\left(F_{I} d \bar{Y}^{I}-Y^{I} d \bar{F}_{I}\right) \\
& =\frac{1}{2 H}\left(y_{I} d x^{I}-x^{I} d y_{I}-v_{I} d u^{I}+u^{I} d v_{I}\right)=-\frac{1}{2 H}\left(q^{a} \Omega_{a b} d q^{b}-\frac{1}{4} H_{a} \Omega^{a b} d H_{b}\right) \\
& =-\frac{1}{H} q^{a} \Omega_{a b} d q^{b}=v
\end{aligned}
$$

where in the second line we used (2.7) and in last line (2.10).

The exterior derivatives of the one-forms in the complex co-frame may be written as [25] (see also [69] for the indefinite case)

$$
\begin{aligned}
d \mathbf{u} & =\left(-u^{1}+i v\right) \wedge \mathbf{u}-d_{A B} \overline{\mathbf{E}}^{A} \wedge \mathbf{e}^{B}, \\
d \mathbf{v} & =\mathbf{u} \wedge \overline{\mathbf{u}}+\mathbf{v} \wedge \overline{\mathbf{v}}+d_{A B} \mathbf{E}^{A} \wedge \overline{\mathbf{E}}^{B}, \\
d \mathbf{e}^{A} & =-\sigma^{\mathbb{C} A}{ }_{B} \wedge \mathbf{e}^{B}, \\
d \mathbf{E}^{A} & =\left(-u^{1}-i v\right) \wedge \mathbf{E}^{A}-\overline{\mathbf{u}} \wedge \mathbf{e}^{A}-\sigma^{\mathbb{C} A}{ }_{B} \wedge \mathbf{E}^{B}+S_{B C}^{\mathbb{C} A}{ }_{B C} \overline{\mathbf{e}}^{B} \wedge \overline{\mathbf{E}}^{C},
\end{aligned}
$$

where $\sigma_{B}^{\mathbb{C A}}:=\sigma_{B}^{A}+i \sigma_{B}^{A+n}$ and $S_{B C}^{\mathbb{C A}}:=S_{B C}^{A}+i S_{B C}^{A+n}$. These expressions may be checked using (4.24) and the identities (4.13) and (4.17).

Proof of Theorem 1 (a). The following proof that $J_{3}$ is integrable was provided in [64]: a basis of the $+i$ eigendistribution of $J_{3}^{*}$ is given by $\mathcal{B}^{(1,0)}=\left(\overline{\mathbf{u}}, \mathbf{v}, \overline{\mathbf{e}}^{A}, \mathbf{E}^{A}\right)$. Each term in the exterior derivative of any element in $\mathcal{B}^{(1,0)}$ contains a one-form in the set $\mathcal{B}^{(1,0)}$. Therefore the distribution is integrable by the Newlander-Nirenberg theorem, hence the almost-complex structure $J_{3}$ is integrable.

We now consider the integrability of $J_{3}^{\prime}$ and $\tilde{J}$. A basis of the $+i$ eigendistribution of $J_{3}^{\prime *}$ is given by $\mathcal{B}^{\prime(1,0)}=\left(\overline{\mathbf{u}}, \overline{\mathbf{v}}, \overline{\mathbf{e}}^{A}, \mathbf{E}^{A}\right)$, and by the same argument as above $J_{3}^{\prime}$ is integrable. A basis of the $+i$ eigendistribution of $\tilde{J}$ is given by $\left(\mathbf{u}, \mathbf{v}, \mathbf{e}^{A}, \mathbf{E}^{A}\right)$, and therefore $\tilde{J}$ is integrable if and only if $S_{B C}^{A}=S_{B C}^{A+n}=0$, which is the case if and only if the cubic tensor $C$, defined in (4.9), vanishes. This is true if and only if the holomorphic prepotential $F$, or, equivalently, the Hesse potential $H$ on the corresponding CASK manifold $M$ is a quadratic polynomial.

Proof of Theorem 2 (a). The complex one-forms, along with their conjugates, may be gathered together into the quaternionic vielbein

$$
\mathcal{U}^{\mathcal{A} \mu}=\frac{1}{\sqrt{2}}\left(\begin{array}{cccc}
\mathbf{u} & \mathbf{e}^{A} & -\overline{\mathbf{v}} & -\overline{\mathbf{E}}^{A} \\
\mathbf{v} & \mathbf{E}^{A} & \overline{\mathbf{u}} & \overline{\mathbf{e}}^{A}
\end{array}\right)
$$

In this co-frame the Levi-Civita connection one-form decomposes according to (2.21), where 
$p, q, t$ are given by $[25]$

$$
\begin{aligned}
& p=\left(\begin{array}{cc}
\frac{i}{2} u^{2}-\frac{i}{2} v-\hat{u}^{1}-i \hat{u}^{2} \\
\hat{u}^{1}-i \hat{u}^{2}-\frac{i}{2} u^{2}+\frac{i}{2} v
\end{array}\right) \\
& q=\left(\begin{array}{cc}
-\frac{3 i}{2} u^{2}-\frac{i}{2} v & \left(\hat{e}^{C}-i \hat{e}^{C+n}\right) d_{C B} \\
-\hat{e}^{A}-i \hat{e}^{A+n} & \sigma_{B}^{A}+i \sigma_{B}^{A+n}-\frac{i}{2}\left(u^{2}-v\right) \delta_{B}^{A}
\end{array}\right), \\
& t=\left(\begin{array}{cc}
0 & 0 \\
0 & \left(S_{B C}^{A}+i S_{B C}^{A+n}\right)\left(\hat{e}^{C}-i \hat{e}^{C+n}\right)
\end{array}\right) .
\end{aligned}
$$

The quaternionic structure is therefore parallel with respect to the Levi-Civita connection.

Let us briefly explain how one may check that the above expression for the Levi-Civita connection is correct. It is obvious from the formalism that the above expression defines a metric connection so it suffices to check that its torsion is zero. In terms of an $\varepsilon$-quaternionic vielbein the latter condition is given by

$$
d \mathcal{U}^{\mathcal{A} \mu}+\Omega^{\mathcal{A} \mu}{ }_{\mathcal{B} \nu} \wedge \mathcal{U}^{\mathcal{B} \nu}=0
$$

This can be naturally split into two separate sets of equations

$$
\begin{aligned}
d e^{\mathcal{A} I} & =-p^{\mathcal{A}}{ }_{\mathcal{B}} e^{\mathcal{B} I}-q^{I}{ }_{J} e^{\mathcal{A} J}-t^{I}{ }_{J} f^{\mathcal{A} J}, \\
d f^{\mathcal{A} I} & =-p^{\mathcal{A}}{ }_{\mathcal{B}} f^{\mathcal{B} I}+s^{I}{ }_{J} e^{\mathcal{A} J}+\left(\eta^{\prime} q^{T} \eta^{\prime}\right)^{I}{ }_{J} f^{\mathcal{A} J},
\end{aligned}
$$

where we have defined

$$
e^{\mathcal{A} I}:=\left(\begin{array}{cc}
\mathbf{u} & \mathbf{e}^{A} \\
\mathbf{v} & \mathbf{E}^{A}
\end{array}\right), \quad f^{\mathcal{A} I}:=\left(\begin{array}{cc}
-\overline{\mathbf{v}} & -\overline{\mathbf{E}}^{A} \\
\overline{\mathbf{u}} & \overline{\mathbf{e}}^{A}
\end{array}\right), \quad \eta^{\prime}:=\left(\begin{array}{ll}
1 & 0 \\
0 & d
\end{array}\right) .
$$

In the quaternionic case $s=\bar{t}$ and $f^{\mathcal{A} I}=\epsilon^{\mathcal{A B}} \bar{e}^{\mathcal{B} J}$, and therefore the second set of equations follows from the first set by complex conjugation. However in the para-quaternionic case, which we will deal with in the following sections, the second set of equations are not implied by the first, and must be checked independently.

Let us end by explicitly checking, for instance, that the formula for $d \mathbf{E}^{A}$ obtained from (5.11) coincides with the exterior derivative of $\mathbf{E}^{A}$ as given in (5.9):

$$
\begin{aligned}
d \mathbf{E}^{A}= & -p^{1}{ }_{0} \mathbf{e}^{A}-p^{1}{ }_{1} \mathbf{E}^{A}-q^{A}{ }_{0} \mathbf{v}-q^{A}{ }_{B} \mathbf{E}^{B}-t^{A}{ }_{0} \overline{\mathbf{u}}-t^{A}{ }_{B} \overline{\mathbf{e}}^{A} \\
= & \hat{e}^{A} u^{1}+i \hat{e}^{A+n} u^{1}+e^{A} \hat{u}^{1}+i e^{A+n} \hat{u}^{1}-\hat{u}^{2} e^{A+n}+i \hat{u}^{2} e^{A}+v \hat{e}^{A+n}-i v \hat{e}^{A} \\
& -\left(\sigma^{A}{ }_{B}+i \sigma^{A+n}{ }_{B}\right)\left(\hat{e}^{B}+i \hat{e}^{B+n}\right)-\left(S^{A}{ }_{B C}+i S^{A+n}{ }_{B C}\right)\left(\hat{e}^{C}-i \hat{e}^{C+n}\right)\left(e^{B}-i e^{B+n}\right) \\
= & \left(-u^{1}-i v\right)\left(\hat{e}^{A}+i \hat{e}^{A+n}\right)-\left(\hat{u}^{1}-i \hat{u}^{2}\right)\left(e^{A}+i e^{A+n}\right) \\
& -\left(\sigma_{B}^{A}{ }_{B}+i \sigma^{A+n}{ }_{B}\right)\left(\hat{e}^{B}+i \hat{e}^{B+n}\right)+\left(S_{B C}^{A}+i S_{B C}^{A+n}{ }_{B C}\right)\left(e^{B}-i e^{B+n}\right)\left(\hat{e}^{C}-i \hat{e}^{C+n}\right) .
\end{aligned}
$$

Here we have omitted writing the symbol for the wedge product. 


\subsection{The temporal $c$-map}

We now consider the reduction over time from $3+1$ to $3+0$ dimensions. In this case we must set $\epsilon_{1}=-1$ and $\epsilon_{2}=1$. Recall that in our construction of the $c$-map the spatial and temporal $c$-map have the same target manifold but different metrics. In particular, we may use the same partial co-frame $L^{*}$ on $P$ defined by (4.20) in both cases.

Proof of Theorem 1 (b). It follows from (4.22) and (4.26) that the almost-complex structures $J_{3}$ and $J_{3}^{\prime}$ in the case $\left(\epsilon_{1}, \epsilon_{2}\right)=(-1,1)$ coincide with $-J_{3}^{\prime}$ and $-J_{3}$, respectively, in the case $\left(\epsilon_{1}, \epsilon_{2}\right)=(-1,-1)$. Therefore the integrability of these structures follows from the proof of Theorem 1 (a) given in section 5.3.

The almost-complex structure $\tilde{J}$ in the case $\left(\epsilon_{1}, \epsilon_{2}\right)=(-1,1)$ coincides with $-\tilde{J}$ in the case $\left(\epsilon_{1}, \epsilon_{2}\right)=(-1,-1)$ except for its action on the two-dimensional subspace spanned by $\left(u_{1}, u_{2}\right)$, where it acts with opposite sign. Taking this into account, one may use the same argument as in the proof of Theorem 1 (a) that $\tilde{J}$ is integrable if and only if $C=0$.

Let us define the real partial co-frame

$$
\begin{aligned}
\mathbf{u} & =u^{2}-\hat{u}^{1}, & \tilde{\mathbf{u}} & =u^{2}+\hat{u}^{1}, \\
\mathbf{v} & =u^{1}+\hat{u}^{2}, & \tilde{\mathbf{v}} & =u^{1}-\hat{u}^{2}, \\
\mathbf{e}^{A} & =-e^{A+n}-\hat{e}^{A}, & \tilde{\mathbf{e}}^{A} & =-e^{A+n}+\hat{e}^{A}, \\
\mathbf{E}^{A} & =-e^{A}-\hat{e}^{A+n}, & \tilde{\mathbf{E}}^{A} & =-e^{A}+\hat{e}^{A+n},
\end{aligned}
$$

which we gather together into the para-quaternionic vielbein

$$
\mathcal{U}^{\mathcal{A} \mu}=\frac{1}{\sqrt{2}}\left(\begin{array}{cccc}
\mathbf{u} & \mathbf{e}^{A} & -\tilde{\mathbf{v}} & -\tilde{\mathbf{E}}^{A} \\
\mathbf{v} & \mathbf{E}^{A} & \tilde{\mathbf{u}} & \tilde{\mathbf{e}}^{A}
\end{array}\right) .
$$

One may use Proposition 5 to write the vielbein in terms of the real and imaginary parts of the complex co-frame (5.5). Notice that the above expression for the para-quaternionic vielbein is not related to the expression for the spatial $c$-map quaternionic vielbein by replacing complex coordinates with the para-complex coordinates. However, as we will explain in the next section, such a relationship does exist for the vielbeins of the spatial and Euclidean $c$-maps.

Proof of Theorem $2(b)$. In the frame (5.12) the Levi-Civita connection one-form decomposes according to (2.21), where $p, q, t, s$ are given by

$$
\begin{aligned}
& p=\left(\begin{array}{cc}
\hat{u}^{2} & -\frac{1}{2}\left(u^{2}+v\right)+\hat{u}^{1} \\
\frac{1}{2}\left(u^{2}+v\right)+\hat{u}^{1} & -\hat{u}^{2}
\end{array}\right), \\
& q=\left(\begin{array}{cc}
0 & \hat{e}^{C+n} d_{C B} \\
\hat{e}^{A+n} & \sigma_{B}^{A}-S_{B m}^{A+n} \hat{e}^{m}
\end{array}\right), \\
& t=\left(\begin{array}{c}
\frac{3}{2} u^{2}-\frac{1}{2} v \\
-\hat{e}^{A}-\sigma_{B}^{A+n}+S_{B m}^{A}{ }_{B m} \hat{e}^{m}-\frac{1}{2}\left(u^{2}+v\right) \delta_{B}^{A}
\end{array}\right), \\
& s=\left(\begin{array}{cc}
\frac{3}{2} u^{2}-\frac{1}{2} v & \hat{e}^{C} d_{C B} \\
\hat{e}^{A} & -\sigma_{B}^{A+n}{ }_{B}-S_{B m}^{A} \hat{e}^{m}-\frac{1}{2}\left(u^{2}+v\right) \delta_{B}^{A}
\end{array}\right) .
\end{aligned}
$$


The para-quaternionic structure is therefore parallel with respect to the Levi-Civita connection.

\subsection{The Euclidean $c$-map}

We now consider the reduction from $4+0$ to $3+0$ dimensions. In this case we make the choice $\epsilon_{1}=1$ but $\epsilon_{2}$ may be left arbitrary.

Let us define the real partial co-frame on $P$

$$
\begin{aligned}
\mathbf{u} & =\hat{u}^{1}-\epsilon_{2} \hat{u}^{2}, & \tilde{\mathbf{u}} & =-\epsilon_{2} \hat{u}^{1}-\hat{u}^{2}, \\
\mathbf{v} & =u^{1}+u^{2}, & \tilde{\mathbf{v}} & =u^{1}-u^{2}, \\
\mathbf{e}^{A} & =e^{A}-\epsilon_{2} e^{A+n}, & \tilde{\mathbf{e}}^{A} & =e^{A}+\epsilon_{2} e^{A+n}, \\
\mathbf{E}^{A} & =-\epsilon_{2} \hat{e}^{A}+\hat{e}^{A+n}, & \tilde{\mathbf{E}}^{A} & =\hat{e}^{A}+\epsilon_{2} \hat{e}^{A+n} .
\end{aligned}
$$

Proposition 6. The following para-complex partial co-frame (and its para-complex conjugate) is related to the above real partial co-frame by replacing the para-complex unit $i_{\epsilon_{1}}$ with 1:

$$
\begin{aligned}
\hat{u}^{1}+i_{\epsilon_{1}} \hat{u}^{2} & =i_{\epsilon_{1}} e^{-\phi / 2}\left(X^{I} d \tilde{\zeta}_{I}-F_{I} d \zeta^{I}\right), \\
u^{1}+i_{\epsilon_{1}} u^{2} & =e^{-\phi}\left[\frac{1}{2} d e^{\phi}+i_{\epsilon_{1}}\left(d \tilde{\phi}+\frac{1}{2}\left(\zeta^{I} d \tilde{\zeta}_{I}-\tilde{\zeta}_{I} d \zeta^{I}\right)\right)\right], \\
e^{A}+i_{\epsilon_{1}} e^{A+n} & =P_{I}{ }^{A} d X^{I}, \\
\hat{e}^{A}+i_{\epsilon_{1}} \hat{e}^{A+n} & =-i_{\epsilon_{1}} e^{-\phi / 2} P_{I}^{A} N^{I J}\left(d \tilde{\zeta}_{J}-\bar{F}_{J K} d \zeta^{K}\right),
\end{aligned}
$$

where $d q^{a}=: \Pi_{I}{ }^{a} d Y^{I}+\bar{\Pi}_{I}^{a} d \bar{Y}^{I}$ and $P_{I}{ }^{A}:=e^{\phi / 2}\left(\mathcal{P}_{a}{ }^{A}+i_{\epsilon_{1}} \mathcal{P}_{a}{ }^{A+n}\right) \Pi_{I}{ }^{a}$. The one-form $v$ may be written as

$$
v=\frac{1}{2 i_{\epsilon_{1}}}\left(X^{I} N_{I J} d \bar{X}^{J}-d X^{I} N_{I J} \bar{X}^{J}\right)
$$

Proof. The proof is analogous to the proof of Proposition 5. In the para-complex case one must use the identities $\mathbf{e}^{A}=\frac{1}{2}\left(I d+\epsilon_{1} i_{\epsilon_{1}} J^{*}\right) \mathbf{e}^{A}$ and $4 \Pi_{I}^{a} N^{I J} \bar{\Pi}_{J}^{b}=H^{a b}-\epsilon_{1} \frac{i_{\epsilon_{1}}}{2} \Omega^{a b}$.

The exterior derivatives of the one-forms in the real partial co-frame can be computed from Lemma 3

$$
\begin{aligned}
d \mathbf{u}= & \left(-u^{1}-\epsilon_{2} v\right) \wedge \mathbf{u}-\delta_{A B} \tilde{\mathbf{E}}^{A} \wedge \mathbf{e}^{B}, \\
d \mathbf{v}= & \mathbf{u} \wedge \tilde{\mathbf{u}}+\mathbf{v} \wedge \tilde{\mathbf{v}}+\delta_{A B} \mathbf{E}^{A} \wedge \tilde{\mathbf{E}}^{B}, \\
d \mathbf{e}^{A}= & -\left(\sigma^{A}{ }_{B}-\epsilon_{2} \sigma^{A+n}{ }_{B}\right) \wedge \mathbf{e}^{B}, \\
d \mathbf{E}^{A}= & \left(-u^{1}+\epsilon_{2} v\right) \wedge \mathbf{E}^{A}-\tilde{\mathbf{u}} \wedge \mathbf{e}^{A}-\left(\sigma_{B}^{A}-\epsilon_{2} \sigma_{B}^{A+n}\right) \wedge \mathbf{E}^{B} \\
& +\left(-\epsilon_{2} S_{B C}^{A}+S_{B C}^{A+n}\right) \tilde{\mathbf{e}}^{B} \wedge \tilde{\mathbf{E}}^{C},
\end{aligned}
$$




$$
\begin{aligned}
d \tilde{\mathbf{u}}= & \left(-u^{1}+\epsilon_{2} v\right) \wedge \tilde{\mathbf{u}}-\delta_{A B} \mathbf{E}^{A} \wedge \tilde{\mathbf{e}}^{B}, \\
d \tilde{\mathbf{v}}= & \tilde{\mathbf{u}} \wedge \mathbf{u}+\tilde{\mathbf{v}} \wedge \mathbf{v}+\delta_{A B} \tilde{\mathbf{E}}^{A} \wedge \mathbf{E}^{B}, \\
d \tilde{\mathbf{e}}^{A}= & -\left(\sigma^{A}{ }_{B}+\epsilon_{2} \sigma^{A+n}{ }_{B}\right) \wedge \tilde{\mathbf{e}}^{B}, \\
d \tilde{\mathbf{E}}^{A}= & \left(-u^{1}-\epsilon_{2} v\right) \wedge \tilde{\mathbf{E}}^{A}-\mathbf{u} \wedge \tilde{\mathbf{e}}^{A}-\left(\sigma_{B}^{A}+\epsilon_{2} \sigma_{B}^{A+n}{ }_{B}\right) \wedge \tilde{\mathbf{E}}^{B} \\
& +\left(-\epsilon_{2} S_{B C}^{A}-S_{B C}^{A+n}\right) \mathbf{e}^{B} \wedge \mathbf{E}^{C} .
\end{aligned}
$$

Proof of theorem 1 (c). We first consider $J_{3}$. A basis of the +1 eigendistribution of $J_{3}^{*}$ is given by $\mathcal{B}^{+}=\left(\tilde{\mathbf{u}}, \mathbf{v}, \tilde{\mathbf{e}}^{A}, \mathbf{E}^{A}\right)$. Each term in the exterior derivative of any element in $\mathcal{B}^{+}$ contains a one-form in the set $\mathcal{B}^{+}$. Therefore the distribution is integrable by Frobenius' theorem. A basis of the -1 eigendistribution of $J_{3}^{*}$ is given by $\mathcal{B}^{-}=\left(\mathbf{u}, \tilde{\mathbf{v}}, \mathbf{e}^{A}, \tilde{\mathbf{E}}^{A}\right)$, and by the same argument it is also an integrable distribution. Therefore the almost-para-complex structure $J_{3}$ is integrable.

Let us now consider $J_{3}^{\prime}$ and $\tilde{J}$. A basis of the +1 eigendistribution of $J_{3}^{\prime *}$ is given by $\mathcal{B}^{\prime+}=\left(\tilde{\mathbf{u}}, \tilde{\mathbf{v}}, \tilde{\mathbf{e}}^{A}, \mathbf{E}^{A}\right)$ and a basis of the -1 eigendistribution by $\mathcal{B}^{\prime-}=\left(\mathbf{u}, \mathbf{v}, \mathbf{e}^{A}, \tilde{\mathbf{E}}^{A}\right)$. By the same argument as above $J_{3}^{\prime}$ is integrable. A basis of the +1 eigendistribution of $\tilde{J}$ is given by $\left(\mathbf{u}, \mathbf{v}, \mathbf{e}^{A}, \mathbf{E}^{A}\right)$ and the -1 eigendistribution by $\left(\tilde{\mathbf{u}}, \tilde{\mathbf{v}}, \tilde{\mathbf{e}}^{A}, \tilde{\mathbf{E}}^{A}\right)$. Therefore $\tilde{J}$ is integrable if and only if the cubic tensor $C$ vanishes.

One may gather together the elements of the real partial co-frame (5.13) into the para-quaternionic vielbein

$$
\mathcal{U}^{\mathcal{A} \mu}=\frac{1}{\sqrt{2}}\left(\begin{array}{cccc}
\mathbf{u} & \mathbf{e}^{A} & -\tilde{\mathbf{v}} & -\tilde{\mathbf{E}}^{A} \\
\mathbf{v} & \mathbf{E}^{A} & \tilde{\mathbf{u}} & \tilde{\mathbf{e}}^{A}
\end{array}\right)
$$

Proposition 6 shows that one may replace the complex unit $i$ and holomorphic coordinates in the formal expression for the spatial $c$-map quaternionic vielbein (5.10) with the paracomplex unit $i_{\epsilon_{1}}$ and para-holomorphic coordinates in order to obtain the above expression for the para-quaternionic vielbein in the Euclidean $c$-map with $\left(\epsilon_{1}, \epsilon_{2}\right)=(1,-1)$.

The three endomorphisms $J_{1}, J_{2}, J_{3}$ defined in (4.26) correspond to the following three 2-by-2 matrices $J_{1}^{H}, J_{2}^{H}, J_{3}^{H}$ :

$$
\begin{aligned}
& J_{\alpha}^{*} \mathcal{U}^{\mathcal{A} \mu}=\left(J_{\alpha}^{H}\right)^{\mathcal{A}}{ }_{\mathcal{B}} \mathcal{U}^{\mathcal{B} \mu}, \\
&\left(J_{\alpha}^{H}\right)= \begin{cases}\left(-i \sigma_{\alpha}\right) & \text { if }\left(\epsilon_{1}, \epsilon_{2}\right)=(-1,-1) \\
\left(\tau_{\alpha}\right) & \text { if }\left(\epsilon_{1}, \epsilon_{2}\right)=(-1,+1) \\
\left(\tau_{3},-\tau_{2},-\tau_{1}\right) & \text { if }\left(\epsilon_{1}, \epsilon_{2}\right)=(+1,+1) \\
\left(-\tau_{2},-\tau_{3},-\tau_{1}\right) & \text { if }\left(\epsilon_{1}, \epsilon_{2}\right)=(+1,-1) .\end{cases}
\end{aligned}
$$

Notice that in the last three cases we could have used the same basis $\left(\tau_{\alpha}\right)$. The reason not do so was to allow for the unified expression $(4.26)$ for $\left(J_{\alpha}\right)$ in terms of the orthonormal basis. 
Proof of theorem 2 (c). In the basis (5.15) the Levi-Civita connection one-form decomposes according to (2.21), where $p, q, t, s$ are given by

$$
\begin{aligned}
p & =\left(\begin{array}{cc}
\frac{1}{2} u^{2}+\epsilon_{2} \frac{1}{2} v & -\hat{u}^{1}+\epsilon_{2} \hat{u}^{2} \\
-\epsilon_{2} \hat{u}^{1}-\hat{u}^{2}-\frac{1}{2} u^{2}-\epsilon_{2} \frac{1}{2} v
\end{array}\right), \\
q & =\left(\begin{array}{cc}
-\frac{3}{2} u^{2}+\epsilon_{2} \frac{1}{2} v & \left(\hat{e}^{C}+\epsilon_{2} \hat{e}^{C+n}\right) \delta_{C B} \\
\epsilon_{2} \hat{e}^{A}-\hat{e}^{A+n} & \sigma^{A}{ }_{B}-\epsilon_{2} \sigma^{A+n}{ }_{B}-\frac{1}{2}\left(u^{2}+\epsilon_{2} v\right) \delta_{B}^{A}
\end{array}\right), \\
t & =\left(\begin{array}{cc}
0 & 0 \\
0\left(-\epsilon_{2} S_{B C}^{A}+S_{B C}^{A+n}\right)\left(\hat{e}^{C}+\epsilon_{2} \hat{e}^{C+n}\right)
\end{array}\right), \\
s & =\left(\begin{array}{cc}
0 & 0 \\
0\left(S_{B C}^{A}+\epsilon_{2} S_{B C}^{A+n}{ }_{B C}\right)\left(\hat{e}^{C}-\epsilon_{2} \hat{e}^{C+n}\right)
\end{array}\right) .
\end{aligned}
$$

The Levi-Civita connection is therefore compatible with the para-quaternionic structure.

\section{6 c-map spaces as fibre bundles with bundle metrics}

In section 4.2 we have described $c$-map spaces in terms of the $U_{\epsilon_{1}}(1)$-principal bundle $P=T M \times \mathbb{R} \rightarrow \bar{N}$ equipped with the degenerate symmetric tensor field $g^{\prime}$, see (4.22), which pushes down to the $\varepsilon$-quaternionic Kähler metric $g_{\bar{N}}$. We now turn to a complementary point of view, where $c$-map spaces are locally described as product manifolds

$$
\bar{N}=\bar{M} \times G,
$$

where $\bar{M}$ is the original $\mathrm{PS} \epsilon_{1} \mathrm{~K}$ manifold, which is locally a $\mathrm{PS} \epsilon_{1} \mathrm{~K}$ domain, and where $G$ is the Iwasawa subgroup of $\mathrm{SU}(1, n+2)$. The $\varepsilon$-quaternionic Kähler metric can then be written in the form of a 'bundle metric'

$$
g_{\bar{N}}=\bar{g}+g_{G}(p),
$$

where $\bar{g}$ is the $\mathrm{PS} \epsilon_{1} \mathrm{~K}$ metric, and where $g_{G}(p)$ is a family of left invariant metrics on $G$ which is parametrised by $p \in \bar{M}$. We will show that for fixed $p \in \bar{M}$ the metrics $g_{G}(p)$ are among the symmetric $\epsilon_{1}$-Kähler metrics of constant $\epsilon_{1}$-holomorphic sectional curvature that were discussed in sections 2.1.3 and 2.1.4, and give explicit expressions for the metric, $\epsilon_{1}$-complex structure and $\epsilon_{1}$-Kähler potential.

\subsection{The bundle metric}

We start from (3.3), where we re-write the expression $\left.g_{I J} \partial_{m} X^{I} \partial^{m} \bar{X}^{J}\right|_{D}$ in terms of the physical four-dimensional scalars $z^{A}$. Explicitly, the metric $g_{\bar{N}}$ now takes the form (6.1) where $\bar{g}=\bar{g}_{A B} d z^{A} d \bar{z}^{B}$, see (3.2), and where

$$
\begin{aligned}
g_{G}(p)= & \frac{1}{4} d \phi^{2}-\epsilon_{1} e^{-2 \phi}\left(d \tilde{\phi}+\frac{1}{2}\left(\zeta^{I} d \tilde{\zeta}_{I}-\tilde{\zeta}_{I} d \zeta^{I}\right)\right)^{2} \\
& +\frac{\epsilon_{2}}{2} e^{-\phi}\left(\mathcal{I}_{I J}(p) d \zeta^{I} d \zeta^{J}-\epsilon_{1} \mathcal{I}^{I J}(p)\left(d \tilde{\zeta}_{I}-\mathcal{R}_{I K}(p) d \zeta^{K}\right)\left(d \tilde{\zeta}_{J}-\mathcal{R}_{J L}(p) d \zeta^{L}\right)\right)
\end{aligned}
$$


which as indicated depends on $p \in \bar{M}$. Taking $\bar{M}$ to be a $\mathrm{PS} \epsilon_{1} \mathrm{~K}$ domain, we find that $\bar{N}$ is a product $\bar{N}=\bar{M} \times L \rightarrow \bar{M}$ with fibre $L=\mathbb{R}^{2 n+4}$. The fields $z^{A}$ provide holomorphic coordinates on $\bar{M}$ and $\left(\zeta^{I}, \tilde{\zeta}_{I}, \tilde{\phi}, \phi\right)$ are real coordinates on $L$.

For $\epsilon_{1}=\epsilon_{2}=-1$, the metric (6.2) agrees with the expression in [45] upon making the following field redefinitions

$$
\begin{aligned}
\zeta^{\prime I} & =\frac{1}{\sqrt{2}} \zeta^{I}, \quad \tilde{\zeta}_{I}^{\prime}=\frac{1}{\sqrt{2}} \tilde{\zeta}_{I}, \quad \tilde{\phi}^{\prime}=\tilde{\phi}, \quad \phi^{\prime}=\frac{1}{2} e^{\phi}, \\
\mathcal{I}_{I J}^{\prime} & =-\mathcal{I}_{I J}, \quad \mathcal{R}_{I J}^{\prime}=-\mathcal{R}_{I J},
\end{aligned}
$$

where the 'primed' coordinates are those used in [45].

Following [45] we define the following one-forms:

$$
\begin{aligned}
\eta^{I} & =\sqrt{2} e^{-\phi / 2} d \zeta^{I}, \quad \xi_{I}=\sqrt{2} e^{-\phi / 2}\left(d \tilde{\zeta}_{I}-\mathcal{R}_{I K} d \zeta^{K}\right), \\
\eta^{n+1} & =2 e^{-\phi}\left(d \tilde{\phi}+\frac{1}{2}\left(\zeta^{I} d \tilde{\zeta}_{I}-\tilde{\zeta}_{I} d \zeta^{I}\right)\right), \quad \xi_{n+1}=d \phi,
\end{aligned}
$$

where $I=0, \ldots, n$. n this co-frame the fibre metric is

$$
4 g_{G}=\left(\xi_{n+1}\right)^{2}-\epsilon_{1}\left(\eta^{n+1}\right)^{2}+\epsilon_{2} \mathcal{I}_{I J} \eta^{I} \eta^{J}+\epsilon \mathcal{I}^{I J} \xi_{I} \xi_{J} .
$$

where $\epsilon:=-\epsilon_{1} \epsilon_{2}$. Since $\mathcal{I}_{I J}$ is symmetric and invertible, by a linear change of coordinates, we assume

$$
\mathcal{I}_{I J}=-\eta_{I J},
$$

where $\left(\eta_{I J}\right)=\operatorname{diag}\left(-\epsilon_{1}, 1, \ldots, 1\right)$. Here we used the information about the signature of the matrix $\left(\mathcal{I}_{I J}\right)$ provided in section 3.1.

Thus pointwise with respect to $p \in \bar{M}$ we can bring the fibre metric to the standard form

$$
4 g_{G}=\xi_{n+1}^{2}-\epsilon_{1}\left(\eta^{n+1}\right)^{2}-\epsilon_{2} \eta_{I J} \eta^{I} \eta^{J}-\epsilon \eta^{I J} \xi_{I} \xi_{J} .
$$

The one-forms are invariant under the following group of affine transformations depending on $2 n+4$ real parameters $\left(v^{I}, \tilde{v}_{I}, \alpha, \lambda\right)$ :

$$
\begin{aligned}
\zeta^{I} & \rightarrow e^{\lambda / 2} \zeta^{I}+v^{I}, \\
\tilde{\zeta}_{I} & \rightarrow e^{\lambda / 2} \tilde{\zeta}_{I}+\tilde{v}_{I}, \\
\tilde{\phi} & \rightarrow e^{\lambda} \tilde{\phi}+\frac{1}{2} e^{\lambda / 2}\left(\tilde{v}^{T} \zeta-v^{T} \tilde{\zeta}\right)+\alpha, \\
\phi & \rightarrow \phi+\lambda .
\end{aligned}
$$

The Lie group structure underlying the above affine transformations is

$$
\begin{aligned}
& (v, \tilde{v}, \alpha, \lambda) \cdot\left(v^{\prime}, \tilde{v}^{\prime}, \alpha^{\prime}, \lambda^{\prime}\right)= \\
& \quad\left(v+e^{\lambda / 2} v^{\prime}, \tilde{v}+e^{\lambda / 2} \tilde{v}^{\prime}, \alpha+e^{\lambda} \alpha^{\prime}+\frac{1}{2} e^{\lambda / 2}\left(\tilde{v}^{T} v^{\prime}-v^{T} \tilde{v}^{\prime}\right), \lambda+\lambda^{\prime}\right) .
\end{aligned}
$$


Thus $\mathbb{R}^{2 n+4}$, considered as a Lie group $G$ with the above multiplication, acts on $L=\mathbb{R}^{2 n+4}$ by the affine transformations (6.5). Using this group action we can identify the Lie group $G$ with the $G$-orbit of the point $(0,0,0,0)$, which is all of $L$. The affine transformation (6.5) is then given by the left action of $G$ on itself.

The differentials of the one-forms $\left(\theta^{a}\right)=\left(\eta^{I}, \xi_{I}, \eta^{n+1}, \xi_{n+1}\right)$ are linear combinations of wedge products of the $\theta^{a}$ with constant coefficients:

$$
d \eta^{I}=-\frac{1}{2} \xi_{n+1} \wedge \eta^{I}, \quad d \xi_{I}=-\frac{1}{2} \xi_{n+1} \wedge \xi_{I}, \quad d \eta^{n+1}=-\sum_{A=0}^{n+1} \xi_{A} \wedge \eta^{A}, \quad d \xi_{n+1}=0 .
$$

These coefficients are, in fact, the structure constants of the Lie algebra $\mathfrak{g}$ of the group $G$. This is clear since the forms $\left(\theta^{a}\right)$ can be considered as a basis of the space of left-invariant forms on the group $G$.

The left-invariant vector fields $\left(V_{a}\right)=\left(X_{I}, Y^{I}, Z_{0}, D\right)$ dual to the one-forms $\left(\theta^{a}\right)=$ $\left(\eta^{I}, \xi_{I}, \eta^{n+1}, \xi_{n+1}\right)$ are given explicitly by

$$
\begin{aligned}
& X_{I}=\frac{1}{\sqrt{2}} e^{\phi / 2}\left(\frac{\partial}{\partial \zeta^{I}}+\frac{1}{2} \tilde{\zeta}_{I} \frac{\partial}{\partial \tilde{\phi}}\right), Y^{I}=\frac{1}{\sqrt{2}} e^{\phi / 2}\left(\frac{\partial}{\partial \tilde{\zeta}_{I}}-\frac{1}{2} \zeta^{I} \frac{\partial}{\partial \tilde{\phi}}\right), \\
& Z_{0}=\frac{1}{2} e^{\phi} \frac{\partial}{\partial \tilde{\phi}}, \quad D=\frac{\partial}{\partial \phi} .
\end{aligned}
$$

The non-trivial commutators between these vector fields are

$$
\left[Y^{I}, X_{J}\right]=\delta_{J}^{I} Z_{0}, \quad\left[D, Y^{I}\right]=\frac{1}{2} Y^{I}, \quad\left[D, X_{I}\right]=\frac{1}{2} X_{I}, \quad\left[D, Z_{0}\right]=Z_{0} .
$$

This is a solvable Lie algebra, and looking back at section 2.1.4 we recognise it as the Iwasawa Lie algebra $\mathfrak{g}$ of $\mathrm{SU}(1, n+2)$. Therefore (6.6) is the group multiplication of the Iwasawa group $G$. Thus $g_{G}(p)$ is a family of left-invariant metrics on the fibres $L \simeq G$ of the product $\bar{N}=\bar{M} \times G \rightarrow \bar{M}$.

We saw in section 2.1.4 that the natural $\varepsilon$-complex structure $J_{G}$ (setting $\varepsilon=\epsilon_{1}$ ) on $\mathfrak{g}$ is given by its action on the basis of vector fields $Z_{0}, D, X_{I}, Y^{I}$ via

$$
J_{G} D=-Z_{0}, \quad J_{G} Z_{0}=-\epsilon_{1} D, \quad J_{G} Y^{I}=-\tilde{\eta}^{I J} X_{J}, \quad J_{G} X_{I}=-\epsilon_{1} \tilde{\eta}_{I J} Y^{J}
$$

where $\tilde{\eta}^{I J}=\left\langle Y^{I}, Y^{J}\right\rangle$. Identifying $4 g_{G}$ as given in (6.4) with the scalar product considered in section 2.1.4 we get $\tilde{\eta}_{I J}=\epsilon_{1} \epsilon_{2} \eta_{I J}$. Comparing this with (4.30) we see that the almost $\epsilon_{1}$-complex structure $\tilde{J}$ on $\bar{N}$ obtained by projecting the tensor field $\tilde{J}$ from $P$ to $\bar{N}$ can be written as $^{11}$

$$
\tilde{J}=-\epsilon_{2} J_{\bar{M}}-J_{G} \cdot
$$

In particular, this shows that $J_{G}$ is different from the restriction of the structures $\pm J_{3}$ and $\pm J_{3}^{\prime}$ to the fibres of the projection $\bar{N}=\bar{M} \times G \rightarrow \bar{M}$, with the exception of the case when $\bar{M}$ is a point and therefore $G$ is 4 -dimensional. In the latter case $J_{3}^{\prime}$ coincides with $-J_{G}$, see the end of the next section for a discussion of this special case.

\footnotetext{
${ }^{11}$ Note that $J_{G}=D \wedge Z_{0}+Y^{I} \wedge X_{I}$, when evaluating the endomorphism $J_{G}$ using the scalar product $\langle\cdot, \cdot\rangle$. In (4.30) the metric $g^{\prime}$ is used instead, which restricts to $\frac{1}{4}\langle\cdot, \cdot\rangle$ on the fibre. This leads to an additional factor 4 in (4.30).
} 


\subsection{Kähler potentials for the fibre metrics}

We will now identify the $\varepsilon$-Kähler potentials for the metrics on the fibres $G \simeq L$ of $c$-map spaces, and thus show that they are among the $\varepsilon$-Kähler metrics described in section 2.1.3, where now $\varepsilon=\epsilon_{1}$. We treat all three cases of the $c$-map simultaneously. Along the fibre the matrix $\mathcal{N}_{I J}=\mathcal{R}_{I J}+i_{\epsilon_{1}} \mathcal{I}_{I J}$ is constant. Let us introduce the $\epsilon_{1}$-complex coordinates $\left(C_{I}, S\right)$ via

$$
C_{I}:=\tilde{\zeta}_{I}-\mathcal{N}_{I J} \zeta^{J}, \quad S:=e^{\phi}-\epsilon\left(2 i_{\epsilon_{1}} \tilde{\phi}-\frac{1}{2} C_{I} \mathcal{I}^{I J} \bar{C}_{J}\right) .
$$

One can show that

$$
\begin{aligned}
\sqrt{2} e^{-\phi / 2} d C_{I} & =\xi_{I}-i_{\epsilon_{1}} \mathcal{I}_{I J} \eta^{J}, \\
e^{-\phi}\left(d S-\epsilon d C_{I} \mathcal{I}^{I J} \bar{C}_{J}\right) & =\xi_{n+1}-\epsilon i_{\epsilon_{1}} \eta^{n+1},
\end{aligned}
$$

where differentials are restricted to the fibre, whilst the Kaluza-Klein scalar $\phi$ can be expressed in terms of the fields $\left(C_{I}, S\right)$ as

$$
2 e^{\phi}=S+\bar{S}-\epsilon C_{I} \mathcal{I}^{I J} \bar{C}_{J} .
$$

Hence, the metric on $G$ is given by

$$
g_{G}=\frac{\left|d S-\epsilon d C_{I} \mathcal{I}^{I J} \bar{C}_{J}\right|^{2}}{\left(S+\bar{S}-\epsilon C_{I} \mathcal{I}^{I J} \bar{C}_{J}\right)^{2}}+\epsilon \frac{d C_{I} \mathcal{I}^{I J} d \bar{C}_{J}}{S+\bar{S}-\epsilon C_{I} \mathcal{I}^{I J} \bar{C}_{J}} .
$$

In order to compare to the parametrisation used in section 2.1.3, we introduce the $\epsilon_{1}$-complex variables $\left(u, u^{I}\right)$ via

$$
2 S=\frac{1-u}{1+u}, \quad C_{I}=i_{\epsilon_{1}} \frac{\mathcal{I}_{I J} u^{J}}{1+u},
$$

in terms of which the metric on $G$ becomes

$$
g_{G}=\frac{\left|\bar{u} d u+\epsilon_{2} \bar{u}^{I} \mathcal{I}_{I J} d u^{J}\right|^{2}}{\left(1-|u|^{2}-\epsilon_{2} u^{I} \mathcal{I}_{I J} \bar{u}^{J}\right)^{2}}+\frac{|d u|^{2}+\epsilon_{2} d u^{I} \mathcal{I}_{I J} d \bar{u}^{J}}{1-|u|^{2}-\epsilon_{2} u^{I} \mathcal{I}_{I J} \bar{u}^{J}},
$$

as can be checked by a straightforward but long calculation. A simple calculation shows that the metric $g_{G}$ is $\epsilon_{1}$-Kähler with potential

$$
K=-\log \left(1-|u|^{2}-\epsilon_{2} u^{I} \mathcal{I}_{I J} \bar{u}^{J}\right) .
$$

Since $\mathcal{I}_{I J}$ is constant, non-degenerate and symmetric, we can make a linear coordinate transformation to make it diagonal with entries \pm 1 . Setting

$$
z^{1}=u, \quad z^{a}=u^{a-1}, \quad a=2, \ldots, N=n+2, \quad \bar{\eta}_{a b}=\operatorname{diag}\left(1, \epsilon_{2} \mathcal{I}_{I J}\right),
$$

we obtain

$$
K_{L}=-\log \left(1-\bar{\eta}_{a b} z^{a} \bar{z}^{b}\right)=-\bar{K}
$$

and

$$
g_{L}=-\bar{g}=\left(1-\bar{\eta}_{a b} z^{a} \bar{z}^{b}\right)^{-2}\left(\left(1-\bar{\eta}_{a b} z^{a} \bar{z}^{b}\right) \bar{\eta}_{a b}+\bar{\eta}_{a c} \bar{z}^{c} \bar{\eta}_{b d} z^{d}\right) d z^{a} d \bar{z}^{b}
$$


These metrics $g_{L}$ with $\epsilon_{1}$-Kähler potential $K_{L}$ are, up to an overall sign, among the $\varepsilon$ Kähler metrics $\bar{g}$ with $\varepsilon$-Kähler potential $\bar{K}$ introduced in section 2.1.3, where now $\varepsilon=\epsilon_{1}$. Since the choice of the initial special $\varepsilon$-Kähler metric on $\bar{M}$ determines the signature of the metric on $G$, only a subset of the metrics considered in section 2.1 .3 can be realised by the $c$-map. In particular the Fubini-Study metric on

$$
\mathbb{C} P^{n+2} \simeq \mathrm{U}(n+3) /(\mathrm{U}(1) \times \mathrm{U}(n+2))
$$

cannot be realised. To obtain the negative of the Fubini-Study metric, we would need to take $\epsilon_{1}=-1$, and $\bar{\eta}_{a b}=-\delta_{a b}$ which gives

$$
K_{L}=-\log \left(1+\delta_{a b} z^{a} \bar{z}^{b}\right), \quad g_{L}=-\frac{\left(1+\delta_{c d} z^{c} \bar{z}^{d}\right) \delta_{a b}-\bar{z}^{a} z^{b}}{\left(1+\delta_{c d} z^{c} \bar{z}^{d}\right)^{2}} d z^{a} d \bar{z}^{b} .
$$

However, since $\operatorname{diag}\left(1, \epsilon_{2} \mathcal{I}_{I J}\right) \neq-\delta_{a b}$ it is not possible to obtain this geometry using the $c$-map, even if we were to allow for four-dimensional vector fields with negative kinetic energy.

We now discuss the geometries realised by the three $c$-maps. In order to interpret the resulting signatures in terms of dimensional reduction, we recall that the coordinates $z^{a}$ encode the followings fields: the Kaluza-Klein scalar $\phi$, the dualised Kaluza-Klein vector $\tilde{\phi}$, the components of the four-dimensional vector fields along the direction we reduce over, $\zeta^{I}$, and the scalars dual to the three-dimensional vector fields, $\tilde{\zeta}_{I}$. The signs in front of the kinetic terms of these fields can be read off from the three-dimensional Lagrangian (3.3). Equivalently, they are determined in terms of the signs in the fourdimensional Lagrangian (3.1) through the following general properties of dimensional reduction and Hodge dualisation: (i) spacelike reduction preserves all signs, while timelike reduction reverses the sign for the Kaluza-Klein vector and for the scalars obtained by reducing vector fields; (ii) dualisation of three-dimensional vector fields preserves the sign in Lorenzian signature and reverses it in Euclidean signature. Now we list the cases which can be realised by the different versions of the $c$-map.

1. If we take $\epsilon_{1}=-1$ and $\bar{\eta}_{a b}=\delta_{a b}$, we obtain

$$
K_{L}=-\log \left(1-\delta_{a b} z^{a} \bar{z}^{b}\right), \quad g_{L}=\frac{\left(1-\delta_{c d} z^{c} \bar{z}^{d}\right) \delta_{a b}+\bar{z}_{a} z_{b}}{\left(1-\delta_{c d} z^{c} \bar{z}^{d}\right)^{2}} d z^{a} d \bar{z}^{b}
$$

where $z_{a}=z^{a}$. This is a positive definite Kähler metric on the complex hyperbolic space

$$
\mathbb{C} H^{n+2}=\mathrm{U}(n+2,1) /(\mathrm{U}(n+2) \times \mathrm{U}(1)),
$$

which has constant holomorphic sectional curvature -1 . It is realised, up to a factor 4 , as the fibre geometry $g_{G}=\frac{1}{4} g_{L}$ of the spatial $c$-map, $\epsilon_{1}=\epsilon_{2}=-1$, which has holomorphic sectional curvature -4 . Here we assume that we start with a fourdimensional theory of vector multiplets with positive definite kinetic terms. This implies that $\mathcal{I}_{I J}$ is negative definite. Dimensional reduction over a spacelike direction then results in a three-dimensional theory with positive definite kinetic terms. 
2. Next we take $\epsilon_{1}=-1$ and $\left(\bar{\eta}_{a b}\right)=\operatorname{diag}\left(\mathbb{1}_{\ell},-\mathbb{1}_{k-1}\right)$. Then we obtain

$$
K_{L}=-\log \left(1-\bar{\eta}_{a b} z^{a} \bar{z}^{b}\right), \quad g_{L}=\frac{\left(1-\bar{\eta}_{c d} z^{c} \bar{z}^{d}\right) \bar{\eta}_{a b}+\bar{\eta}_{a c} \bar{z}^{c} \eta_{b d} z^{d}}{\left(1-\bar{\eta}_{c d} z^{c} \bar{z}^{d}\right)^{2}} d z^{a} d \bar{z}^{b}
$$

which are pseudo-Kähler metrics of complex signature $(\ell, k-1)$ with constant holomorphic sectional curvature -1 on the indefinite complex hyperbolic spaces $\mathbb{C} H^{(\ell, k-1)}$. This case is realised by the temporal $c$-map, $\epsilon_{1}=-1, \epsilon_{2}=1$ with the specific value $\ell=1$. Here we use again that $\mathcal{I}_{I J}$ is negative definite, if we start with a theory in Minkowski space-time signature with positive kinetic energy. Upon timelike reduction all scalars resulting from the four-dimensional vector fields have a negative sign, while $\phi$ and $\tilde{\phi}$ have a positive sign, resulting in kinetic terms with signature $(2,2 n+2)$, or complex signature $(1, n+1)$, which corresponds to $\ell=1$. We thus obtain the following indefinite version of the complex hyperbolic space

$$
\mathbb{C} H^{(1, n+1)} \simeq \mathrm{U}(1, n+2) /(\mathrm{U}(1, n+1) \times \mathrm{U}(1)),
$$

with complex signature $(1, n+1)$.

3. Finally we take $\epsilon_{1}=1$ and obtain the same expressions as in (6.9) with complex fields replaced by para-complex fields. This is a para-Kähler metric of constant paraholomorphic sectional curvature -1 on the para-complex hyperbolic space

$$
C H^{n+2} \simeq \mathrm{SL}(n+3) / S(G L(1) \times G L(n+2)) .
$$

The (real) signature is $(n+2, n+2)$ irrespective of the signature of $\bar{\eta}_{a b}$. This geometry is realised as a fibre geometry for the Euclidean $c$-map, $\epsilon_{1}=1, \epsilon_{2}= \pm 1$. The result is independent of the signature of $\mathcal{I}_{I J}$, and hence of $\bar{\eta}_{a b}$, since the metric is paraHermitian and has split-signature. In terms of dimensional reduction, $\phi$ and $\tilde{\phi}$, and $\zeta^{I}$ and $\tilde{\zeta}_{I}$ have opposite signs irrespective of the signs in the four-dimensional Lagrangian. From [3] we know that if we obtain the Euclidean theory by reduction of five-dimensional supergravity with vector multiplets over time, then $\mathcal{I}_{I J}$ has signature $(1, n)$, which reflects the fact that the Kaluza-Klein vector of the $5 \mathrm{~d} / 4 \mathrm{~d}$ reduction has a negative kinetic term.

We remark that by matching the $\varepsilon$-Kähler potentials obtained by the $c$-map to those found in section 2.1.3 we have now proved that the solvable Lie groups presented in section 2.1.4 do indeed provide local realisations of the symmetric spaces discussed in section 2.1.3. We further remark that for the non-compact symmetric spaces of indefinite signature, that is for $\mathbb{C} H^{(l, k-1)}$ and $C H^{n+2}$, the Iwasawa subgroup does not act transitively, though one can find an Iwasawa subgroup which acts with open orbit. In these cases the fibre cannot be identified globally with the corresponding symmetric space, since the fibre has trivial topology, while the symmetric space has non-trivial topology. This is different for $\mathbb{C} H^{n+2}$, where the Iwasawa group acts transitively, so that the fibre is globally isometric to $\mathrm{U}(1, n+2) / \mathrm{U}(1) \times \mathrm{U}(n+2)$. 
The simplest examples of $c$-map spaces are obtained by taking the initial special $\varepsilon$ Kähler manifold to be trivial, $\bar{M}=\{\mathrm{pt}\}$. This corresponds to starting with pure supergravity, and gives rise to a single hypermultiplet, often referred to as the universal hypermultiplet. The corresponding real four-dimensional $\varepsilon$-quaternionic Kähler manifolds ${ }^{12}$ are rather special as they only consist of the fibre, and are therefore locally symmetric spaces which are simultaneously $\varepsilon$-Kähler and $\varepsilon$-quaternionic Kähler. Here the $\varepsilon$-complex structure $J_{G}$ compatible with the $\varepsilon$-Kähler metric coincides with the additional integrable $\varepsilon$-complex structure $-J_{3}^{\prime}$, which is not part of the $\varepsilon$-quaternionic structure. The three different $c$-maps give rise to three different universal hypermultiplets, for which we discuss below the corresponding globally symmetric space.

1. $\epsilon_{1}=-1, \epsilon_{2}=-1$. The symmetric space

$$
\mathbb{C} H^{2}=\mathrm{U}(2,1) /(\mathrm{U}(2) \times \mathrm{U}(1)),
$$

is simultaneously Kähler and quaternionic Kähler. This space is the simplest hypermultiplet geometry occurring in supergravity and appears naturally in various constructions. In particular the classical moduli spaces of M-theory and type-II superstrings on Calabi-Yau threefolds contain this space as a subspace, with the scalar $\phi$ being related to the Calabi-Yau volume and the type-II dilaton, respectively.

2. $\epsilon_{1}=-1, \epsilon_{2}=1$. The symmetric space

$$
\mathbb{C} H^{(1,1)} \simeq \mathrm{U}(2,1) /(\mathrm{U}(1,1) \times \mathrm{U}(1))
$$

is simultaneously pseudo-Kähler and para-quaternionic Kähler. It occurs in the timelike reduction of pure four-dimensional $\mathcal{N}=2$ supergravity [61].

3. $\epsilon_{1}=1, \epsilon_{2}=-1$. The symmetric space

$$
C H^{2} \simeq \mathrm{SL}(3) / S(G L(1) \times G L(2)),
$$

is simultaneously para-Kähler and para-quaternionic Kähler. In [70] it was observed that this geometry is realised by reduction of pure Euclidean supergravity [71], and by dualising the double-tensor multiplet in Euclidean signature.

\section{Acknowledgments}

The work of T.M. is supported in part by the STFC consolidated grant ST/L000431/1. He thanks the Department of Mathematics of the University of Hamburg and the SFB 676 for hospitality and support during several stages of this work. The work of V.C. and O.V. is supported by the German Science Foundation (DFG) under the Collaborative Research Center (SFB) 676 "Particles, Strings and the Early Universe." The work of P.D. is supported by National Research Foundation of Korea grants 2005-0093843, 2010-220C00003 and 2012K2A1A9055280. We would like to thank Malte Dyckmanns for useful discussions.

\footnotetext{
${ }^{12}$ The definition we gave for $\varepsilon$-quaternionic Kähler spaces is only valid if the real dimension is larger than four. As is well known, for the quaternionic case, in four dimensions this definition is not satisfactory, as it only implies orientability. One then takes as a definition that in addition the manifold is Einstein and that the curvature tensor is invariant under the quaternionic structure, a property that in higher dimensions follows from the definition in terms of holonomy.
} 
Open Access. This article is distributed under the terms of the Creative Commons Attribution License (CC-BY 4.0), which permits any use, distribution and reproduction in any medium, provided the original author(s) and source are credited.

\section{References}

[1] V. Cortés, C. Mayer, T. Mohaupt and F. Saueressig, Special geometry of Euclidean supersymmetry. 1. Vector multiplets, JHEP 03 (2004) 028 [hep-th/0312001] [INSPIRE].

[2] V. Cortés, C. Mayer, T. Mohaupt and F. Saueressig, Special geometry of euclidean supersymmetry. II. Hypermultiplets and the c-map, JHEP 06 (2005) 025 [hep-th/0503094] [INSPIRE].

[3] V. Cortés and T. Mohaupt, Special Geometry of Euclidean Supersymmetry III: The Local r-map, instantons and black holes, JHEP 07 (2009) 066 [arXiv:0905.2844] [INSPIRE].

[4] G. Sierra and P.K. Townsend, An introduction to $N=2$ rigid supersymmetry, Preprint LPTENS 83/26 (1983), lectures given at The 19th Karpacz Winter School on Theoretical Physics, Karpacz, Poland, 14-28 February 1983.

[5] S.J. Gates Jr., Superspace Formulation of New Nonlinear $\sigma$-models, Nucl. Phys. B 238 (1984) 349 [inSPIRE].

[6] B. de Wit and A. Van Proeyen, Potentials and Symmetries of General Gauged N=2 Supergravity: Yang-Mills Models, Nucl. Phys. B 245 (1984) 89 [INSPIRE].

[7] A. Strominger, Special geometry, Commun. Math. Phys. 133 (1990) 163 [inSPIRE].

[8] L. Castellani, R. D'Auria and S. Ferrara, Special geometry without special coordinates, Class. Quant. Grav. 7 (1990) 1767 [INSPIRE].

[9] R. D'Auria, S. Ferrara and P. Fré, Special and quaternionic isometries: General couplings in $N=2$ supergravity and the scalar potential, Nucl. Phys. B 359 (1991) 705 [INSPIRE].

[10] B. Craps, F. Roose, W. Troost and A. Van Proeyen, The Definitions of special geometry, hep-th/9606073 [INSPIRE].

[11] L. Andrianopoli et al., $N=2$ supergravity and $N=2$ super Yang-Mills theory on general scalar manifolds: Symplectic covariance, gaugings and the momentum map, J. Geom. Phys. 23 (1997) 111 [hep-th/9605032] [INSPIRE].

[12] D.S. Freed, Special Kähler manifolds, Commun. Math. Phys. 203 (1999) 31 [hep-th/9712042] [INSPIRE].

[13] D.V. Alekseevsky, V. Cortés and C. Devchand, Special complex manifolds, J. Geom. Phys. 42 (2002) 85 [math/9910091] [INSPIRE].

[14] L. Álvarez-Gaumé and D.Z. Freedman, Geometrical Structure and Ultraviolet Finiteness in the Supersymmetric $\sigma$-model, Commun. Math. Phys. 80 (1981) 443 [INSPIRE].

[15] J. Bagger and E. Witten, Matter Couplings in $N=2$ Supergravity, Nucl. Phys. B 222 (1983) 1 [inSPIRE].

[16] J. De Jaegher, B. de Wit, B. Kleijn and S. Vandoren, Special geometry in hypermultiplets, Nucl. Phys. B 514 (1998) 553 [hep-th/9707262] [INSPIRE].

[17] M. Günaydin, G. Sierra and P.K. Townsend, The Geometry of $N=2$ Maxwell-Einstein Supergravity and Jordan Algebras, Nucl. Phys. B 242 (1984) 244 [INSPIRE]. 
[18] D.V. Alekseevsky, V. Cortés, C. Devchand and A. Van Proeyen, Flows on quaternionic Kähler and very special real manifolds, Commun. Math. Phys. 238 (2003) 525 [hep-th/0109094] [INSPIRE].

[19] V. Cortés, Special Kähler manifolds: A Survey, math/0112114 [INSPIRE].

[20] T. Mohaupt and O. Vaughan, Developments in special geometry, J. Phys. Conf. Ser. 343 (2012) 012078 [arXiv:1112.2873] [InSPIRE].

[21] D.Z. Freedman and A. Van Proeyen, Supergravity, Cambridge University Press, (2012).

[22] B. de Wit and A. Van Proeyen, Special geometry, cubic polynomials and homogeneous quaternionic spaces, Commun. Math. Phys. 149 (1992) 307 [hep-th/9112027] [INSPIRE].

[23] D.V. Alekseevsky and V. Cortés, Geometric construction of the r-map: from affine special real to special Kähler manifolds, Comm. Math. Phys. 291 (2009) 579 [arXiv:0811.1658] [INSPIRE].

[24] S. Cecotti, S. Ferrara and L. Girardello, Geometry of Type II Superstrings and the Moduli of Superconformal Field Theories, Int. J. Mod. Phys. A 4 (1989) 2475 [inSPIRE].

[25] S. Ferrara and S. Sabharwal, Quaternionic Manifolds for Type II Superstring Vacua of Calabi-Yau Spaces, Nucl. Phys. B 332 (1990) 317 [INSPIRE].

[26] G.W. Gibbons, M.B. Green and M.J. Perry, Instantons and seven-branes in type IIB superstring theory, Phys. Lett. B 370 (1996) 37 [hep-th/9511080] [INSPIRE].

[27] W. Sabra and O. Vaughan, 10D to 4D Euclidean Supergravity over a Calabi-Yau three-fold, arXiv: 1503.05095 [INSPIRE].

[28] P. Libermann, Sur les structures presque paracomplexes, C.R. Acad. Sci. Paris Sér I Math. 234 (1952) 2517.

[29] P. Libermann, Sur le problème d'équivalence de certaine structures infinitésimales, Ann. Mat. Pura Appl. 36 (1954) 27.

[30] V. Cruceanu, P. Fortuny and P.M. Gadea, A survey of paracomplex geometry, Rocky Mountain J. Math. 26 (1996) 83.

[31] M. Roček, C. Vafa and S. Vandoren, Hypermultiplets and topological strings, JHEP 02 (2006) 062 [hep-th/0512206] [INSPIRE].

[32] B. de Wit and F. Saueressig, Off-shell $N=2$ tensor supermultiplets, JHEP 09 (2006) 062 [hep-th/0606148] [INSPIRE].

[33] D.V. Alekseevsky, V. Cortés and T. Mohaupt, Conification of Kähler and hyper-Kähler manifolds, Commun. Math. Phys. 324 (2013) 637 [arXiv:1205.2964] [INSPIRE].

[34] A. Haydys, Hyper-Kähler and quaternionic Kähler manifolds with $S^{1}$-symmetries, J. Geom. Phys. 58 (2008) 293 [arXiv:0706.4473].

[35] S. Alexandrov, D. Persson and B. Pioline, Wall-crossing, Rogers dilogarithm and the QK/HK correspondence, JHEP 12 (2011) 027 [arXiv:1110.0466] [INSPIRE].

[36] N. Hitchin, On the Hyperkähler/Quaternion Kähler Correspondence, Commun. Math. Phys. 324 (2013) 77 [InSPIRE].

[37] N. Hitchin, The hyperholomorphic line bundle, Algebraic and Complex Geometry, A. Frühbis-Krüger et al. eds., Springer Proc. Math. Stat. 71 (2014) 209 [arXiv:1306.4241]. 
[38] O. Macia and A. Swann, Twist geometry of the c-map, Commun. Math. Phys. 336 (2015) 1329 [arXiv: 1404.0785] [INSPIRE].

[39] O. Macia and A. Swann, Elementary deformations and the hyperKähler-quaternionic Kähler correspondence, arXiv:1404.1169 [INSPIRE].

[40] D. Robles-Llana, F. Saueressig and S. Vandoren, String loop corrected hypermultiplet moduli spaces, JHEP 03 (2006) 081 [hep-th/0602164] [INSPIRE].

[41] D.V. Alekseevsky, V. Cortés, M. Dyckmanns and T. Mohaupt, Quaternionic Kähler metrics associated with special Kähler manifolds, J. Geom. Phys. 92 (2015) 271 [arXiv:1305.3549] [INSPIRE].

[42] N. Hitchin, Quaternionic Kähler moduli spaces, Prog. Math. 271 (2008) 49, K. Galicki and S.R. Simanca eds., Birkhäuser.

[43] M. Günaydin, A. Neitzke, O. Pavlyk and B. Pioline, Quasi-conformal actions, quaternionic discrete series and twistors: $\mathrm{SU}(2,1)$ and $G_{2}(2)$, Commun. Math. Phys. 283 (2008) 169 [arXiv:0707.1669] [INSPIRE].

[44] D. Alekseevsky and V. Cortés, The twistor spaces of a para-quaternionic Kähler manifold, Osaka J. Math. 45 (2008) 215.

[45] V. Cortés, T. Mohaupt and H. Xu, Completeness in supergravity constructions, Commun. Math. Phys. 311 (2012) 191 [arXiv:1101.5103] [InSPIRE].

[46] V. Cortés, M. Dyckmanns and D. Lindemann, Classification of complete projective special real surfaces, arXiv: 1302.4570 [INSPIRE].

[47] V. Cortés, M. Nardmann and S. Suhr, Completeness of hyperbolic centroaffine hypersurfaces, to appear in Comm. Anal. Geom. (2014), arXiv:1407.3251.

[48] T. Mohaupt and O. Vaughan, The Hesse potential, the c-map and black hole solutions, JHEP 07 (2012) 163 [arXiv:1112.2876] [INSPIRE].

[49] D. Errington, T. Mohaupt and O. Vaughan, Non-extremal black hole solutions from the c-map, JHEP 05 (2015) 052 [arXiv: 1408.0923] [INSPIRE].

[50] P. Dempster, D. Errington and T. Mohaupt, Nernst branes from special geometry, JHEP 05 (2015) 079 [arXiv: 1501.07863] [INSPIRE].

[51] N.J. Hitchin, The Moduli space of complex Lagrangian submanifolds, Asian J. Math. 3 (1999) 77 [math/9901069] [INSPIRE].

[52] O. Baues and V. Cortés, Realisation of special Kähler manifolds as parabolic spheres, Proc. Am. Math. Soc. 129 (2001) 2403 [math/9911079] [INSPIRE].

[53] O. Baues and V. Cortés, Proper Affine Hyperspheres which fiber over Projective Special Kähler Manifolds, Asian J. Math. 7 (2003) 115 [math/0205308] [INSPIRE].

[54] V. Cortés, M.-A. Lawn and L. Schäfer, Affine hyperspheres associated to special para-Kähler manifolds, Int. J. Geom. Methods Mod. Phys. 3 (2006) 995.

[55] H. Ooguri, A. Strominger and C. Vafa, Black hole attractors and the topological string, Phys. Rev. D 70 (2004) 106007 [hep-th/0405146] [INSPIRE].

[56] G. Lopes Cardoso, B. de Wit, J. Kappeli and T. Mohaupt, Black hole partition functions and duality, JHEP 03 (2006) 074 [hep-th/0601108] [INSPIRE]. 
[57] G.L. Cardoso, B. de Wit and S. Mahapatra, BPS black holes, the Hesse potential and the topological string, JHEP 06 (2010) 052 [arXiv: 1003.1970] [INSPIRE].

[58] G.L. Cardoso, B. de Wit and S. Mahapatra, Deformations of special geometry: in search of the topological string, JHEP 09 (2014) 096 [arXiv: 1406.5478] [INSPIRE].

[59] S. Ferrara and O. Macia, Real symplectic formulation of local special geometry, Phys. Lett. B 637 (2006) 102 [hep-th/0603111] [INSPIRE].

[60] S. Ferrara and O. Macia, Observations on the Darboux coordinates for rigid special geometry, JHEP 05 (2006) 008 [hep-th/0602262] [INSPIRE].

[61] M. Günaydin, A. Neitzke, B. Pioline and A. Waldron, BPS black holes, quantum attractor flows and automorphic forms, Phys. Rev. D 73 (2006) 084019 [hep-th/0512296] [INSPIRE].

[62] V. Cortés and A. Alekseevsky, Classification of pseudo-Riemannian symmetric spaces of quaternionic Kähler type, Am. Math. Soc. Transl. 213 (2005) 33.

[63] M. Krahe, Para-pluriharmonic maps and twistor spaces, in Handbook of pseudo-Riemannian geometry and supersymmetry, IRMA Lect. Math. Theor. Phys. 16 (2010) 497, European Mathematical Society, Zürich.

[64] V. Cortés, J. Louis, P. Smyth and H. Triendl, On certain Káhler quotients of quaternionic Káhler manifolds, Commun. Math. Phys. 317 (2013) 787 [arXiv:1111.0679] [InSPIRE].

[65] S. Kobayashi and K. Nomitzu, Foundations of Differential Geometry Volume II, Interscience Publishers, (1969).

[66] V. Cortés, A holomorphic representation formula for parabolic hyperspheres, Banach Center Publ. 57 (2002) 11 [math/0107037] [INSPIRE].

[67] B. de Wit, V. Kaplunovsky, J. Louis and D. Lüst, Perturbative couplings of vector multiplets in $N=2$ heterotic string vacua, Nucl. Phys. B 451 (1995) 53 [hep-th/9504006] [INSPIRE].

[68] B. de Wit, $N=2$ electric-magnetic duality in a chiral background, Nucl. Phys. Proc. Suppl. 49 (1996) 191 [hep-th/9602060] [InSPIRE].

[69] M. Krahe, Die c-Abbildung nach Ferrara und Sabharwal: Von spezieller zu quaternionisher Kählergeometrie, Diplomarbeit, Universität Bonn (2001).

[70] U. Theis and S. Vandoren, Instantons in the double tensor multiplet, JHEP 09 (2002) 059 [hep-th/0208145] [INSPIRE].

[71] U. Theis and P. Van Nieuwenhuizen, Ward identities for $N=2$ rigid and local supersymmetry in Euclidean space, Class. Quant. Grav. 18 (2001) 5469 [hep-th/0108204] [INSPIRE]. 